\title{
Cobalt-Catalyzed Diastereo- and Enantioselective Hydrosilylation of Achiral Cyclopropenes
}

\author{
Longyang Dian, Ilan Marek* \\ Schulich Faculty of Chemistry, Technion-Israel Institute of Technology, Haifa, 3200009, \\ Israel \\ * Corresponding Author E-mail: chilanm@technion.ac.il
}

\section{Content}

1. General experimental details .......................................................... 2

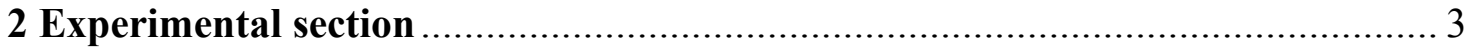

2.1 Ligands used in this project.............................................................. 3

2.2 General Procedure for the Co-catalyzed diastereoselective hydrosilylation of cyclopropenes (Procedure 1) .............................................................. 5

2.3 General Procedure for the Co-catalyzed diastereo- and enantioselective hydrosilylation of cyclopropenes (Procedure 2) ....................................... 9

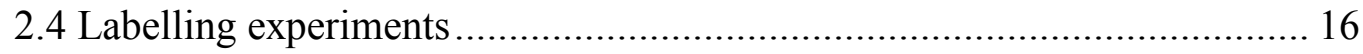

2.5 Confirmation of the absolute configuration ...................................... 17

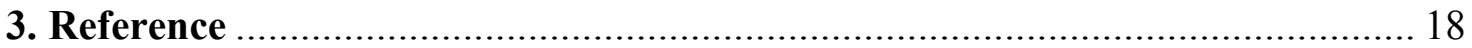

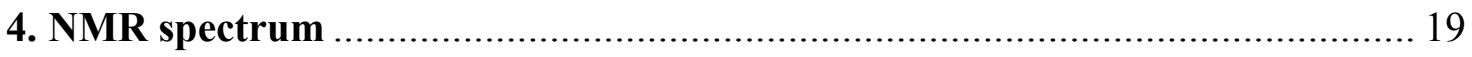




\section{General experimental details}

Unless stated otherwise, reactions were conducted in oven-dried glassware under an atmosphere of argon. Toluene was distilled over sodium/benzophenone prior to use. All other commercially obtained reagents were used as received. Thin-layer chromatography (TLC) was conducted with E. Merck silica gel $60 \mathrm{~F} 254$ precoated plates, $(0.25 \mathrm{~mm})$ and visualized by exposure to UV light (254 $\mathrm{nm}$ ) or stained with anisaldehyde, phosphomolybdic acid or potassium permanganate. Column chromatography was performed using Fluka silica gel $60 \AA$ (40-63mm, 230-400 mesh). ${ }^{1} \mathrm{H}$ NMR spectra were recorded on Bruker spectrometers (400 or 600) and are reported relative to deuterated solvent signals. Chemical shifts are reported in parts per million (ppm) with respect to the residual solvent signal $\mathrm{CDCl}_{3}\left({ }^{1} \mathrm{H}\right.$ NMR: $\delta=7.26 ;{ }^{13} \mathrm{C}$ NMR: $\left.\delta=77.00\right)$. Peak multiplicities are reported as follows: $\mathrm{s}=$ singlet, $\mathrm{bs}=$ broad singlet, $\mathrm{d}=$ doublet, $\mathrm{t}=$ triplet, $\mathrm{dd}=$ doublet of doublets, $\mathrm{td}=$ triplet of doublets, $\mathrm{m}=$ multiplet. app =apparent. High-resolution mass spectra (HRMS) were obtained by the mass spectrometry facility at the Technion. Enantiomeric excesses were determined by HPLC (Agilent $(1) 100$ Series line) using indicated chiral column. Optical rotations were measured on a SCHMIDT and HAENSCHC Unipol L1000 polarimeter with $[\alpha]_{\mathrm{D}}$ values reported in degrees; concentration (c) is in $\mathrm{g} / 100 \mathrm{~mL}$. The cobalt complex $\mathbf{L}_{1} \cdot \mathrm{CoCl}_{2},{ }^{1} \mathbf{L}_{2} \cdot \mathrm{CoCl}_{2}-\mathbf{L}_{5} \cdot \mathrm{CoCl}_{2}{ }^{2}$ cyclopropenes, ${ }^{3}$ aryl silanes ${ }^{4}$ were synthesized according to the known procedure. 


\section{Experimental section}

\subsection{Ligands used in this project}

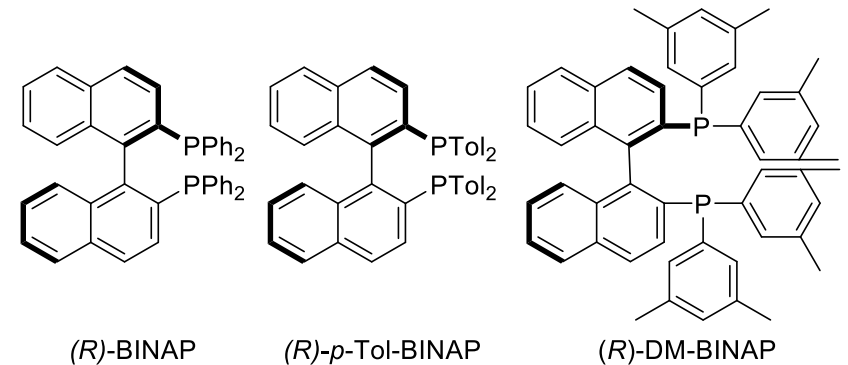

(R)-BINAP

(R)-p-Tol-BINAP

(R)-DM-BINAP<smiles>CC(c1ccccc1)N(C(C)c1ccccc1)P(Oc1ccc2ccccc2c1-c1c(O)ccc2ccccc12)c1ccccc1</smiles>

$(R, S, S)$-phosphoramidite ligand

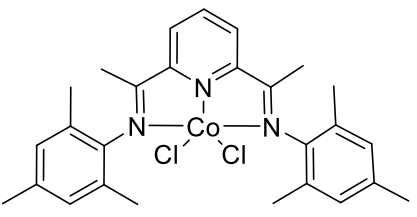
$\mathrm{L}_{1} \cdot \mathrm{CoCl}_{2}$

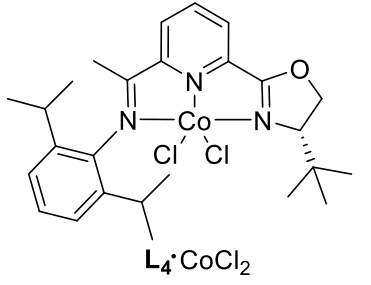<smiles></smiles>

(R)-MonoPhos

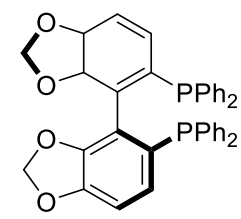

(S)-SEGPHOS<smiles>c1ccc(-c2ccc3c(c2-c2c(P(c4ccccc4)c4ccccc4)ccc4c2CCCC4)CCCC3)cc1</smiles>

(R)- $\mathrm{H}_{8}$-BINAP

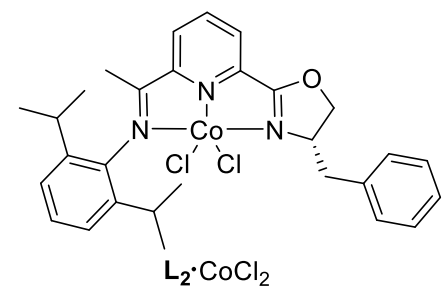

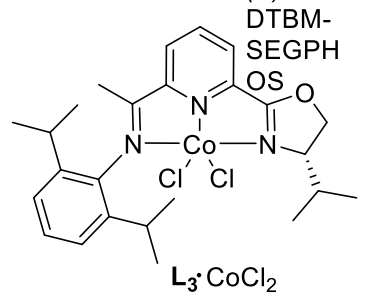

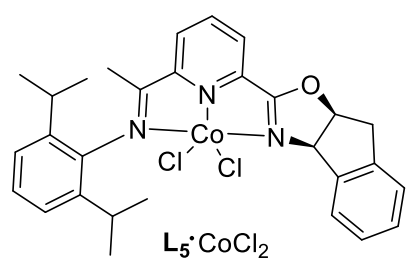




\section{Table S1. Optimization of the Co-catalyzed hydrosilylation of cyclopropene 1a. ${ }^{1}$}

\begin{tabular}{|c|c|c|c|c|c|}
\hline & & $\begin{array}{c}\underset{\text { Ligand }}{\stackrel{\text { Ligand }}{\text { Temp/time }}} \\
\text { Toluene }\end{array}$ & & & \\
\hline Entry & M cat & ligand/additive & $\operatorname{Temp}\left({ }^{\circ} \mathrm{C}\right)$ & $d r^{b}$ & $e r^{c}$ \\
\hline 1 & $\mathrm{Pd}_{2}(\mathrm{dba})_{3}$ & $(R)$-BINAP & 50 & 1 & 1 \\
\hline 2 & $\mathrm{Pd}(\mathrm{OAc})_{2}$ & $(R)$-BINAP & 50 & I & I \\
\hline 3 & $\mathrm{Pd}(\mathrm{acac})_{2}$ & $(R)$-BINAP & 50 & 1 & 1 \\
\hline 4 & $\mathrm{Pd}(\mathrm{TFA})_{2}$ & $(R)$-BINAP & 50 & I & I \\
\hline 5 & $\mathrm{Pd}(\mathrm{dppf}) \mathrm{Cl}_{2}$ & $(R)$-BINAP & 50 & $2: 1$ & I \\
\hline 6 & {$[\mathrm{RhClCOD}]_{2}$} & $(R)$-BINAP & 50 & I & I \\
\hline 7 & {$[\mathrm{RhOMeCOD}]_{2}$} & $(R)$-BINAP & 50 & $3.5: 1$ & I \\
\hline 8 & $\mathrm{Rh}\left(\mathrm{PPh}_{3}\right) \mathrm{Cl}$ & $(R)$-BINAP & 50 & $4.3: 1$ & I \\
\hline 9 & $\mathrm{Rh}(\mathrm{CO})_{2}(\mathrm{acac})$ & $(R)$-BINAP & 50 & $4: 1$ & 1 \\
\hline 10 & $\mathrm{Rh}(\mathrm{OAc})_{2}$ & $(R)$-BINAP & 50 & $2.3: 1$ & 1 \\
\hline 11 & $\mathrm{RhCl}_{3}$ & $(R)$-BINAP & 50 & $8.1: 1$ & $50: 50$ \\
\hline 12 & $\mathrm{RhCl}_{3}$ & $(R)$-TolBINAP & 50 & $19: 1$ & $50: 50$ \\
\hline 13 & $\mathrm{RhCl}_{3}$ & $(R)$-DM-BINAP & 50 & $15.7: 1$ & $50: 50$ \\
\hline 14 & $\mathrm{RhCl}_{3}$ & $(R)-\mathrm{H}_{8}-\mathrm{BINAP}$ & 50 & $10.1: 1$ & $50: 50$ \\
\hline 15 & $\mathrm{RhCl}_{3}$ & $(R, S, S)$-phosphoramidite & 50 & $3.3: 1$ & 1 \\
\hline 16 & $\mathrm{RhCl}_{3}$ & (R)-Monophos & 50 & 1 & I \\
\hline 17 & $\mathrm{RhCl}_{3}$ & (S)-Segphos & 50 & $11.5: 1$ & $50: 50$ \\
\hline 18 & $\mathrm{RhCl}_{3}$ & (S)-DTBM-Segphos & 50 & $4.9: 1$ & $50: 50$ \\
\hline 19 & $\mathrm{~L}_{9} \cdot \mathrm{CoCl}_{2}$ & $\mathrm{NaBHEt}_{3}$ & -15 & $20: 1$ & I \\
\hline 20 & $\mathbf{L}_{10} \cdot \mathrm{CoCl}_{2}$ & $\mathrm{NaBHEt}_{3}$ & -15 & 1 & 1 \\
\hline 21 & $\mathrm{~L}_{11} \cdot \mathrm{CoCl}_{2}$ & $\mathrm{NaBHEt}_{3}$ & -15 & $10: 1$ & $86: 14$ \\
\hline 22 & $\mathbf{L}_{12} \cdot \mathrm{CoCl}_{2}$ & $\mathrm{NaBHEt}_{3}$ & -15 & $20: 1$ & $96: 04$ \\
\hline 23 & $\mathbf{L}_{13} \cdot \mathrm{CoCl}_{2}$ & $\mathrm{NaBHEt}_{3}$ & -15 & 7.3:1 & $96: 04$ \\
\hline $24^{d}$ & $\mathrm{~L}_{12} \cdot \mathrm{CoCl}_{2}$ & $\mathrm{NaBHEt}_{3}$ & -15 & $20: 1$ & $96: 04$ \\
\hline 25 & $\mathrm{~L}_{12} \cdot \mathrm{CoCl}_{2}$ & 1 & -15 & 1 & 1 \\
\hline 26 & I & $\mathrm{NaBHEt}_{3}$ & -15 & 1 & I \\
\hline
\end{tabular}

a The reactions were run on a $0.10 \mathrm{mmol}$ scale, $[\mathrm{Co}](10 \mathrm{~mol} \%)$, ligand or additive $(20 \mathrm{~mol} \%)$ in toluene $(0.1 \mathrm{M})$ and the reaction mixture was stirred at indicated temperature for $5 \mathrm{~h}$. NA, not analyzed; ND, no detection of the desired product. Entry 1-18, 50-70\% conversion of cyclopropene 1a; entry 19, 21-24, full conversion of cyclopropene 1a; entry 20, $10 \%$ conversion, trace 2 a was detected; entry $25-26$, no conversion of cyclopropene 1a. ${ }^{b}$ Determined by GC analysis of hydrolyzed aliquots. ${ }^{c}$ Determined by chiral HPLC. d 5 mol\% cobalt complex $\left(\mathrm{L}_{4} \cdot \mathrm{CoCl}_{2}\right)$ and $10 \mathrm{~mol}_{\%} \mathrm{NaBHEt}_{3}$ were used in the reaction. 


\subsection{General Procedure for the Co-catalyzed diastereoselective hydrosilylation of cyclopropenes (Procedure 1)}

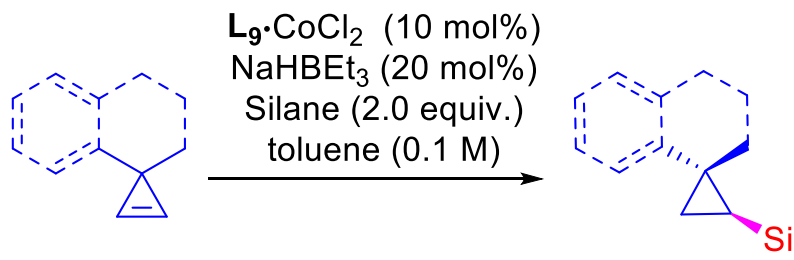

The Cobalt complex $\mathbf{L}_{9} \cdot \mathrm{CoCl}_{2}(10.5 \mathrm{mg}, 0.020 \mathrm{mmol}, 10 \mathrm{~mol} \%)$ was added to the flask under $\mathrm{N}_{2}$ atmosphere charged with a stirring bar and toluene $(2 \mathrm{~mL}, 0.1 \mathrm{M})$. After stirring at room temperature for $10 \mathrm{~min}$, the corresponding arylsilane $(0.40 \mathrm{mmol}, 2.0$ equiv. $)$ was added to the flask, followed by the dropwise addition of the corresponding cyclopropene $(0.20 \mathrm{mmol})$. After cooling down to $-15{ }^{\circ} \mathrm{C}, \mathrm{NaBHEt}_{3}(1.0 \mathrm{M}$ in toluene, $40 \mu \mathrm{L}, 0.040$ mmol, $20 \mathrm{~mol} \%$ ) was added dropwise to the reaction mixture, which was further stirring for about $5 \mathrm{~h}$ until full conversion of cyclopropene (checked by analysis by TLC). The reaction mixture was evaporated directly and the oily residue was purified by flash column to afford the corresponding racemic product.

\section{2-Methyl-2-phenylcyclopropyl)(phenyl)silane (2a) ${ }^{5}$}

Silycyclopropane 2a was prepared according to the general procedure 1. After standard work-up and purification by flash column (100\% Hexane), 2a was obtained as a colorless oil (37.1 $\mathrm{mg}, 78 \%$ yield). ${ }^{1} \mathrm{H}$ NMR $\left(400 \mathrm{MHz}, \mathrm{CDCl}_{3}\right) \delta 7.63-7.55(\mathrm{~m}, 2 \mathrm{H}), 7.32(\mathrm{qd}, 3 \mathrm{H}, J=5.9,3.8 \mathrm{~Hz}), 7.21$ (d, $4 \mathrm{H}, J=4.3 \mathrm{~Hz}), 7.09$ (dt, $1 \mathrm{H}, J=8.6,4.4 \mathrm{~Hz}), 4.42(\mathrm{dd}, 1 \mathrm{H}, J=6.0,3.9 \mathrm{~Hz}), 4.37$ (dd, $1 \mathrm{H}, J=6.0$, $3.8 \mathrm{~Hz}), 1.44$ (s, 3H), 1.33 (dd, 1H, $J=10.3,3.9 \mathrm{~Hz}), 0.82(\mathrm{dd}, 1 \mathrm{H}, J=7.4,3.9 \mathrm{~Hz}), 0.39-0.30$ (m, 1H). HRMS (TOF-MS ES ${ }^{+}$): $[\mathrm{M}+\mathrm{H}]^{+}$, calculated for $\mathrm{C}_{16} \mathrm{H}_{19} \mathrm{Si}: 239.1251$; found 239.1246.

\section{2-(4-Chlorophenyl)-2-methylcyclopropyl)(phenyl)silane (2b) ${ }^{5}$}

Silycyclopropane $\mathbf{2 b}$ was prepared according to the general procedure 1. After standard work-up and purification by flash column (100\% Hexane), $2 \mathbf{b}$ was obtained as a colorless oil $(48.4 \mathrm{mg}, 89 \%$ yield). ${ }^{1} \mathrm{H}$ NMR (100 MHz, $\left.\mathrm{CDCl}_{3}\right) \delta 7.57$ (dd, $\left.1 \mathrm{H}, J=7.5,1.7 \mathrm{~Hz}\right), 7.36-7.27(\mathrm{~m}, 3 \mathrm{H}), 7.19-$ $7.06(\mathrm{~m}, 4 \mathrm{H}), 4.38$ (ddd, $2 \mathrm{H}, J=19.3,6.1,3.8 \mathrm{~Hz}), 1.40$ (s, 3H), 1.28 (dd, $1 \mathrm{H}, J=10.3,4.0 \mathrm{~Hz})$, $0.81(\mathrm{dd}, 1 \mathrm{H}, J=7.5,4.0 \mathrm{~Hz}), 0.29$ (ddt, $1 \mathrm{H}, J=11.1,7.5,3.8 \mathrm{~Hz})$.

\section{2-(4-Methoxyphenyl)-2-methylcyclopropyl)(phenyl)silane (2c) ${ }^{5}$}

Silycyclopropane $2 \mathbf{c}$ was prepared according to the general procedure 1. After standard work-up and purification by flash column (100\% Hexane), $2 \mathbf{c}$ was obtained as a colorless oil $(49.3 \mathrm{mg}, 92 \%$ yield). ${ }^{1} \mathrm{H}$ NMR (400 MHz, $\left.\mathrm{CDCl}_{3}\right) \delta 7.59$ (dd, $\left.2 \mathrm{H}, J=7.4,1.9 \mathrm{~Hz}\right), 7.35-7.27(\mathrm{~m}, 3 \mathrm{H}), 7.16-$ $7.09(\mathrm{~m}, 2 \mathrm{H}), 6.77-6.71(\mathrm{~m}, 2 \mathrm{H}), 4.38(\mathrm{ddd}, 2 \mathrm{H}, J=17.9,6.0,3.9 \mathrm{~Hz}), 3.70(\mathrm{~s}, 3 \mathrm{H}), 1.40(\mathrm{~s}, 3 \mathrm{H})$, $1.26(\mathrm{dd}, 1 \mathrm{H}, J=10.2,3.8 \mathrm{~Hz}), 0.77$ (dd, $1 \mathrm{H}, J=7.4,3.8 \mathrm{~Hz}), 0.28$ (ddd, 1H, $J=14.2,7.5,3.8$ $\mathrm{Hz}$,$) .$

\section{2-Methyl-2-(p-tolyl)cyclopropyl)(phenyl)silane (2d)}


Silycyclopropane $\mathbf{2 d}$ was prepared according to the general procedure 1. After standard work-up and purification by flash column (100\% Hexane), 2d was obtained as a colorless oil (39.3 $\mathrm{mg}, 78 \%$ yield). ${ }^{1} \mathrm{H}$ NMR (400 MHz, $\left.\mathrm{CDCl}_{3}\right) \delta 7.77(\mathrm{dd}, 2 \mathrm{H}, J=7.5,1.9 \mathrm{~Hz}), 7.54-7.45(\mathrm{~m}, 3 \mathrm{H}), 7.28$ (d, $2 \mathrm{H}, J=8.2 \mathrm{~Hz}$ ), 7.19 (d, 2H, $J=7.9 \mathrm{~Hz}), 4.57$ (ddd, $2 \mathrm{H}, J=17.8,6.0,3.8 \mathrm{~Hz}), 2.41(\mathrm{~s}, 3 \mathrm{H}), 1.60$ (s, 3H), 1.47 (dd, $1 \mathrm{H}, J=10.3,3.8 \mathrm{~Hz}), 0.97$ (dd, $1 \mathrm{H}, J=7.4,3.9 \mathrm{~Hz}), 0.49$ (ddd, $1 \mathrm{H}, J=14.2,7.5$, $3.8 \mathrm{~Hz}) .{ }^{13} \mathrm{C} \mathrm{NMR}\left(100 \mathrm{MHz}, \mathrm{CDCl}_{3}\right) \delta 144.9,135.3,132.8,129.6,128.9,128.0,126.7,25.0,23.7$, 20.9, 20.3, 8.4. HRMS (TOF-MS $\mathrm{ES}^{+}$): $[\mathrm{M}+\mathrm{H}]^{+}$, calculated for $\mathrm{C}_{17} \mathrm{H}_{21} \mathrm{Si}$ : 253.1407; found 253.1415 .

\section{2-(4-Ethylphenyl)-2-methylcyclopropyl)(phenyl)silane (2e)}

Silycyclopropane 2e was prepared according to the general procedure 1. After standard work-up and purification by flash column (100\% Hexane), $2 \mathrm{e}$ was obtained as a colorless oil $(41.0 \mathrm{mg}, 77 \%$ yield). ${ }^{1} \mathrm{H}$ NMR (400 MHz, $\mathrm{CDCl}_{3}$ ) $\delta 7.72(\mathrm{dd}, 2 \mathrm{H}, J=7.3,1.8 \mathrm{~Hz}$ ), 7.45 (q, 3H, $J=6.0 \mathrm{~Hz}$ ), 7.26 $(\mathrm{d}, 1 \mathrm{H}, J=8.2 \mathrm{~Hz}), 7.17(\mathrm{~d}, 1 \mathrm{H}, J=8.2 \mathrm{~Hz}), 4.52(\mathrm{ddd}, 1 \mathrm{H}, J=18.2,6.0,3.9 \mathrm{~Hz}), 2.67(\mathrm{q}, 2 \mathrm{H}, J=$ $7.6 \mathrm{~Hz}), 1.56$ (s, 3H), 1.43 (dd, $1 \mathrm{H}, J=10.2,3.8 \mathrm{~Hz}), 1.28$ (t, 3H, $J=7.6 \mathrm{~Hz}), 0.92(\mathrm{dd}, 1 \mathrm{H}, J=$ 7.4, $3.9 \mathrm{~Hz}), 0.45$ (ddd, $1 \mathrm{H}, J=14.3,7.5,3.8 \mathrm{~Hz}) .{ }^{13} \mathrm{C} \mathrm{NMR}\left(100 \mathrm{MHz}, \mathrm{CDCl}_{3}\right) \delta 145.1,141.7$, 135.3, 132.8, 129.6, 128.0, 127.7, 126.7, 28.4, 24.9, 23.6, 20.4, 15.6, 8.5. HRMS (TOF-MS ES ${ }^{+}$): $[\mathrm{M}+\mathrm{H}]^{+}$, calculated for $\mathrm{C}_{18} \mathrm{H}_{23} \mathrm{Si}: 267.1564$; found 267.1566 .

\section{3',4'-dihydro-2'H-spiro[cyclopropane-1,1'-naphthalen]-2-yl)(phenyl)silane (2f) ${ }^{5}$}

Silycyclopropane $\mathbf{2 f}$ was prepared according to the general procedure 1. After standard work-up and purification by flash column (100\% Hexane), $2 \mathbf{f}$ was obtained as a colorless oil $(43.3 \mathrm{mg}, 82 \%$ yield). ${ }^{1} \mathrm{H}$ NMR (400 MHz, $\left.\mathrm{CDCl}_{3}\right) \delta 7.62-7.47(\mathrm{~m}, 2 \mathrm{H}), 7.38-7.24(\mathrm{~m}, 3 \mathrm{H}), 7.07-6.92(\mathrm{~m}$, $3 \mathrm{H}), 6.69(\mathrm{~d}, 1 \mathrm{H}, J=7.6 \mathrm{~Hz}), 4.43(\mathrm{dd}, 1 \mathrm{H}, J=6.2,3.0 \mathrm{~Hz}), 4.32(\mathrm{dd}, 1 \mathrm{H}, J=6.1,4.2 \mathrm{~Hz}), 2.80(\mathrm{t}$, $2 \mathrm{H}, J=6.0 \mathrm{~Hz}), 1.85-1.69(\mathrm{~m}, 4 \mathrm{H}), 1.49(\mathrm{~s}, 3 \mathrm{H}), 1.40(\mathrm{dd}, 1 \mathrm{H}, J=10.3,4.1 \mathrm{~Hz}), 0.90(\mathrm{dd}, 1 \mathrm{H}, J$ $=7.8,4.1 \mathrm{~Hz}), 0.44(\mathrm{ddd}, 1 \mathrm{H}, J=14.3,7.4,3.6 \mathrm{~Hz})$.

\section{(4-Methoxyphenyl)(2-methyl-2-phenylcyclopropyl)silane (2g)}

Silycyclopropane $\mathbf{2 g}$ was prepared according to the general procedure 1. After standard work-up and purification by flash column (100\% Hexane), $\mathbf{2 g}$ was obtained as a colorless oil $(39.1 \mathrm{mg}, 73 \%$ yield). ${ }^{1} \mathrm{H}$ NMR (400 MHz, $\left.\mathrm{CDCl}_{3}\right) \delta 7.50$ (d, $2 \mathrm{H}, J=8.4 \mathrm{~Hz}$ ), 7.20 (t, $4 \mathrm{H}, J=5.1 \mathrm{~Hz}$ ), 7.09 (dd, $1 \mathrm{H}, J=8.6,4.3 \mathrm{~Hz}), 6.86$ (d, 1H, $J=8.4 \mathrm{~Hz}), 4.37$ (ddd, $1 \mathrm{H}, J=17.8,6.0,3.8 \mathrm{~Hz}), 3.74(\mathrm{~s}, 3 \mathrm{H})$, $1.43(\mathrm{~s}, 3 \mathrm{H}), 1.30(\mathrm{dd}, 1 \mathrm{H}, J=10.3,3.8 \mathrm{~Hz}), 0.79(\mathrm{dd}, 1 \mathrm{H}, J=7.4,3.9 \mathrm{~Hz}), 0.35-0.27(\mathrm{~m}, 1 \mathrm{H})$. ${ }^{13} \mathrm{C} \mathrm{NMR}\left(100 \mathrm{MHz}, \mathrm{CDCl}_{3}\right) \delta 160.9,148.0,136.7,128.2,126.8,125.7,123.2,113.9,55.0,25.2$, 23.5, 20.4, 8.9. HRMS (TOF-MS ES ${ }^{+}$): $[\mathrm{M}+\mathrm{H}]^{+}$, calculated for $\mathrm{C}_{17} \mathrm{H}_{21} \mathrm{OSi}$ : 269.1356; found 269.1366 .

\section{2-(4-Chlorophenyl)-2-methylcyclopropyl)(4-methoxyphenyl)silane (2h)}

Silycyclopropane $\mathbf{2 h}$ was prepared according to the general procedure 1. After standard work-up and purification by flash column (100\% Hexane), $\mathbf{2 h}$ was obtained as a colorless oil (42.9 $\mathrm{mg}, 70 \%$ yield). ${ }^{1} \mathrm{H}$ NMR (400 MHz, $\left.\mathrm{CDCl}_{3}\right) \delta 7.54-7.46(\mathrm{~m}, 2 \mathrm{H}), 7.19-7.10(\mathrm{~m}, 5 \mathrm{H}), 6.90-6.82(\mathrm{~m}$, 2H), $4.39(\mathrm{dd}, 1 \mathrm{H}, J=6.1,3.8 \mathrm{~Hz}), 4.34(\mathrm{dd}, 1 \mathrm{H}, J=6.1,3.6 \mathrm{~Hz}), 3.75(\mathrm{~s}, 3 \mathrm{H}), 1.40(\mathrm{~s}, 3 \mathrm{H}), 1.27$ $(\mathrm{dd}, 1 \mathrm{H}, J=10.3,4.0 \mathrm{~Hz}), 0.80(\mathrm{dd}, 1 \mathrm{H}, J=7.5,4.0 \mathrm{~Hz}), 0.26(\mathrm{td}, 1 \mathrm{H}, J=7.2,3.7 \mathrm{~Hz}) .{ }^{13} \mathrm{C}$ NMR 
$\left(100 \mathrm{MHz}, \mathrm{CDCl}_{3}\right) \delta 161.0,146.5,136.7,131.3,128.3,128.2,123.0,113.9,55.1,24.8,23.4,20.4$, 9.1. HRMS (TOF-MS ES ${ }^{+}$): [M+H] $]^{+}$, calculated for $\mathrm{C}_{17} \mathrm{H}_{20} \mathrm{ClOSi}$ : 303.0966; found 303.0971.

(4-Methoxyphenyl)(2-(4-methoxyphenyl)-2-methylcyclopropyl)silane (2i)

Silycyclopropane $2 \mathbf{i}$ was prepared according to the general procedure 1. After standard work-up and purification by flash column (100\% Hexane), $2 \mathbf{i}$ was obtained as a colorless oil (47.7 $\mathrm{mg}, 80 \%$ yield). ${ }^{1} \mathrm{H}$ NMR (400 MHz, $\left.\mathrm{CDCl}_{3}\right) \delta 7.51(\mathrm{~d}, 2 \mathrm{H}, J=8.5 \mathrm{~Hz}), 7.13(\mathrm{~d}, 2 \mathrm{H}, J=8.7 \mathrm{~Hz}), 6.87(\mathrm{~d}$, $2 \mathrm{H}, J=8.5 \mathrm{~Hz}), 6.74(\mathrm{~d}, 2 \mathrm{H}, J=8.7 \mathrm{~Hz}), 4.38(\mathrm{dd}, 1 \mathrm{H}, J=6.1,3.9 \mathrm{~Hz}), 4.34(\mathrm{dd}, 1 \mathrm{H}, J=6.1,3.7$ $\mathrm{Hz}), 3.76(\mathrm{~s}, 3 \mathrm{H}), 3.71(\mathrm{~s}, 3 \mathrm{H}), 1.40(\mathrm{~s}, 3 \mathrm{H}), 1.25(\mathrm{dd}, 1 \mathrm{H}, J=10.3,3.8 \mathrm{~Hz}), 0.76(\mathrm{dd}, 1 \mathrm{H}, J=7.4$, $3.8 \mathrm{~Hz}), 0.26(\mathrm{td}, 1 \mathrm{H}, J=7.1,3.7 \mathrm{~Hz}) .{ }^{13} \mathrm{C} \mathrm{NMR}\left(100 \mathrm{MHz}, \mathrm{CDCl}_{3}\right) \delta 160.9,157.6,140.3,136.7$, 128.0, 123.4, 113.9, 113.6, 55.3, 55.1, 24.8, 23.9, 20.1, 8.4. HRMS (TOF-MS ES ${ }^{+}$): $[\mathrm{M}+\mathrm{H}]^{+}$, calculated for $\mathrm{C}_{18} \mathrm{H}_{23} \mathrm{O}_{2} \mathrm{Si}$ : 299.1462; found 299.1455.

\section{(2-Methyl-2-phenylcyclopropyl)(p-tolyl)silane (2j)}

Silycyclopropane $\mathbf{2} \mathbf{j}$ was prepared according to the general procedure 1 . After standard work-up and purification by flash column (100\% Hexane), $\mathbf{2} \mathbf{j}$ was obtained as a colorless oil (37.8 $\mathrm{mg}, 75 \%$ yield). ${ }^{1} \mathrm{H}$ NMR (400 MHz, $\left.\mathrm{CDCl}_{3}\right) \delta 7.48$ (d, $\left.2 \mathrm{H}, J=7.6 \mathrm{~Hz}\right), 7.20(\mathrm{~d}, 4 \mathrm{H}, J=4.3 \mathrm{~Hz}), 7.13$ (d, $2 \mathrm{H}, J=7.7 \mathrm{~Hz}), 7.11-7.05(\mathrm{~m}, 1 \mathrm{H}), 4.40(\mathrm{dd}, 1 \mathrm{H}, J=6.0,3.8 \mathrm{~Hz}), 4.35$ (dd, $1 \mathrm{H}, J=6.1,3.7 \mathrm{~Hz})$, 2.29 (s, 3H), 1.43 (s, 3H), 1.31 (dd, $1 \mathrm{H}, J=10.3,3.9 \mathrm{~Hz}), 0.80$ (dd, $1 \mathrm{H}, J=7.5,3.9 \mathrm{~Hz}), 0.32$ (ddt, $1 \mathrm{H}, J=11.0,7.5,3.8 \mathrm{~Hz}) .{ }^{13} \mathrm{C} \mathrm{NMR}\left(100 \mathrm{MHz}, \mathrm{CDCl}_{3}\right) \delta 147.9,139.6,135.3,129.4,128.9,128.2$, 126.8, 125.7, 25.2, 23.5, 21.5, 20.4, 8.7. HRMS (TOF-MS ES ${ }^{+}$): $[\mathrm{M}+\mathrm{H}]^{+}$, calculated for $\mathrm{C}_{17} \mathrm{H}_{21} \mathrm{Si}$ : 253.1407; found 253.1409.

\section{(4-Chlorophenyl)(2-methyl-2-phenylcyclopropyl)silane (2k)}

Silycyclopropane $\mathbf{2 k}$ was prepared according to the general procedure 1. After standard work-up and purification by flash column (100\% Hexane), $2 \mathbf{k}$ was obtained as a colorless oil (36.4 $\mathrm{mg}, 67 \%$ yield). ${ }^{1} \mathrm{H}$ NMR $\left(400 \mathrm{MHz}, \mathrm{CDCl}_{3}\right) \delta 7.51(\mathrm{~d}, 2 \mathrm{H}, J=8.1 \mathrm{~Hz}), 7.30(\mathrm{~d}, 2 \mathrm{H}, J=8.1 \mathrm{~Hz}), 7.23-7.17$ $(\mathrm{m}, 4 \mathrm{H}), 7.10(\mathrm{td}, 1 \mathrm{H}, J=6.0,2.7 \mathrm{~Hz}), 4.40(\mathrm{dd}, 1 \mathrm{H}, J=6.1,3.9 \mathrm{~Hz}), 4.35(\mathrm{dd}, 1 \mathrm{H}, J=6.1,3.7$ $\mathrm{Hz}), 1.43$ (s, 3H), 1.34 (dd, $1 \mathrm{H}, J=10.3,4.0 \mathrm{~Hz}), 0.81$ (dd, $1 \mathrm{H}, J=7.5,3.9 \mathrm{~Hz}), 0.31$ (ddt, $1 \mathrm{H}, J=$ 11.0, 7.5, 3.8 Hz). ${ }^{13} \mathrm{C}$ NMR $\left(100 \mathrm{MHz}, \mathrm{CDCl}_{3}\right) \delta 147.6,136.6,136.1,131.0,128.3,128.3,126.8$, 125.8, 25.4, 23.6, 20.4, 8.4. HRMS (TOF-MS ES ${ }^{+}$): $[\mathrm{M}+\mathrm{H}]^{+}$, calculated for $\mathrm{C}_{16} \mathrm{H}_{18} \mathrm{ClSi}$ : 273.0861; found 273.0869 .

\section{(2-Methyl-2-phenylcyclopropyl)(o-tolyl)silane (2l)}

Silycyclopropane $\mathbf{2 l}$ was prepared according to the general procedure 1. After standard work-up and purification by flash column (100\% Hexane), $2 \mathbf{l}$ was obtained as a colorless oil $(29.2 \mathrm{mg}, 58 \%$ yield). ${ }^{1} \mathrm{H}$ NMR $\left(400 \mathrm{MHz}, \mathrm{CDCl}_{3}\right) \delta 7.56(\mathrm{~d}, 1 \mathrm{H}, J=7.3 \mathrm{~Hz}), 7.29-7.19(\mathrm{~m}, 6 \mathrm{H}), 7.15-7.08(\mathrm{~m}$, $3 \mathrm{H}), 4.45(\mathrm{dd}, 1 \mathrm{H}, J=5.5,3.5 \mathrm{~Hz}), 4.40-4.37(\mathrm{~m}, 1 \mathrm{H}), 2.43(\mathrm{~s}, 3 \mathrm{H}), 1.44(\mathrm{~s}, 3 \mathrm{H}), 1.34(\mathrm{dd}, 1 \mathrm{H}, J$ $=10.3,3.8 \mathrm{~Hz}), 0.79(\mathrm{dd}, 1 \mathrm{H}, J=7.5,3.8 \mathrm{~Hz}), 0.34(\mathrm{ddd}, 1 \mathrm{H}, J=14.2,7.4,3.7 \mathrm{~Hz}) .{ }^{13} \mathrm{C}$ NMR $(100$ $\left.\mathrm{MHz}, \mathrm{CDCl}_{3}\right) \delta 147.9,144.1,136.3,132.1,130.1,129.3,128.3,126.6,125.7,125.1,25.3,23.2$, 22.6, 20.9, 8.4. HRMS (TOF-MS $\mathrm{ES}^{+}$): $[\mathrm{M}+\mathrm{H}]^{+}$, calculated for $\mathrm{C}_{17} \mathrm{H}_{21} \mathrm{Si}$ : 253.1407; found 253.1412 .

\section{(2-Methoxyphenyl)(2-methyl-2-phenylcyclopropyl)silane (2m)}


Silycyclopropane $\mathbf{2 m}$ was prepared according to the general procedure 1. After standard work-up and purification by flash column (100\% Hexane), $\mathbf{2 m}$ was obtained as a colorless oil $(40.2 \mathrm{mg}, 75 \%$ yield). ${ }^{1} \mathrm{H}$ NMR $\left(400 \mathrm{MHz}, \mathrm{CDCl}_{3}\right) \delta 7.48(\mathrm{dd}, 1 \mathrm{H}, J=7.1,1.8 \mathrm{~Hz}), 7.34(\mathrm{td}, 1 \mathrm{H}, J=7.9,1.8 \mathrm{~Hz}$ ), $7.23-7.15$ (m, 4H), 7.08 (tt, $1 \mathrm{H}, J=5.7,3.1 \mathrm{~Hz}), 6.90$ (t, $1 \mathrm{H}, J=7.2 \mathrm{~Hz}), 6.79$ (d, $1 \mathrm{H}, J=8.3 \mathrm{~Hz})$, 4.34 (qd, 2H, $J=6.1,3.8 \mathrm{~Hz}), 3.76(\mathrm{~s}, 3 \mathrm{H}), 1.41$ (s, 3H), 1.26 (dd, 1H, $J=10.4,3.8 \mathrm{~Hz}), 0.77$ (dd, $1 \mathrm{H}, J=7.5,3.9 \mathrm{~Hz}), 0.39$ (ddt, $1 \mathrm{H}, J=11.1,7.5,3.8 \mathrm{~Hz}) .{ }^{13} \mathrm{C} \mathrm{NMR}\left(100 \mathrm{MHz}, \mathrm{CDCl}_{3}\right) \delta 164.2$, $148.3,137.1,131.8,128.2,126.8,125.6,121.3,120.8,109.5,55.3,25.2,23.2,20.5,8.4$. HRMS (TOF-MS ES ${ }^{+}$): $[\mathrm{M}+\mathrm{H}]^{+}$, calculated for $\mathrm{C}_{17} \mathrm{H}_{21} \mathrm{OSi}$ : 269.1356; found 269.1365.

\section{(2-phenyl-2-phenylcyclopropyl)(phenyl)silane (2n)}

Silycyclopropane $\mathbf{2 n}$ was prepared according to the general procedure 1. After standard work-up and purification by flash column (100\% Hexane), $2 \mathrm{n}$ was obtained as a colorless oil $(37.2 \mathrm{mg}, 62 \%$ yield). ${ }^{1} \mathrm{H}$ NMR (400 MHz, $\left.\mathrm{CDCl}_{3}\right) \delta 7.40-7.34(\mathrm{~m}, 4 \mathrm{H}), 7.30-7.11(\mathrm{~m}, 24 \mathrm{H}), 7.08-7.02(\mathrm{~m}$, 2H), $4.14(\mathrm{dd}, 2 \mathrm{H}, J=6.3,2.4 \mathrm{~Hz}), 3.76(\mathrm{dd}, 2 \mathrm{H}, J=6.3,4.4 \mathrm{~Hz}), 1.52(\mathrm{dd}, 2 \mathrm{H}, J=7.6,4.0 \mathrm{~Hz})$, $1.49-1.42(\mathrm{~m}, 3 \mathrm{H}), 1.02-0.92(\mathrm{~m}, 2 \mathrm{H}) .{ }^{13} \mathrm{C} \mathrm{NMR}\left(100 \mathrm{MHz}, \mathrm{CDCl}_{3}\right) \delta 146.9,142.5,135.3,132.3$, 130.4, 129.5, 128.2, 128.2, 127.9, 127.3, 126.7, 125.8, 35.3, 19.9, 9.4. HRMS (TOF-MS ES ${ }^{+}$): $[\mathrm{M}+\mathrm{H}]^{+}$, calculated for $\mathrm{C}_{21} \mathrm{H}_{21} \mathrm{Si}: 301.1407$; found 301.1399 .

\section{2-(4-Chlorophenyl)-2-methylcyclopropyl)diphenylsilane (2o)}

Silycyclopropane 2o was prepared according to the general procedure 1. After standard work-up and purification by flash column (100\% Hexane), 20 was obtained as a colorless oil $(62.6 \mathrm{mg}, 90 \%$ yield). ${ }^{1} \mathrm{H}$ NMR (400 MHz, $\left.\mathrm{CDCl}_{3}\right) \delta 7.70-7.62(\mathrm{~m}, 4 \mathrm{H}), 7.48-7.39(\mathrm{~m}, 6 \mathrm{H}), 7.29-7.22(\mathrm{~m}$, $4 \mathrm{H}), 4.95(\mathrm{~d}, 1 \mathrm{H}, J=5.6 \mathrm{~Hz}), 1.51-1.43(\mathrm{~m}, 4 \mathrm{H}), 1.06-0.99(\mathrm{~m}, 1 \mathrm{H}), 0.59$ (tdd, $1 \mathrm{H}, J=12.8$, 6.1, 3.4 Hz). ${ }^{13} \mathrm{C} \mathrm{NMR}\left(150 \mathrm{MHz}, \mathrm{CDCl}_{3}\right) \delta 146.6,135.8,135.3,135.1,135.1,134.8,134.4,131.4$, 129.6, 128.3, 128.2, 128.1, 128.0, 25.1, 23.6, 20.1, 11.2. HRMS (TOF-MS ES $\left.{ }^{+}\right):[\mathrm{M}+\mathrm{H}]^{+}$, calculated for $\mathrm{C}_{22} \mathrm{H}_{22} \mathrm{ClSi}$ : 349.1174; found 349.1182.

\section{2-(4-Methoxyphenyl)-2-methylcyclopropyl)diphenylsilane (2p)}

Silycyclopropane $\mathbf{2 p}$ was prepared according to the general procedure 1. After standard work-up and purification by flash column (100\% Hexane), $\mathbf{2 p}$ was obtained as a colorless oil $(60.5 \mathrm{mg}, 88 \%$ yield). ${ }^{1} \mathrm{H}$ NMR (400 MHz, $\left.\mathrm{CDCl}_{3}\right) \delta 7.69$ (ddd, 4H, $\left.J=19.1,7.8,1.6 \mathrm{~Hz}\right), 7.50-7.38(\mathrm{~m}, 6 \mathrm{H})$, $7.26(\mathrm{~d}, 2 \mathrm{H}, J=8.7 \mathrm{~Hz}), 6.86(\mathrm{~d}, 2 \mathrm{H}, J=8.7 \mathrm{~Hz}), 4.95(\mathrm{~d}, 1 \mathrm{H}, J=5.6 \mathrm{~Hz}), 3.82(\mathrm{~s}, 3 \mathrm{H}), 1.48$ (s, $3 \mathrm{H}), 1.43(\mathrm{dd}, 1 \mathrm{H}, J=10.3,3.8 \mathrm{~Hz}), 0.98$ (dd, $1 \mathrm{H}, J=7.5,3.8 \mathrm{~Hz}), 0.59$ (ddd, $1 \mathrm{H}, J=10.2,7.4$, $5.6 \mathrm{~Hz}) .{ }^{13} \mathrm{C}$ NMR $\left(150 \mathrm{MHz}, \mathrm{CDCl}_{3}\right) \delta 157.6,140.4,135.3,135.1,134.8,129.5,129.5,128.0$, 128.0, 113.6, 55.3, 25.1, 24.2, 19.8, 10.4. HRMS (TOF-MS ES ${ }^{+}$: $[\mathrm{M}+\mathrm{H}]^{+}$, calculated for $\mathrm{C}_{23} \mathrm{H}_{25} \mathrm{OSi}$ : 345.1669; found 345.1665.

\section{2-(4-Methylphenyl)-2-methylcyclopropyl)diphenylsilane (2q)}

Silycyclopropane $\mathbf{2 q}$ was prepared according to the general procedure 1. After standard work-up and purification by flash column (100\% Hexane), 2 q was obtained as a colorless oil $(51.2 \mathrm{mg}, 78 \%$ yield). ${ }^{1} \mathrm{H}$ NMR (400 MHz, $\left.\mathrm{CDCl}_{3}\right) \delta 7.63-7.52(\mathrm{~m}, 4 \mathrm{H}), 7.35-7.25(\mathrm{~m}, 6 \mathrm{H}), 7.11(\mathrm{~d}, 2 \mathrm{H}, J=$ $8.2 \mathrm{~Hz}), 7.00(\mathrm{~d}, 2 \mathrm{H}, J=8.0 \mathrm{~Hz}), 4.83(\mathrm{~d}, 1 \mathrm{H}, J=5.6 \mathrm{~Hz}), 2.22(\mathrm{~s}, 3 \mathrm{H}), 1.38(\mathrm{~s}, 3 \mathrm{H}), 1.34(\mathrm{dd}, 1 \mathrm{H}$, $J=10.3,3.8 \mathrm{~Hz}), 0.87(\mathrm{dd}, 1 \mathrm{H}, J=7.5,3.8 \mathrm{~Hz}), 0.50(\mathrm{ddd}, 1 \mathrm{H}, J=10.3,7.5,5.6 \mathrm{~Hz}) .{ }^{13} \mathrm{C}$ NMR $\left(100 \mathrm{MHz}, \mathrm{CDCl}_{3}\right) \delta 145.1,135.3,135.2,135.2,135.1,134.8,129.5,129.5,128.9,127.99,127.96$, 
126.7, 25.2, 23.9, 20.9, 20.0, 10.7. HRMS (TOF-MS ES ${ }^{+}$): $[\mathrm{M}+\mathrm{H}]^{+}$, calculated for $\mathrm{C}_{23} \mathrm{H}_{25} \mathrm{Si}$ : 329.1720 ; found 329.1725 .

\section{(2,2-Dimethylcyclopropyl)diphenylsilane (2r)}

Silycyclopropane $\mathbf{2 r}$ was prepared according to the general procedure 1. After standard work-up and purification by flash column (100\% Hexane), $2 \mathbf{r}$ was obtained as a colorless oil $(28.7 \mathrm{mg}, 57 \%$ yield). ${ }^{1} \mathrm{H}$ NMR (400 MHz, $\left.\mathrm{CDCl}_{3}\right) \delta 7.63-7.53(\mathrm{~m}, 4 \mathrm{H}), 7.40-7.29(\mathrm{~m}, 6 \mathrm{H}), 4.73(\mathrm{~d}, 1 \mathrm{H}, J=$ $5.9 \mathrm{~Hz}), 1.19$ (s, 3H), $1.10(\mathrm{~s}, 3 \mathrm{H}), 0.79$ (dd, $1 \mathrm{H}, J=10.0,3.5 \mathrm{~Hz}), 0.54(\mathrm{dd}, 1 \mathrm{H}, J=7.0,3.5 \mathrm{~Hz})$, $0.08-0(\mathrm{~m}, 1 \mathrm{H}) .{ }^{13} \mathrm{C} \mathrm{NMR}\left(100 \mathrm{MHz}, \mathrm{CDCl}_{3}\right) \delta 135.3,135.2,129.4,129.4,127.9,127.9,28.5$, 23.2, 19.6, 17.6, 8.7. HRMS (TOF-MS ES ${ }^{+}$): $[\mathrm{M}+\mathrm{H}]^{+}$, calculated for $\mathrm{C}_{17} \mathrm{H}_{21} \mathrm{Si}$ : 253.1407; found 253.1399 .

\subsection{General Procedure for the Co-catalyzed diastereo- and enantioselective}

\section{hydrosilylation of cyclopropenes (Procedure 2)}

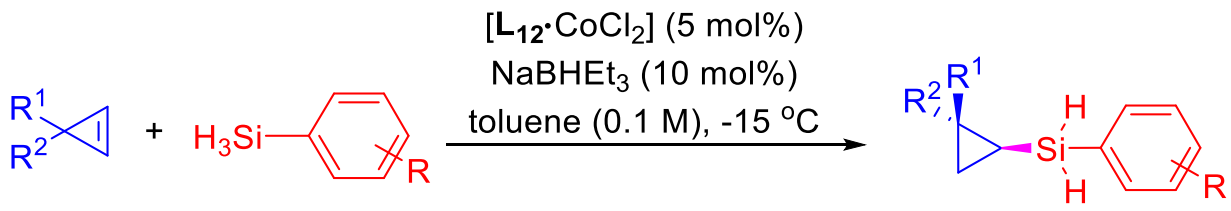

The $\left[\mathbf{L}_{12} \cdot \mathrm{CoCl}_{2}\right.$ ] complex (5.4 $\left.\mathrm{mg}, 0.010 \mathrm{mmol}, 5 \mathrm{~mol} \%\right)$ was added to a flask charged with a stirring bar, toluene $(2 \mathrm{~mL}, 0.1 \mathrm{M})$ under $\mathrm{N}_{2}$ atmosphere. After stirring at room temperature for $10 \mathrm{~min}$, the corresponding aryl silane $(0.40 \mathrm{mmol}, 2.0$ equiv.) was added to the flask, followed by the dropwise addition of the corresponding cyclopropene $(0.20$ mmol). After stirring at room temperature for $5 \mathrm{~min}$, the flask was cooled down to $-15^{\circ} \mathrm{C}$, and $\mathrm{NaBHEt}_{3}(1.0 \mathrm{M}$ in toluene, $20 \mu \mathrm{L}, 0.020 \mathrm{mmol}, 10 \mathrm{~mol} \%$ ) was added dropwise to the reaction mixture, which was further stirred for about $5 \mathrm{~h}$ until full conversion of cyclopropene (checked by analysis by TLC). The reaction mixture was evaporated directly and the oily residue was purified by flash column to afford the corresponding enantioenriched product.

\section{((1S,2R)-2-Methyl-2-phenylcyclopropyl)(phenyl)silane (2a)}

Silycyclopropane 2a was prepared according to the general procedure 2. After standard work-up and purification by flash column (100\% Hexane), 2a was obtained as a colorless oil (40.9 $\mathrm{mg}, 86 \%$ yield). Chiral HPLC (CHIRALCEL OD, Hexane: IPA = 2000:1, $1.0 \mathrm{~mL} / \mathrm{min}, 254 \mathrm{~nm}, \mathrm{t}_{\mathrm{R} 1}=6.7$ $\left.\min , \mathrm{t}_{\mathrm{R} 2}=7.0 \mathrm{~min}\right)$ indicated 96:04 er. $[\alpha]_{\mathrm{D}}{ }^{20}=-69.7\left(\mathrm{c}=0.10, \mathrm{CH}_{2} \mathrm{Cl}_{2}\right)$. 


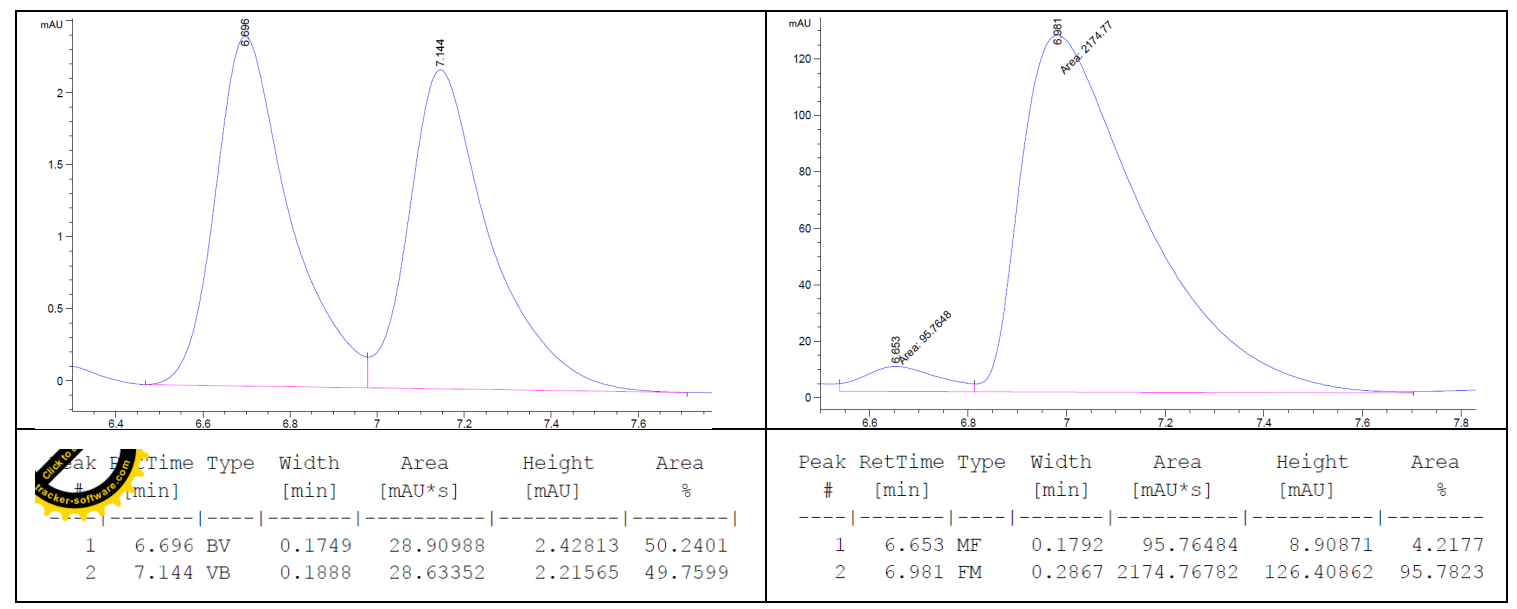

$2 \mathrm{mmol}$ scale reaction was performed under the same condition:

The $\left[\mathbf{L}_{12} \cdot \mathrm{CoCl}_{2}\right]$ complex (54 mg, $0.10 \mathrm{mmol}, 5 \mathrm{~mol} \%$ ) was added to a flask charged with a stirring bar, toluene $(20 \mathrm{~mL}, 0.1 \mathrm{M})$ under $\mathrm{N}_{2}$ atmosphere. After stirring at room temperature for $10 \mathrm{~min}$, the corresponding aryl silane ( $4.0 \mathrm{mmol}, 2.0$ equiv.) was added to the flask, followed by the dropwise addition of the corresponding cyclopropene $(260 \mathrm{mg}, 2.0 \mathrm{mmol})$. After stirring at room temperature for $5 \mathrm{~min}$, the flask was cooled down to $-15^{\circ} \mathrm{C}$, and $\mathrm{NaBHEt}_{3}(1.0 \mathrm{M}$ in toluene, 200 $\mu \mathrm{L}, 0.20 \mathrm{mmol}, 10 \mathrm{~mol} \%$ ) was added dropwise to the reaction mixture, which was further stirred for about $5 \mathrm{~h}$ until full conversion of cyclopropene (checked by analysis of TLC). The reaction mixture was evaporated directly and the oily residue was purified by flash column (100\% Hexane) to afford the silylated product $\mathbf{2 a}$ (357 $\mathrm{mg}, 75 \%$ yield, er 96:04).

\section{((1S,2R)-2-(4-Chlorophenyl)-2-methylcyclopropyl)(phenyl)silane (2b)}

Silycyclopropane $\mathbf{2 b}$ was prepared according to the general procedure 2. After standard work-up and purification by flash column (100\% Hexane), $2 \mathbf{b}$ was obtained as a colorless oil $(47.3 \mathrm{mg}, 87 \%$ yield). Chiral HPLC (CHIRALCEL OD, Hexane: IPA = 2000:1, $1.0 \mathrm{~mL} / \mathrm{min}, 254 \mathrm{~nm}, \mathrm{t}_{\mathrm{R} 1}=6.9$ $\left.\min , \mathrm{t}_{\mathrm{R} 2}=7.2 \mathrm{~min}\right)$ indicated 94:06 er. $[\alpha]_{\mathrm{D}}{ }^{20}=-64.1\left(\mathrm{c}=0.68, \mathrm{CH}_{2} \mathrm{Cl}_{2}\right)$.

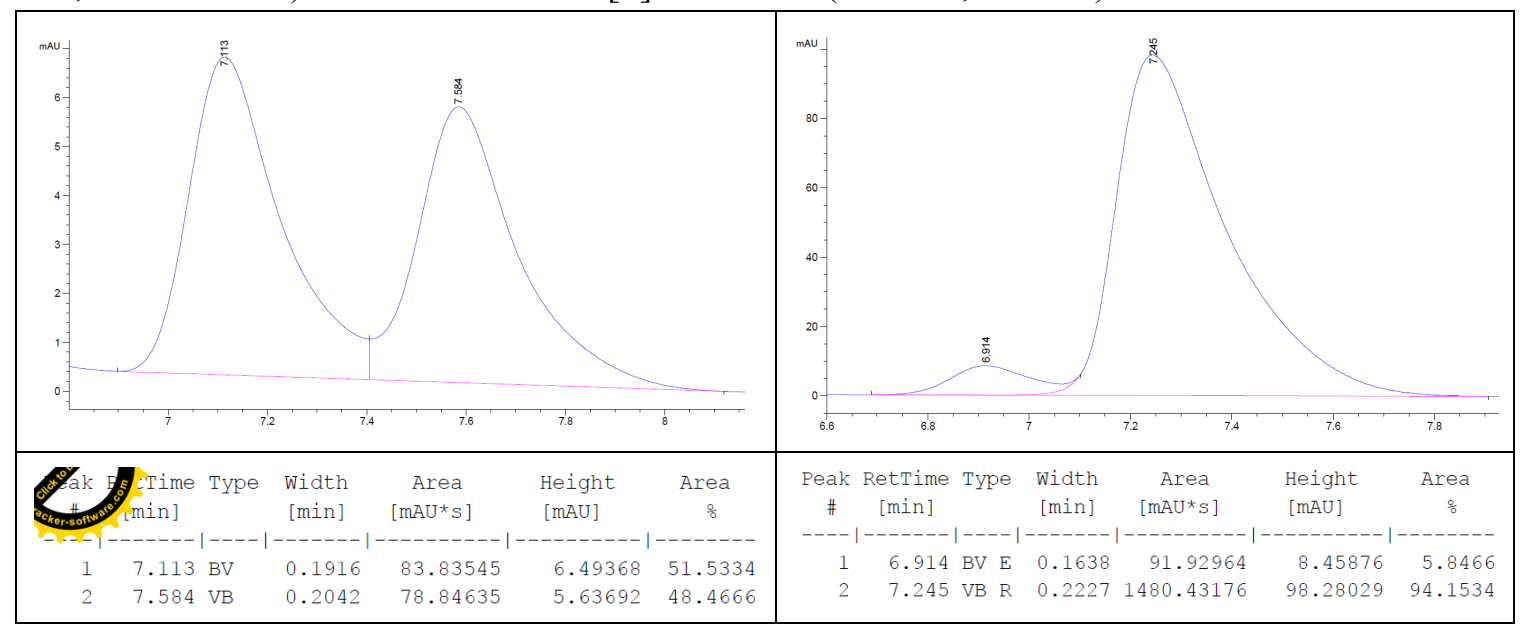

((1S,2R)-2-(4-Methoxyphenyl)-2-methylcyclopropyl)(phenyl)silane (2c) 
Silycyclopropane $\mathbf{2 c}$ was prepared according to the general procedure 2. After standard work-up and purification by flash column (100\% Hexane), $2 \mathbf{c}$ was obtained as a colorless oil $(44.5 \mathrm{mg}, 83 \%$ yield). Chiral HPLC (CHIRALCEL ODH, Hexane: IPA $=2000: 1,1.0 \mathrm{~mL} / \mathrm{min}, 254 \mathrm{~nm}, \mathrm{t}_{\mathrm{R} 1}=11.6$ $\left.\min , t_{\mathrm{R} 2}=13.1 \mathrm{~min}\right)$ indicated 95:05 er. $[\alpha]_{\mathrm{D}}{ }^{20}=-53.4\left(\mathrm{c}=0.81, \mathrm{CH}_{2} \mathrm{Cl}_{2}\right)$.

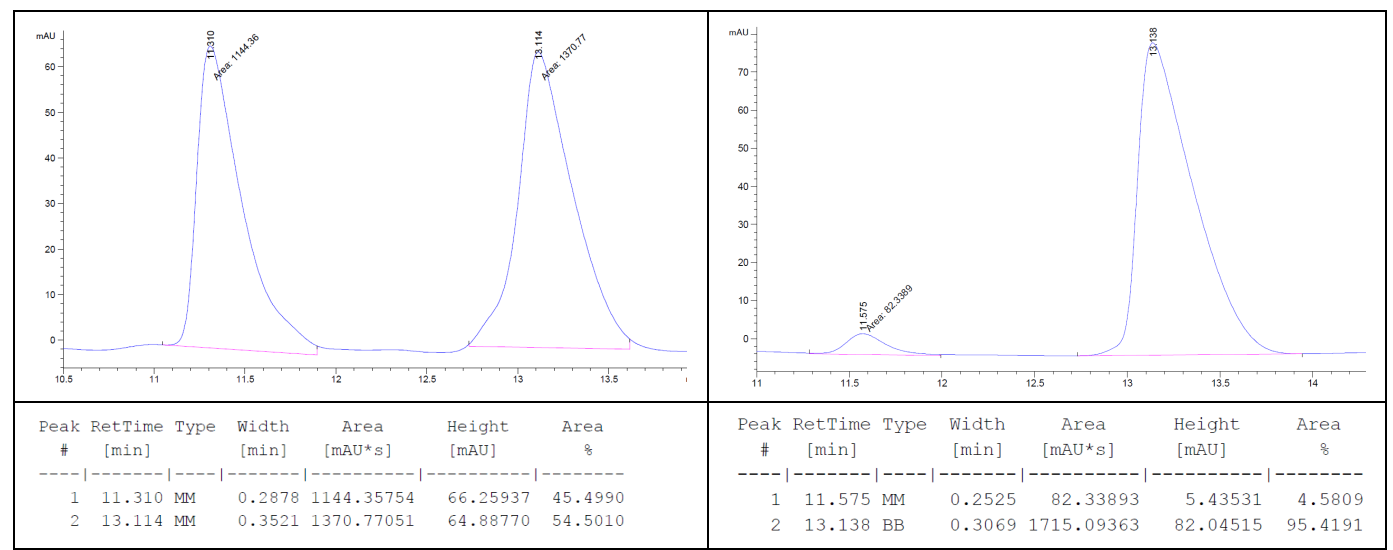

\section{((1S,2R)-2-Methyl-2-(p-tolyl)cyclopropyl)(phenyl)silane (2d)}

Silycyclopropane 2d was prepared according to the general procedure 2. After standard work-up and purification by flash column (100\% Hexane), 2d was obtained as a colorless oil (39.8 $\mathrm{mg}, 79 \%$ yield). Chiral HPLC (CHIRALCEL OBH, 100\% hexanes, $1.0 \mathrm{~mL} / \mathrm{min}, 254 \mathrm{~nm}, \mathrm{t}_{\mathrm{R} 1}=6.6 \mathrm{~min}, \mathrm{t}_{\mathrm{R} 2}$ $=7.5 \mathrm{~min})$ indicated 97:03 er. $[\alpha]_{\mathrm{D}}{ }^{20}=-61.7\left(\mathrm{c}=0.18, \mathrm{CH}_{2} \mathrm{Cl}_{2}\right)$.

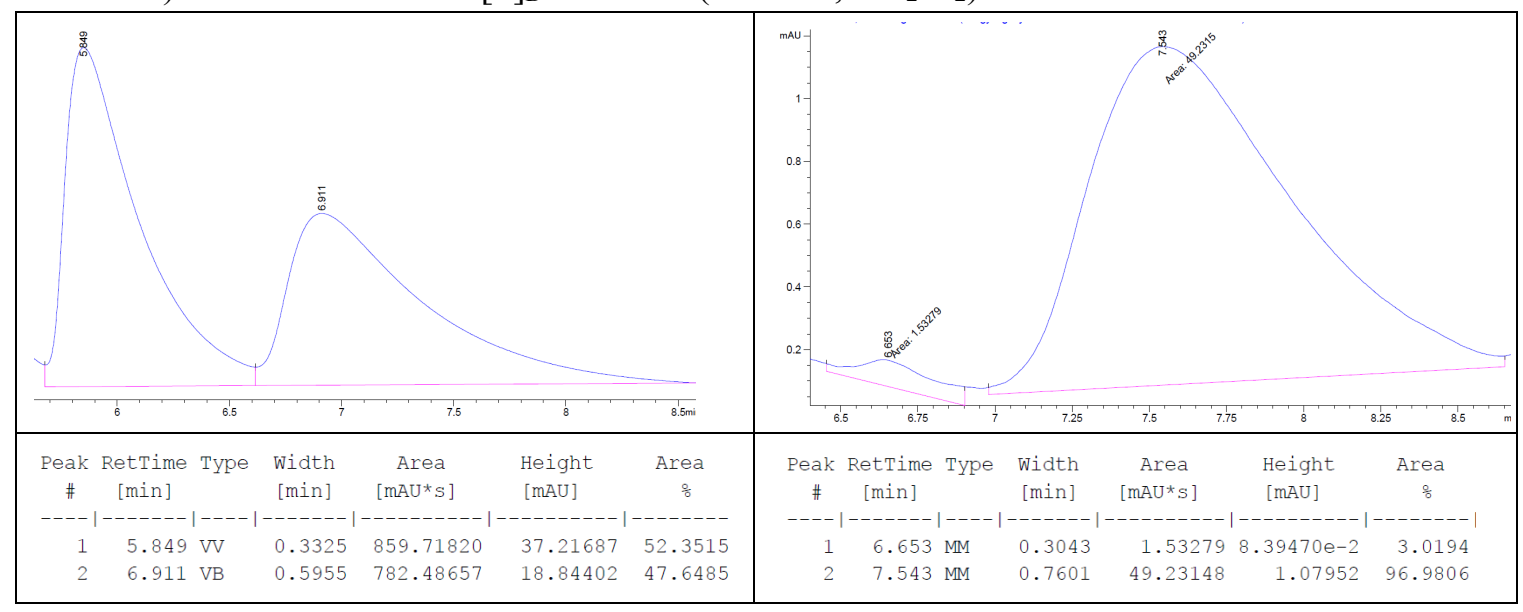

((1S,2R)-2-(4-Ethylphenyl)-2-methylcyclopropyl)(phenyl)silane (2e)

Silycyclopropane 2e was prepared according to the general procedure 2. After standard work-up and purification by flash column (100\% Hexane), $2 \mathbf{e}$ was obtained as a colorless oil $(38.1 \mathrm{mg}, 72 \%$ yield). ${ }^{1} \mathrm{H}$ NMR $\left(400 \mathrm{MHz}, \mathrm{CDCl}_{3}\right) \delta 7.72(\mathrm{dd}, 2 \mathrm{H}, J=7.3,1.8 \mathrm{~Hz}), 7.45(\mathrm{q}, 3 \mathrm{H}, J=6.0 \mathrm{~Hz}$ ), 7.26 $(\mathrm{d}, 2 \mathrm{H}, J=8.2 \mathrm{~Hz}), 7.17(\mathrm{~d}, 2 \mathrm{H}, J=8.2 \mathrm{~Hz}), 4.52(\mathrm{ddd}, 2 \mathrm{H}, J=18.2,6.0,3.9 \mathrm{~Hz}), 2.67(\mathrm{q}, 2 \mathrm{H}, J=$ $7.6 \mathrm{~Hz}), 1.56(\mathrm{~s}, 3 \mathrm{H}), 1.43(\mathrm{dd}, 1 \mathrm{H}, J=10.2,3.8 \mathrm{~Hz}), 1.28(\mathrm{t}, 3 \mathrm{H}, J=7.6 \mathrm{~Hz}), 0.92$ (dd, $1 \mathrm{H}, J=$ 7.4, $3.9 \mathrm{~Hz}), 0.45$ (ddd, $1 \mathrm{H}, J=14.3,7.5,3.8 \mathrm{~Hz}) .{ }^{13} \mathrm{C} \mathrm{NMR}\left(100 \mathrm{MHz}, \mathrm{CDCl}_{3}\right) \delta 145.1,141.7$, 135.3, 132.8, 129.6, 128.0, 127.7, 126.7, 28.4, 24.9, 23.6, 20.4, 15.6, 8.5. HRMS (TOF-MS ES ${ }^{+}$): $[\mathrm{M}+\mathrm{H}]^{+}$, calculated for $\mathrm{C}_{18} \mathrm{H}_{22} \mathrm{Si}$ : 266.1564; found 266.1566. Chiral HPLC (CHIRALCEL OD, 100\% 
hexanes, $\left.1.0 \mathrm{~mL} / \mathrm{min}, 254 \mathrm{~nm}, \mathrm{t}_{\mathrm{R} 1}=6.3 \mathrm{~min}, \mathrm{t}_{\mathrm{R} 2}=6.6 \mathrm{~min}\right)$ indicated $96: 04 \mathrm{er}$. $[\alpha]_{\mathrm{D}}{ }^{20}=-62.7(\mathrm{c}=$ $0.30, \mathrm{CH}_{2} \mathrm{Cl}_{2}$ ).

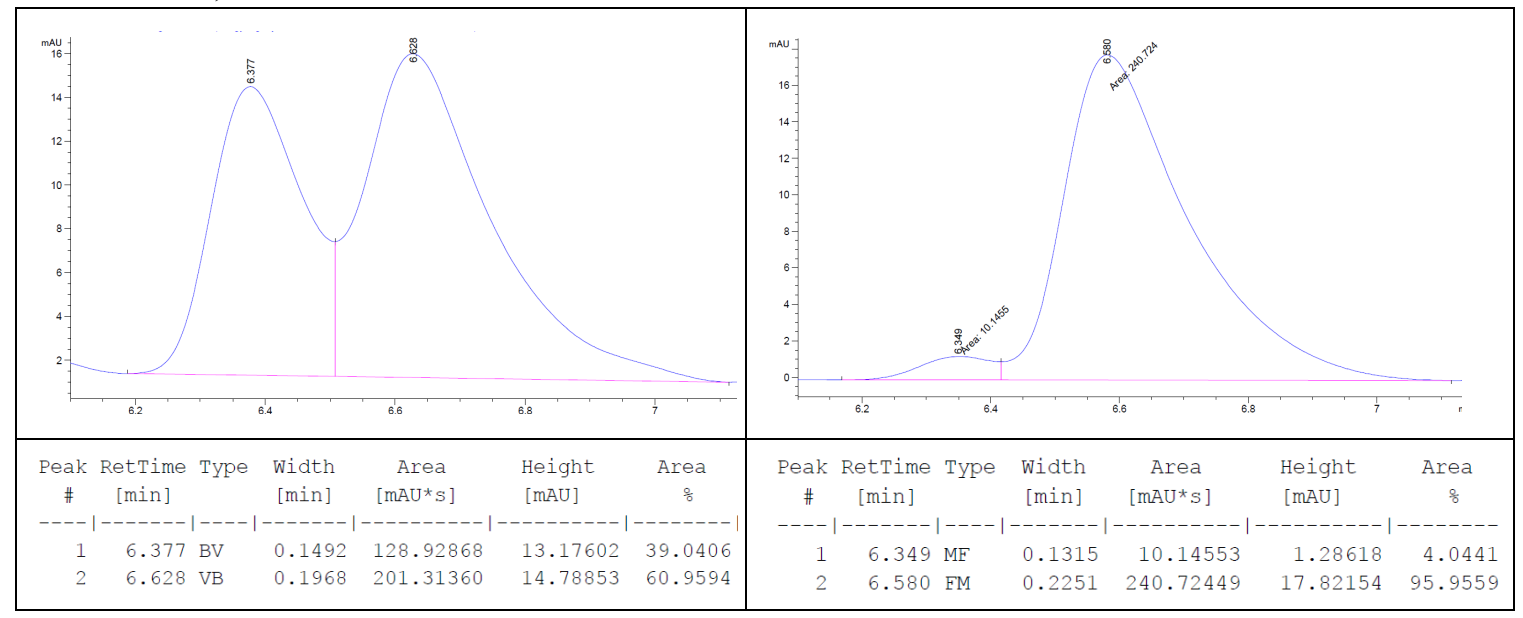

(4-Methoxyphenyl)((1S,2R)-2-methyl-2-phenylcyclopropyl)silane (2g)

Silycyclopropane $\mathbf{2 g}$ was prepared according to the general procedure 2. After standard work-up and purification by flash column (100\% Hexane), $2 \mathrm{~g}$ was obtained as a colorless oil (34.8 $\mathrm{mg}$, 65\% yield). ${ }^{1} \mathrm{H}$ NMR $\left(400 \mathrm{MHz}, \mathrm{CDCl}_{3}\right) \delta 7.50$ (d, $\left.2 \mathrm{H}, J=8.4 \mathrm{~Hz}\right), 7.20$ (t, $\left.4 \mathrm{H}, J=5.1 \mathrm{~Hz}\right), 7.09$ (dd, $1 \mathrm{H}, J=8.6,4.3 \mathrm{~Hz}), 6.86(\mathrm{~d}, 2 \mathrm{H}, J=8.4 \mathrm{~Hz}), 4.37$ (ddd, 2H, $J=17.8,6.0,3.8 \mathrm{~Hz}), 3.74(\mathrm{~s}, 3 \mathrm{H})$, 1.43 (s, 3H), $1.30(\mathrm{dd}, 1 \mathrm{H}, J=10.3,3.8 \mathrm{~Hz}), 0.79(\mathrm{dd}, 1 \mathrm{H}, J=7.4,3.9 \mathrm{~Hz}), 0.35-0.27(\mathrm{~m}, 1 \mathrm{H})$. ${ }^{13} \mathrm{C} \mathrm{NMR}\left(100 \mathrm{MHz}, \mathrm{CDCl}_{3}\right) \delta 160.9,148.0,136.7,128.2,126.8,125.7,123.2,113.9,55.0,25.2$, 23.5, 20.4, 8.9. HRMS (TOF-MS $\mathrm{ES}^{+}$): $[\mathrm{M}+\mathrm{H}]^{+}$, calculated for $\mathrm{C}_{17} \mathrm{H}_{21} \mathrm{OSi}$ : 269.1356; found 269.1369. Chiral HPLC (CHIRALCEL IA, Hexane: IPA $=2000: 1,1.0 \mathrm{~mL} / \mathrm{min}, 254 \mathrm{~nm}, \mathrm{t}_{\mathrm{R} 1}=15.6$ $\left.\min , \mathrm{t}_{\mathrm{R} 2}=16.8 \mathrm{~min}\right)$ indicated 99:01 er. $[\alpha]_{\mathrm{D}}{ }^{20}=-56.7\left(\mathrm{c}=0.20, \mathrm{CH}_{2} \mathrm{Cl}_{2}\right)$.

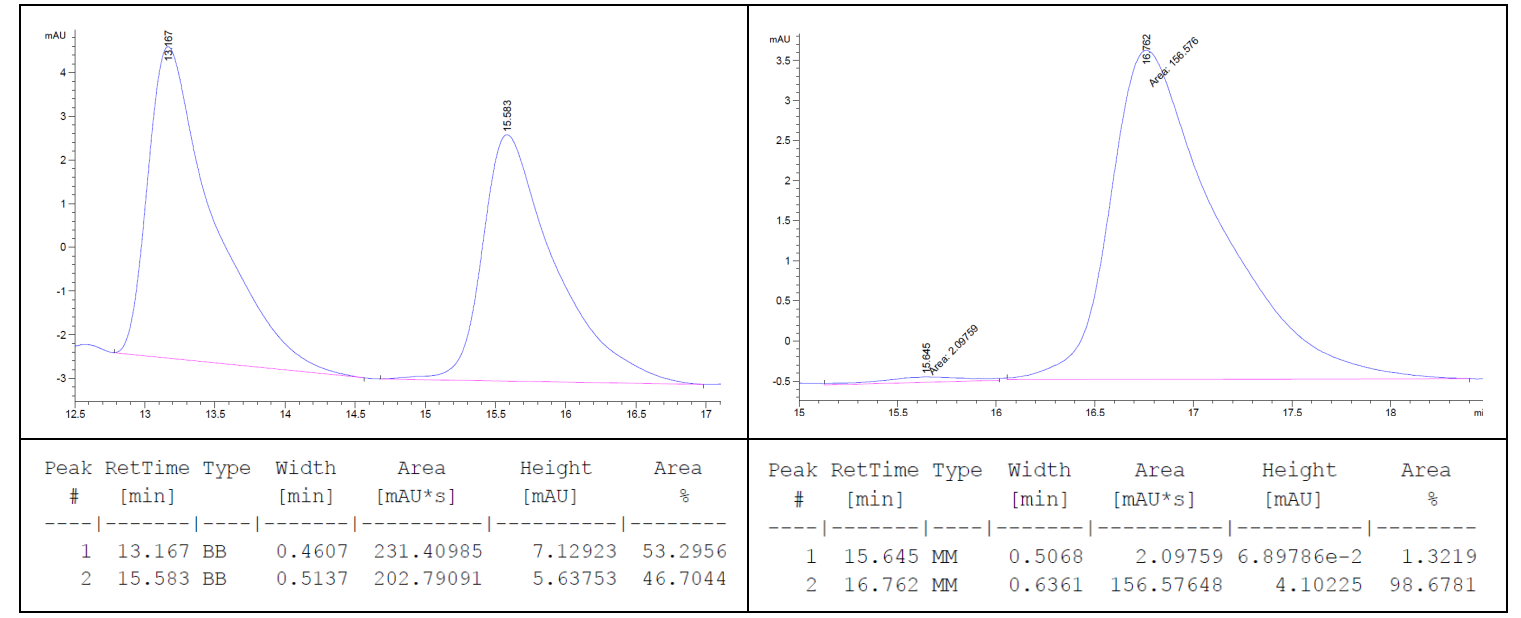

2-Methyl-2-phenylcyclopropyl)(p-tolyl)silane (2j)

Silycyclopropane $\mathbf{2} \mathbf{j}$ was prepared according to the general procedure 2. After standard work-up and purification by flash column (100\% Hexane), $\mathbf{2} \mathbf{j}$ was obtained as a colorless oil (33.8 $\mathrm{mg}, 67 \%$ yield). Chiral HPLC (CHIRALCEL IA, Hexane: IPA $=2000: 1,1.0 \mathrm{~mL} / \mathrm{min}, 254 \mathrm{~nm}, \mathrm{t}_{\mathrm{R} 1}=6.9 \mathrm{~min}$, $\left.\mathrm{t}_{\mathrm{R} 2}=7.3 \mathrm{~min}\right)$ indicated 93:07 er. $[\alpha]_{\mathrm{D}}^{20}=-59.2\left(\mathrm{c}=0.23, \mathrm{CH}_{2} \mathrm{Cl}_{2}\right)$. 


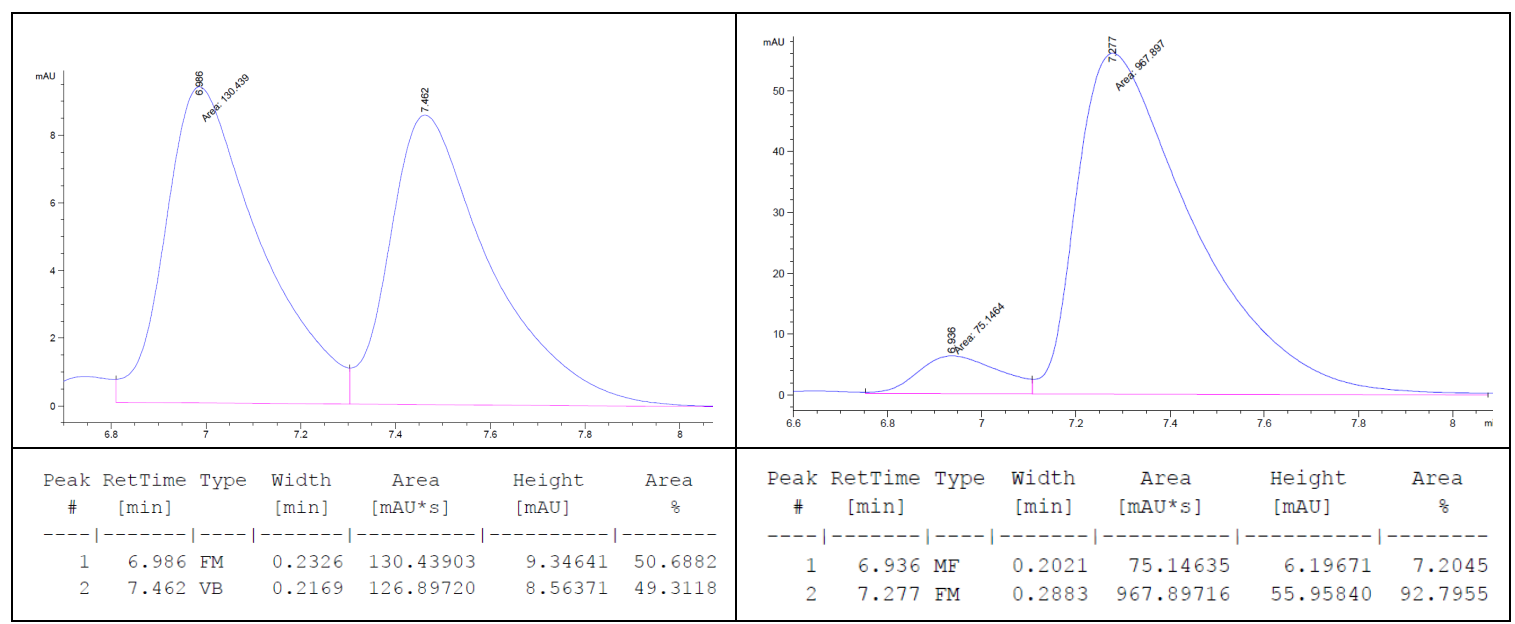

(2-Methoxyphenyl)((1S,2R)-2-methyl-2-phenylcyclopropyl)silane (2m)

Silycyclopropane $\mathbf{2} \mathbf{m}$ was prepared according to the general procedure 2. After standard work-up and purification by flash column (100\% Hexane), $\mathbf{2 m}$ was obtained as a colorless oil $(28.4 \mathrm{mg}, 53 \%$ yield). Chiral HPLC (CHIRALCEL OD, 100\% Hexane, $1.0 \mathrm{~mL} / \mathrm{min}, 254 \mathrm{~nm}, \mathrm{t}_{\mathrm{R} 1}=14.3 \mathrm{~min}, \mathrm{t}_{\mathrm{R} 2}=$ $15.8 \mathrm{~min})$ indicated $82: 18$ er. $[\alpha]_{\mathrm{D}}^{20}=-42.3\left(\mathrm{c}=0.43, \mathrm{CH}_{2} \mathrm{Cl}_{2}\right)$.

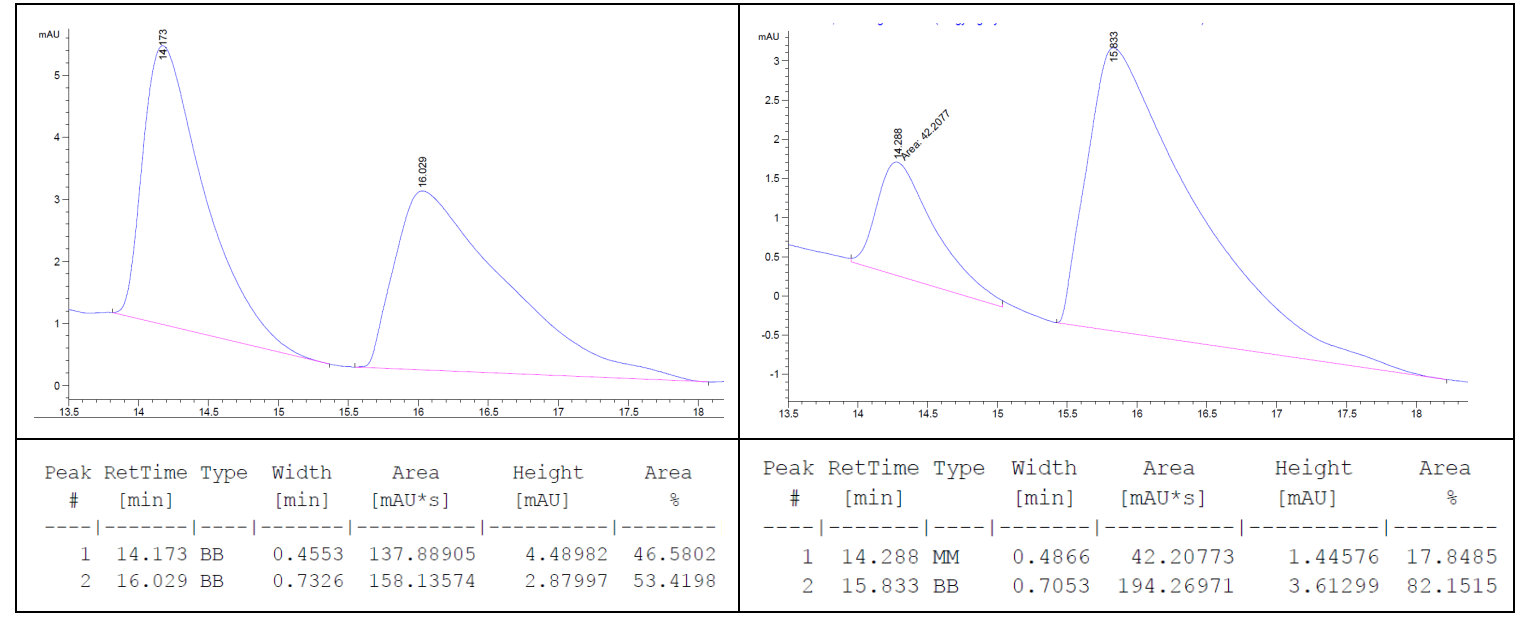

(4-Methoxyphenyl)((1S,2R)-2-methyl-2-(p-tolyl)cyclopropyl)silane (2s)

Silycyclopropane 2s was prepared according to the general procedure 2. After standard work-up and purification by flash column (100\% Hexane), $2 \mathrm{~s}$ was obtained as a colorless oil $(35.5 \mathrm{mg}, 63 \%$ yield). ${ }^{1} \mathrm{H}$ NMR (400 MHz, $\left.\mathrm{CDCl}_{3}\right) \delta 7.54-7.47(\mathrm{~m}, 2 \mathrm{H}), 7.13-7.07$ (m, 2H), 7.01 (d, 2H, $J=$ $7.9 \mathrm{~Hz}), 6.89-6.82(\mathrm{~m}, 2 \mathrm{H}), 4.38(\mathrm{dd}, 1 \mathrm{H}, J=6.0,3.9 \mathrm{~Hz}), 4.34(\mathrm{dd}, 1 \mathrm{H}, J=6.1,3.6 \mathrm{~Hz}), 3.75$ (s, $3 \mathrm{H}), 2.23(\mathrm{~s}, 3 \mathrm{H}), 1.41(\mathrm{~s}, 3 \mathrm{H}), 1.27(\mathrm{dd}, 1 \mathrm{H}, J=10.3,3.8 \mathrm{~Hz}), 0.77(\mathrm{dd}, 1 \mathrm{H}, J=7.4,3.8 \mathrm{~Hz}), 0.28$ (ddt, $1 \mathrm{H}, J=11.0,7.5,3.8 \mathrm{~Hz}) .{ }^{13} \mathrm{C}$ NMR $\left(100 \mathrm{MHz}, \mathrm{CDCl}_{3}\right) \delta 160.9,145.0,136.7,135.2,128.9$, 126.7, 123.3, 113.9, 55.0, 24.9, 23.6, 20.9, 20.3, 8.7. HRMS (TOF-MS ES ${ }^{+}$: $[\mathrm{M}+\mathrm{H}]^{+}$, calculated for $\mathrm{C}_{18} \mathrm{H}_{23} \mathrm{OSi}$ : 283.1513; found 283.1522. Chiral HPLC (CHIRALCEL OD, 100\% Hexane, 1.0 $\left.\mathrm{mL} / \mathrm{min}, 254 \mathrm{~nm}, \mathrm{t}_{\mathrm{R} 1}=14.7 \mathrm{~min}, \mathrm{t}_{\mathrm{R} 2}=18.4 \mathrm{~min}\right)$ indicated $92: 08 \mathrm{er} .[\alpha]_{\mathrm{D}}{ }^{20}=-51.3\left(\mathrm{c}=0.27, \mathrm{CH}_{2} \mathrm{Cl}_{2}\right)$. 


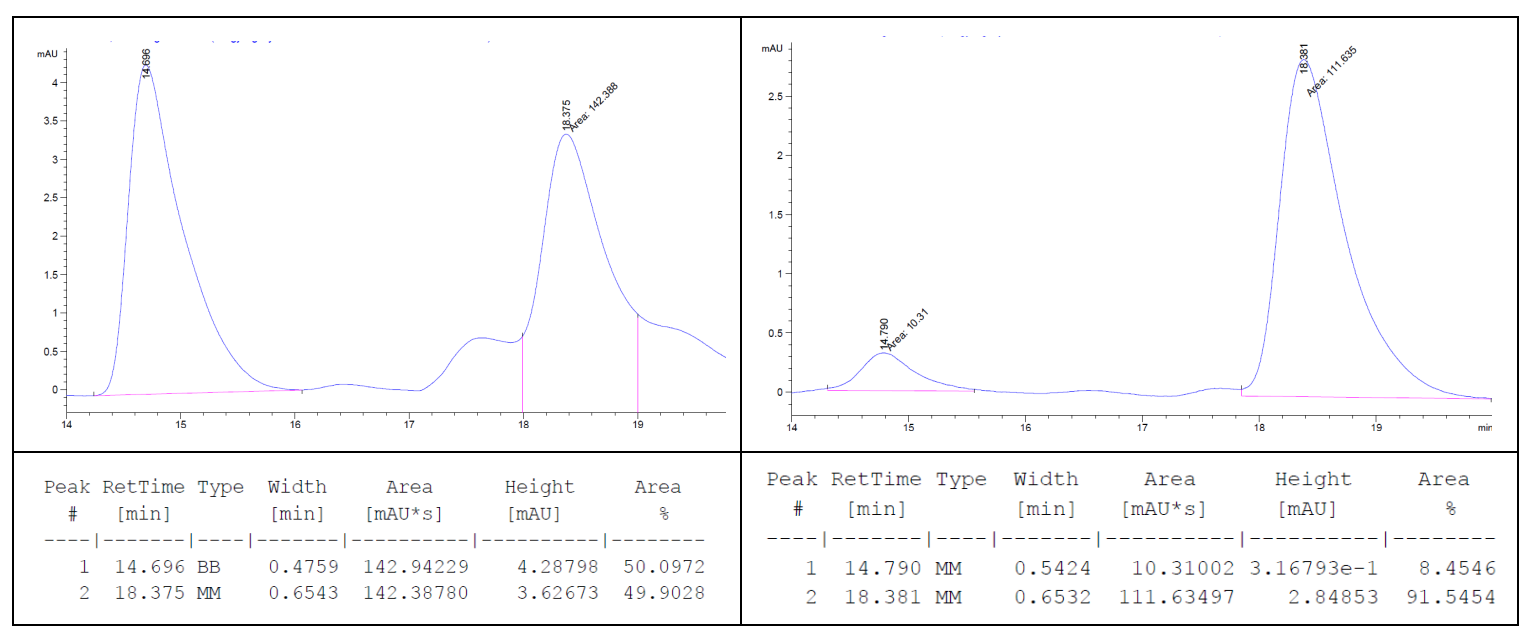

((1S,2R)-2-(4-Ethylphenyl)-2-methylcyclopropyl)(4-methoxyphenyl)silane (2t)

Silycyclopropane $\mathbf{2 t}$ was prepared according to the general procedure 2. After standard work-up and purification by flash column (100\% Hexane), $2 \mathbf{t}$ was obtained as a colorless oil (40.8 $\mathrm{mg}, 69 \%$ yield). ${ }^{1} \mathrm{H}$ NMR (400 MHz, $\left.\mathrm{CDCl}_{3}\right) \delta 7.50$ (d, $\left.2 \mathrm{H}, J=8.4 \mathrm{~Hz}\right), 7.12(\mathrm{~d}, 2 \mathrm{H}, J=8.2 \mathrm{~Hz}), 7.03$ (d, $2 \mathrm{H}, J=8.0 \mathrm{~Hz}), 6.86(\mathrm{~d}, 2 \mathrm{H}, J=8.5 \mathrm{~Hz}), 4.39(\mathrm{dd}, 1 \mathrm{H}, J=6.1,3.9 \mathrm{~Hz}), 4.34(\mathrm{dd}, 1 \mathrm{H}, J=6.1,3.6$ $\mathrm{Hz}), 3.75$ (s, 3H), 2.53 (q, 2H, $J=7.5 \mathrm{~Hz}), 1.41(\mathrm{~s}, 3 \mathrm{H}), 1.28$ (dd, $1 \mathrm{H}, J=10.3,3.9 \mathrm{~Hz}), 1.14$ (t, $4 \mathrm{H}, J=7.6 \mathrm{~Hz}), 0.77$ (dd, $1 \mathrm{H}, J=7.4,3.9 \mathrm{~Hz}), 0.29$ (td, $1 \mathrm{H}, J=7.2,3.7 \mathrm{~Hz}) .{ }^{13} \mathrm{C}$ NMR $(100 \mathrm{MHz}$, $\left.\mathrm{CDCl}_{3}\right) \delta 160.9,145.2,141.6,136.7,127.7,126.7,123.33,113.9,55.0,28.3,24.9,23.5,20.4,15.6$, 8.8. HRMS (TOF-MS ES ${ }^{+}$): $[\mathrm{M}+\mathrm{H}]^{+}$, calculated for $\mathrm{C}_{19} \mathrm{H}_{25} \mathrm{OSi}$ : 297.1669; found 297.1685. Chiral HPLC (CHIRALCEL OD, 100\% Hexane, $1.0 \mathrm{~mL} / \mathrm{min}, 254 \mathrm{~nm}, \mathrm{t}_{\mathrm{R} 1}=14.7 \mathrm{~min}, \mathrm{t}_{\mathrm{R} 2}=18.2 \mathrm{~min}$ ) indicated 91:09 er. $[\alpha]_{\mathrm{D}}^{20}=-50.2\left(\mathrm{c}=0.33, \mathrm{CH}_{2} \mathrm{Cl}_{2}\right)$.

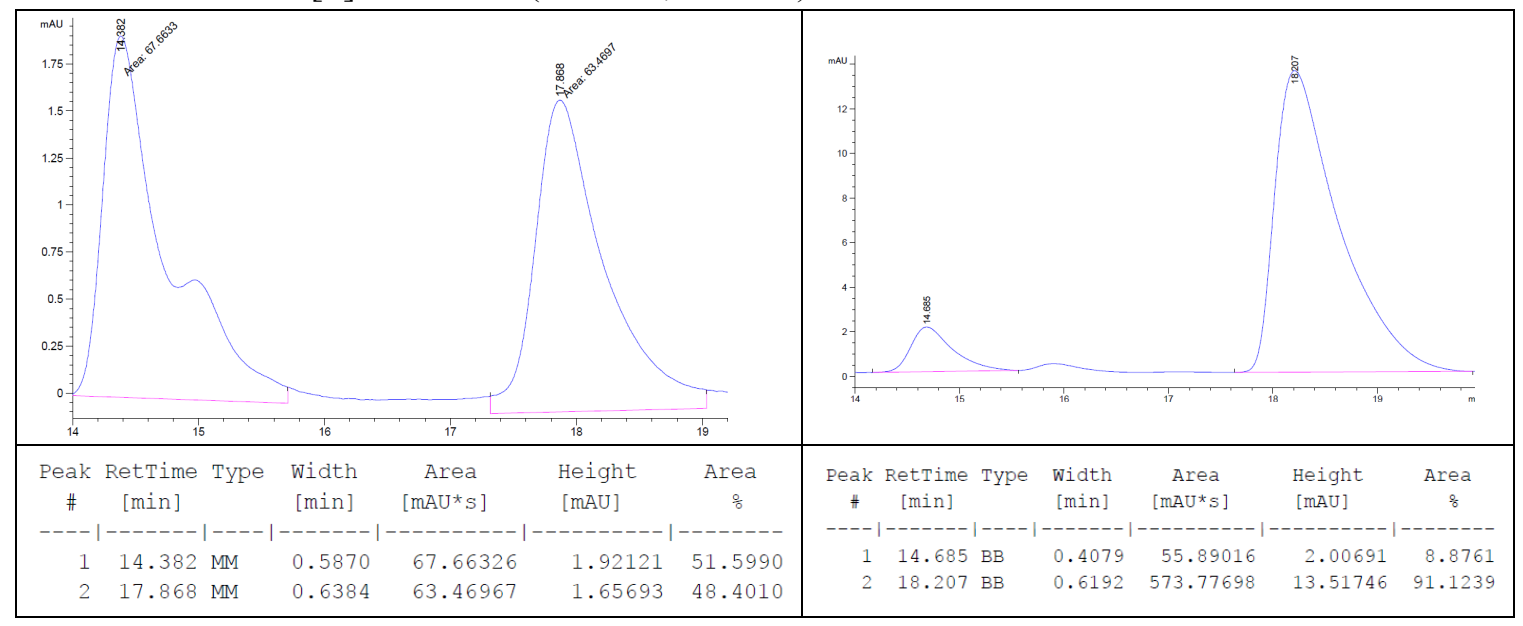

((1R,2S)-3',4'-Dihydro-2'H-spiro[cyclopropane-1,1'-naphthalen]-2-yl)(4methoxyphenyl)silane (2u)

Silycyclopropane $\mathbf{2 u}$ was prepared according to the general procedure 2. After standard work-up and purification by flash column (100\% Hexane), $2 \mathbf{u}$ was obtained as a colorless oil $(42.3 \mathrm{mg}, 72 \%$ yield). ${ }^{1} \mathrm{H}$ NMR (400 MHz, $\left.\mathrm{CDCl}_{3}\right) \delta 7.47$ (d, 2H, $\left.J=8.5 \mathrm{~Hz}\right), 7.04-6.94(\mathrm{~m}, 3 \mathrm{H}), 6.84(\mathrm{~d}, 2 \mathrm{H}, J$ $=8.5 \mathrm{~Hz}), 6.68(\mathrm{~d}, 1 \mathrm{H}, J=7.5 \mathrm{~Hz}), 4.40(\mathrm{dd}, 1 \mathrm{H}, J=6.2,3.0 \mathrm{~Hz}), 4.30(\mathrm{dd}, 1 \mathrm{H}, J=6.2,3.9 \mathrm{~Hz})$, 3.74 (s, 3H), 2.79 (t, $2 \mathrm{H}, J=6.1 \mathrm{~Hz}), 1.78$ (ddt, 4H, $J=12.2,9.6,5.2 \mathrm{~Hz}), 1.37$ (dd, $1 \mathrm{H}, J=10.3$, 
$4.1 \mathrm{~Hz}), 0.88(\mathrm{dd}, 1 \mathrm{H}, J=7.8,4.1 \mathrm{~Hz}), 0.41(\mathrm{ddt}, 1 \mathrm{H}, J=10.9,7.5,3.5 \mathrm{~Hz}) .{ }^{13} \mathrm{C} \mathrm{NMR}(100 \mathrm{MHz}$, $\left.\mathrm{CDCl}_{3}\right) \delta 160.9,142.0,137.5,136.8,128.9,126.1,124.9,123.0,121.5,113.9,55.0,32.6,30.6$, 24.1, 23.7, 22.5, 13.6. HRMS (TOF-MS ES ${ }^{+}$): $[\mathrm{M}+\mathrm{H}]^{+}$, calculated for $\mathrm{C}_{19} \mathrm{H}_{23} \mathrm{OSi}$ : 295.1513; found 297.1522. Chiral HPLC (CHIRALCEL OD, 100\% Hexane, $1.0 \mathrm{~mL} / \mathrm{min}, 254 \mathrm{~nm}, \mathrm{t}_{\mathrm{R} 1}=25.4 \mathrm{~min}$, $\left.\mathrm{t}_{\mathrm{R} 2}=32.9 \mathrm{~min}\right)$ indicated 96:04 er. $[\alpha]_{\mathrm{D}}{ }^{20}=-61.5\left(\mathrm{c}=0.40, \mathrm{CH}_{2} \mathrm{Cl}_{2}\right)$.

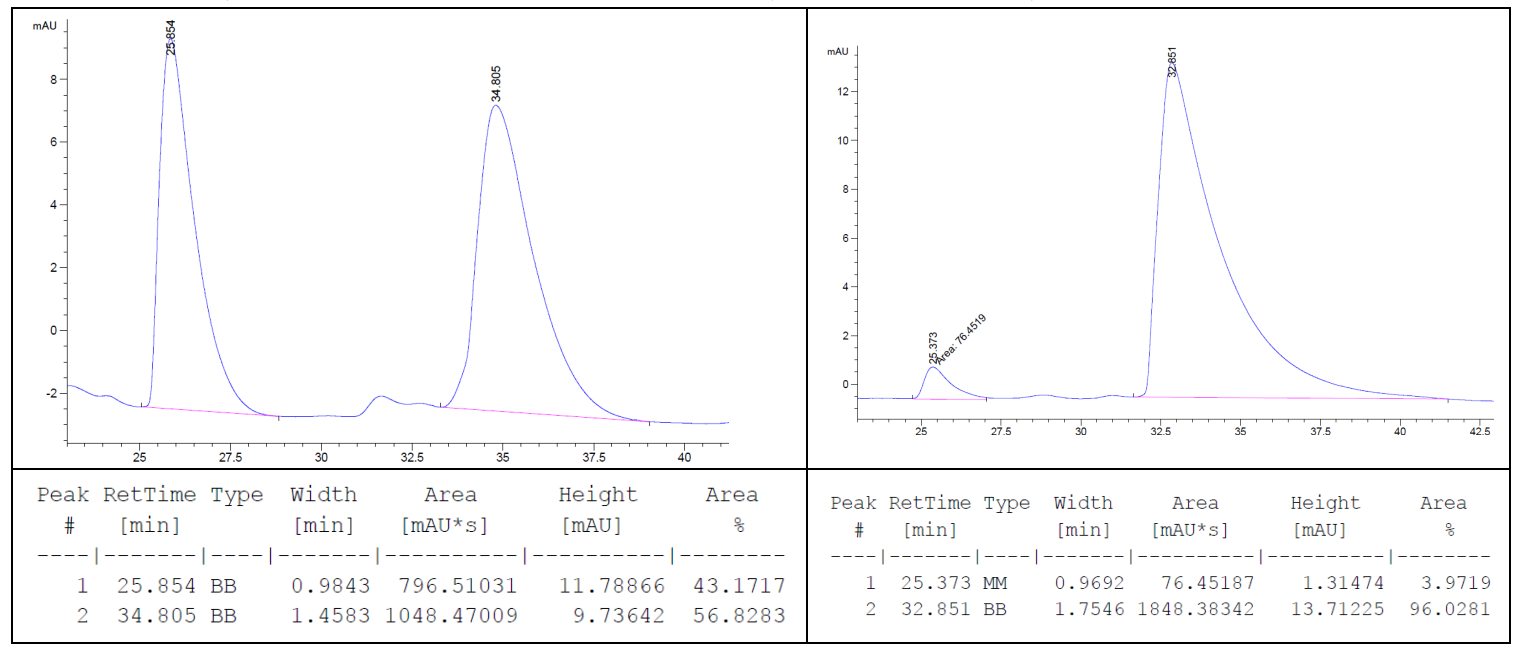

((1S,2R)-2-(4-Methoxyphenyl)-2-methylcyclopropyl)(p-tolyl)silane (2v)

Silycyclopropane $\mathbf{2} \mathbf{v}$ was prepared according to the general procedure 2. After standard work-up and purification by flash column (100\% Hexane), $2 \mathbf{v}$ was obtained as a colorless oil (44.0 $\mathrm{mg}, 78 \%$ yield). ${ }^{1} \mathrm{H}$ NMR (400 MHz, $\left.\mathrm{CDCl}_{3}\right) \delta 7.48(\mathrm{~d}, 2 \mathrm{H}, J=7.8 \mathrm{~Hz}), 7.12(\mathrm{dd}, 5 \mathrm{H}, J=8.3,3.0 \mathrm{~Hz}), 6.73$ $(\mathrm{d}, 2 \mathrm{H}, J=8.7 \mathrm{~Hz}), 4.38(\mathrm{dd}, 1 \mathrm{H}, J=6.0,3.9 \mathrm{~Hz}), 4.34(\mathrm{dd}, 1 \mathrm{H}, J=6.0,3.7 \mathrm{~Hz}), 3.69$ (s, 3H), 2.29 (s, 3H), 1.39 (s, 3H), 1.24 (dd, 1H, $J=10.3,3.9 \mathrm{~Hz}), 0.75$ (dd, 1H, $J=7.4,3.8 \mathrm{~Hz}), 0.26$ (ddt, 1H, $J=11.0,7.5,3.8 \mathrm{~Hz}) .{ }^{13} \mathrm{C} \mathrm{NMR}\left(100 \mathrm{MHz}, \mathrm{CDCl}_{3}\right) \delta 157.6,140.3,139.5,135.8,135.3,128.9$, 128.0, 113.6, 55.2, 24.8, 23.9, 21.5, 20.1, 8.2. HRMS (TOF-MS ES ${ }^{+}$): $[\mathrm{M}+\mathrm{H}]^{+}$, calculated for $\mathrm{C}_{18} \mathrm{H}_{23} \mathrm{OSi}$ : 283.1513; found 283.1541.Chiral HPLC (CHIRALCEL OD, 100\% Hexane, 1.0 $\left.\mathrm{mL} / \mathrm{min}, 254 \mathrm{~nm}, \mathrm{t}_{\mathrm{R} 1}=16.6 \mathrm{~min}, \mathrm{t}_{\mathrm{R} 2}=20.9 \mathrm{~min}\right)$ indicated $93: 07 \mathrm{er} .[\alpha]_{\mathrm{D}}{ }^{20}=-61.5\left(\mathrm{c}=0.25, \mathrm{CH}_{2} \mathrm{Cl}_{2}\right)$.

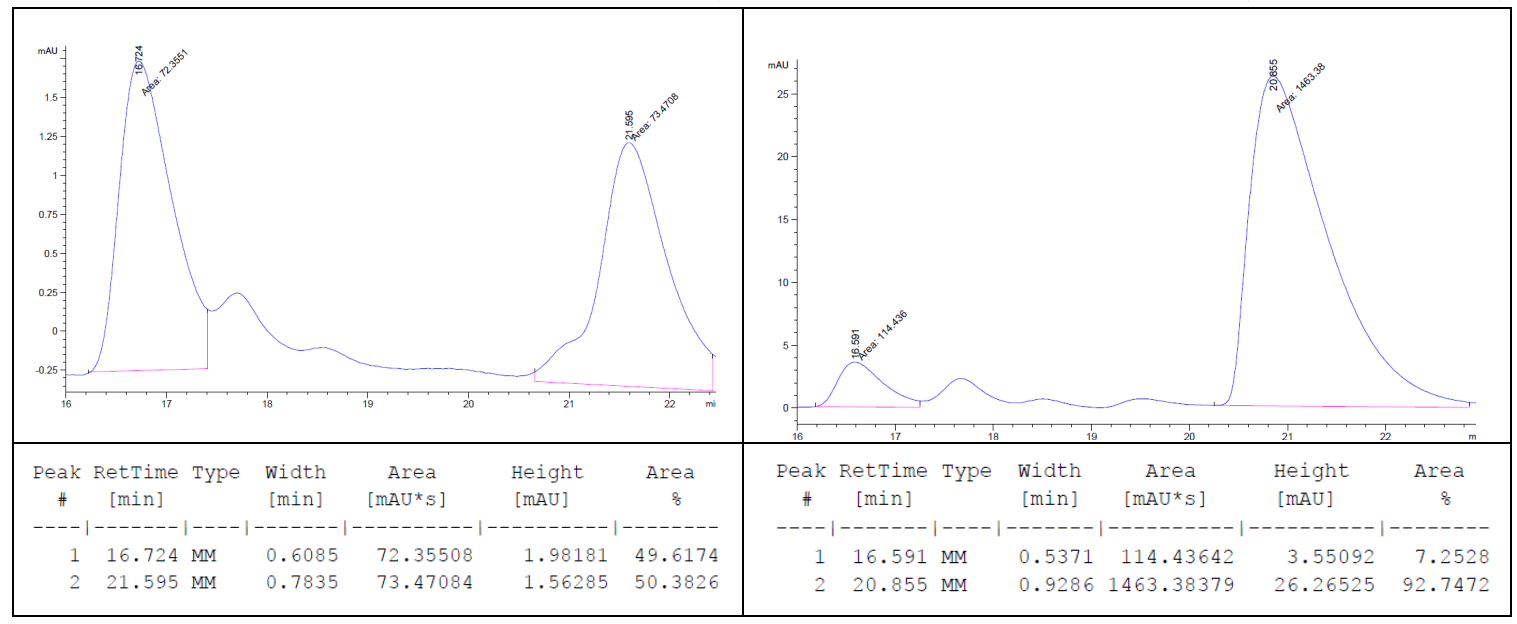

((1R,2S)-3',4'-Dihydro-2'H-spiro[cyclopropane-1,1'-naphthalen]-2-yl)(p-tolyl)silane (2w)

Silycyclopropane $\mathbf{2} \mathbf{w}$ was prepared according to the general procedure 2. After standard work-up and purification by flash column (100\% Hexane), 2 w was obtained as a colorless oil $(28.4 \mathrm{mg}, 51 \%$ 
yield). ${ }^{1} \mathrm{H}$ NMR ( $\left.400 \mathrm{MHz}, \mathrm{CDCl}_{3}\right) \delta 7.44(\mathrm{~d}, 2 \mathrm{H}, J=7.5 \mathrm{~Hz}), 7.11(\mathrm{~d}, 2 \mathrm{H}, J=7.5 \mathrm{~Hz}), 7.04-6.94$ $(\mathrm{m}, 3 \mathrm{H}), 6.68(\mathrm{~d}, 1 \mathrm{H}, J=7.5 \mathrm{~Hz}), 4.41(\mathrm{dd}, 1 \mathrm{H}, J=6.3,3.0 \mathrm{~Hz}), 4.30(\mathrm{dd}, 1 \mathrm{H}, J=6.2,4.1 \mathrm{~Hz})$, $2.79(\mathrm{t}, 2 \mathrm{H}, J=6.1 \mathrm{~Hz}), 2.28(\mathrm{~s}, 3 \mathrm{H}), 1.86-1.73(\mathrm{~m}, 4 \mathrm{H}), 1.38(\mathrm{dd}, 1 \mathrm{H}, J=10.3,4.1 \mathrm{~Hz}), 0.88(\mathrm{dd}$, $1 \mathrm{H}, J=7.8,4.1 \mathrm{~Hz}), 0.42(\mathrm{ddt}, 1 \mathrm{H}, J=11.0,7.5,3.5 \mathrm{~Hz}) \cdot{ }^{13} \mathrm{C} \mathrm{NMR}\left(100 \mathrm{MHz}, \mathrm{CDCl}_{3}\right) \delta 141.9$, 139.6, 137.5, 135.4, 128.9, 128.9, 128.7, 126.1, 124.9, 121.6, 32.6, 30.6, 24.1, 23.7, 22.5, 21.5, 13.5. HRMS (TOF-MS ES ${ }^{+}$): $[\mathrm{M}+\mathrm{H}]^{+}$, calculated for $\mathrm{C}_{19} \mathrm{H}_{23} \mathrm{Si}$ : 279.1564; found 279.1562. Chiral HPLC (CHIRALCEL OD, 100\% Hexane, $1.0 \mathrm{~mL} / \mathrm{min}, 254 \mathrm{~nm}, \mathrm{t}_{\mathrm{R} 1}=9.7 \mathrm{~min}, \mathrm{t}_{\mathrm{R} 2}=11.2 \mathrm{~min}$ ) indicated 98:02 er. $[\alpha]_{\mathrm{D}}^{20}=-54.5\left(\mathrm{c}=0.33, \mathrm{CH}_{2} \mathrm{Cl}_{2}\right)$.

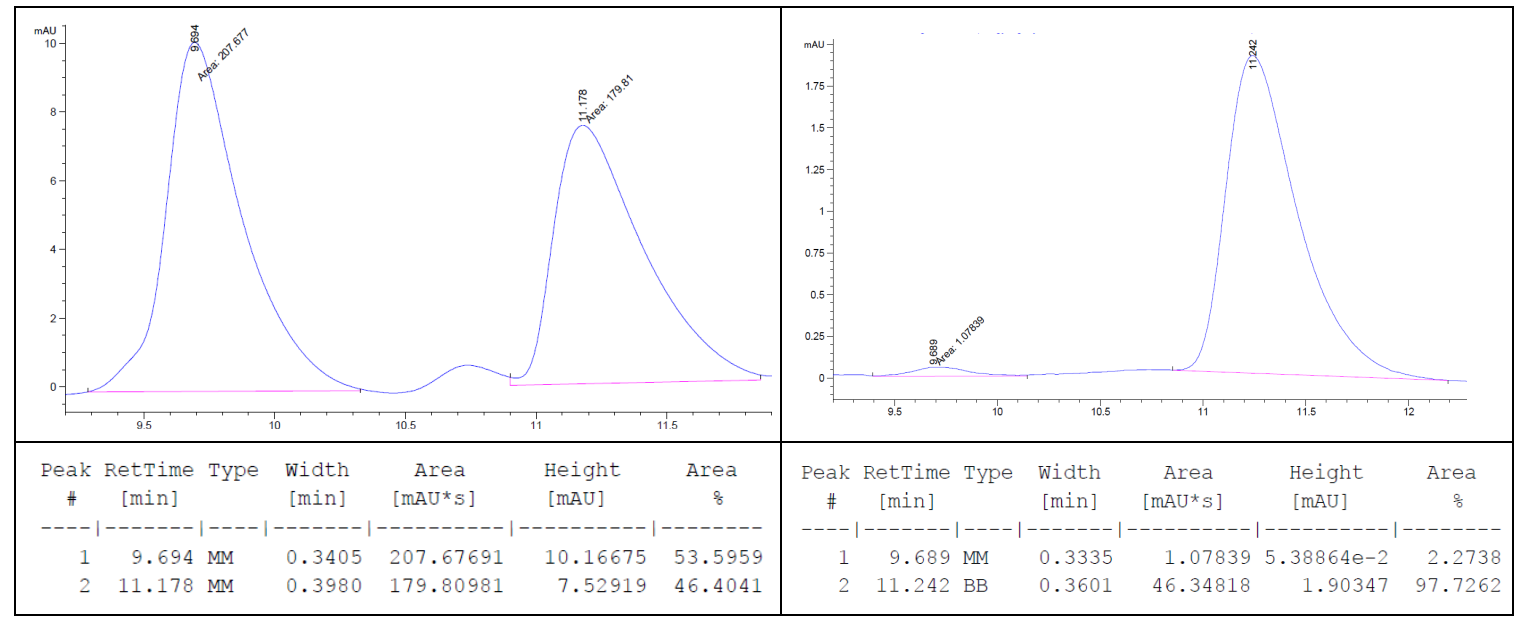

\subsection{Labelling experiments}

$\mathbf{2} \mathbf{g}_{\mathbf{d} 2}$ Silycyclopropane $\mathbf{2} \mathbf{g}_{\mathrm{d} 2}$ was prepared according to the general procedure 2 with the using cyclopropene $1 \mathbf{a}_{\mathrm{d} 2}$ and $p \mathrm{OMeC}_{6} \mathrm{H}_{4} \mathrm{SiH}_{3}$ as silane reagent. After standard work-up and purification by flash column (100\% Hexane), $\mathbf{2 g}_{\mathrm{d} 2}$ was obtained as a colorless oil ( $6.5 \mathrm{mg}, 12 \%$ yield). HRMS (TOF-MS ES ${ }^{+}$): $[\mathrm{M}+\mathrm{H}]^{+}$, calculated for $\mathrm{C}_{17} \mathrm{H}_{19} \mathrm{D}_{2} \mathrm{OSi}$ : 271.1482; found 271.1491 .

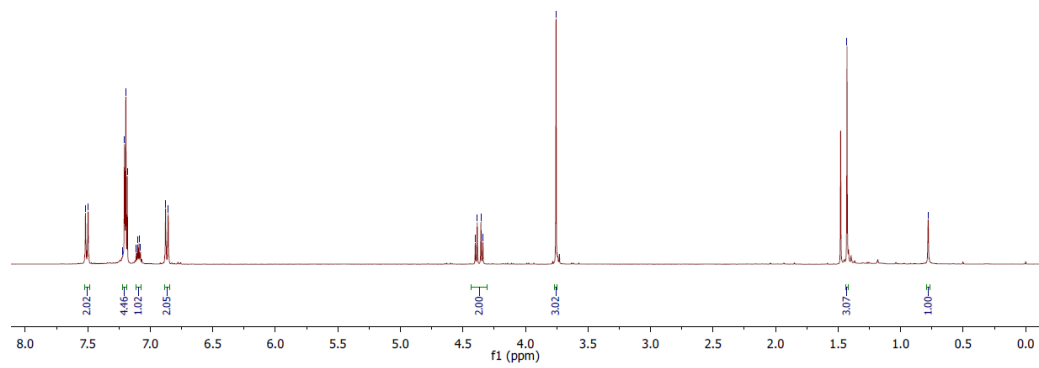


Silycyclopropane $\mathbf{2} \mathbf{g}_{\mathbf{d} 5}$ was prepared according to the general procedure 2 with the using cyclopropene $1 \mathbf{a}_{\mathbf{d} 2}$ and $p \mathrm{OMeC}_{6} \mathrm{H}_{4} \mathrm{SiD}_{3}$ as silane reagent. After standard work-up and purification by flash column (100\% Hexane), $\mathbf{2}_{\mathrm{d} 2}$ was obtained as a colorless oil (5.5 $\mathrm{mg}, 10 \%$ yield). HRMS (TOF-MS ES ${ }^{+}$): $[\mathrm{M}+\mathrm{H}]^{+}$, calculated for $\mathrm{C}_{17} \mathrm{H}_{16} \mathrm{D}_{5} \mathrm{OSi}$ : 274.1670; found 274.1675.
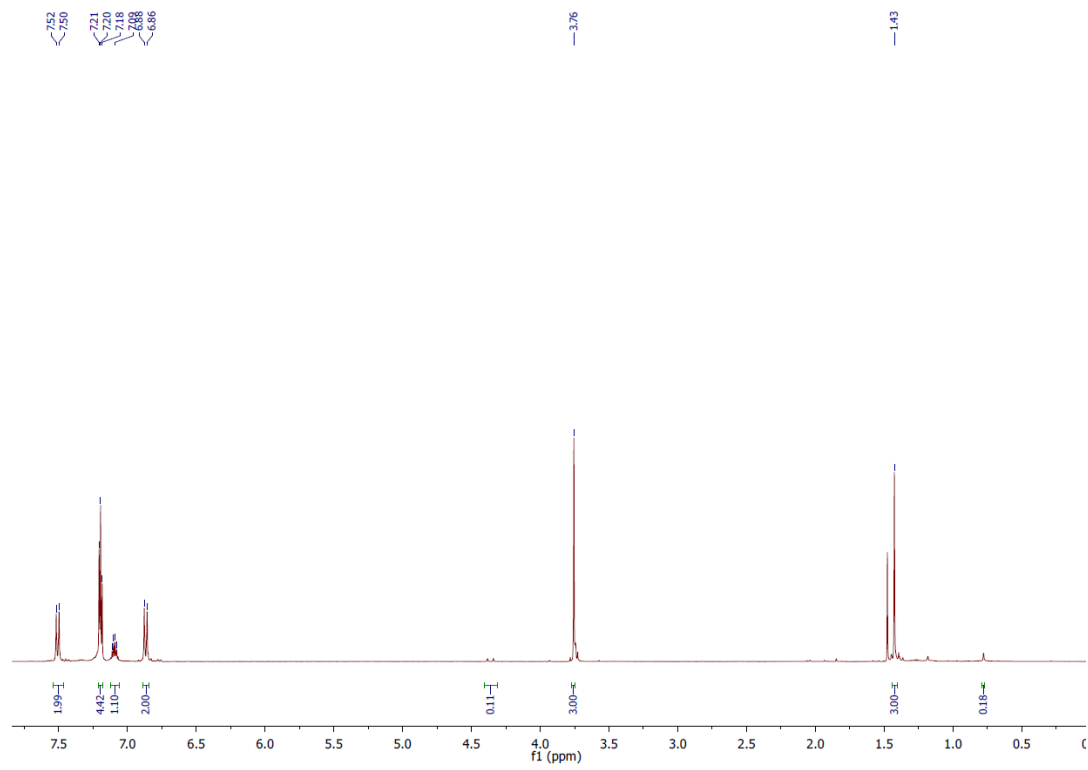

\subsection{Confirmation of the absolute configuration}

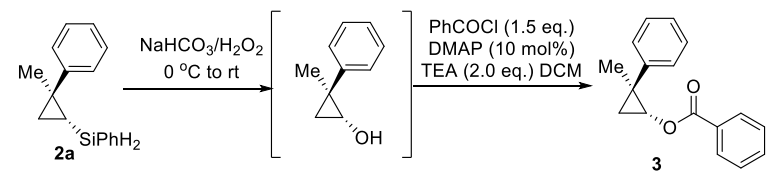

The oxidation step followed a reported procedure: ${ }^{5} \mathrm{NaHCO}_{3}(210 \mathrm{mg}, 2.5 \mathrm{mmol})$ and $30 \%$ aqueous $\mathrm{H}_{2} \mathrm{O}_{2}(1.0 \mathrm{~mL})$ were added into a mixture of THF $(1.0 \mathrm{~mL})$ and methanol $(1.0 \mathrm{~mL})$ in a $5 \mathrm{~mL}$ screw-capped. After stirring for $5 \mathrm{~min}$, 2a (60 mg, $0.25 \mathrm{mmol}$, er 96:04) was added dropwise slowly into the reaction mixture at $0{ }^{\circ} \mathrm{C}$ and then stirred at room temperature for 6 hours. Sodium thiosulfate was then added to quench the excess of $\mathrm{H}_{2} \mathrm{O}_{2}$. The mixture was extracted with $\mathrm{CH}_{2} \mathrm{Cl}_{2}$, the organic layer was washed with brine and dried over $\mathrm{Na}_{2} \mathrm{SO}_{4}$. The solvent was removed under reduced pressure to get the crude product, which was used in the next step without further purification.

The resulting residue was dissolved in DCM $(2.0 \mathrm{~mL})$, cooling down to $0{ }^{\circ} \mathrm{C}$, DMAP $(3.0$ $\mathrm{mg}, 0.025 \mathrm{mmol}, 10 \mathrm{~mol} \%)$, TEA $(70 \mathrm{uL}, 0.50 \mathrm{mmol})$ was added to the mixture in $3 \mathrm{~min}$. Finally, benzoyl chloride ( $44 \mathrm{uL}, 0.375 \mathrm{mmol}, 1.5$ eq.) was added dropwise to the flask. The reaction was slowly warmed to room temperature in $2 \mathrm{~min} . \mathrm{K}_{2} \mathrm{CO}_{3}$ aq. was added to the mixture and then the mixture was extracted with $\mathrm{CH}_{2} \mathrm{Cl}_{2}$, the organic layer was washed with brine and dried over $\mathrm{Na}_{2} \mathrm{SO}_{4}$. The solvent was removed under reduced pressure and purified by flash column ( $99 \%$ Hexane/ $\left.1 \% \mathrm{Et}_{2} \mathrm{O}\right)$ to afford the corresponding product $(39.7$ 
mg, 63\% yield over 2 steps). ${ }^{1} \mathrm{H}$ NMR $\left(600 \mathrm{MHz} \mathrm{CDCl}_{3}\right) \delta 8.11(\mathrm{~s}, 1 \mathrm{H}), 7.61(\mathrm{~s}, 1 \mathrm{H}), 7.49$ $(\mathrm{s}, 1 \mathrm{H}), 7.36(\mathrm{~d}, 12 \mathrm{H}, J=7.9 \mathrm{~Hz}), 7.27-7.23(\mathrm{~m}, 27 \mathrm{H}), 4.49-4.40(\mathrm{~m}, 10 \mathrm{H}), 1.49(\mathrm{~d}, 8 \mathrm{H}$, $J=2.3 \mathrm{~Hz}), 1.46-1.44(\mathrm{~m}, 23 \mathrm{H}), 1.17-1.12(\mathrm{~m}, 12 \mathrm{H}) .[\alpha]_{\mathrm{D}}{ }^{20}=-72.3\left(\mathrm{c}=0.45, \mathrm{CHCl}_{3}\right)$, literature ${ }^{6}[\alpha]_{\mathrm{D}}^{20}=-71.6\left(\mathrm{c}=0.96, \mathrm{CHCl}_{3}\right.$, er 95:05).

\section{Reference}

1. Humphries M. J.; Tellmann K. P.; Gibson V. C.; White A. J. P.; Williams D. J. Investigations into the Mechanism of Activation and Initiation of Ethylene Polymerization by Bis(imino)pyridine Cobalt Catalysts: Synthesis, Structures, and Deuterium Labeling Studies. Organometallics 2005, 24, 2039-2050.

2. Guo J.; Shen X.; Lu Z. Regio- and Enantioselective Cobalt-Catalyzed Sequential Hydrosilylation/Hydrogenation of Terminal Alkynes. Angew. Chem. Int. Ed. 2017, 56, 615618.

3. Sherrill W. M.; Kim R.; Rubin M. Improved preparative route toward 3-arylcyclopropenes. Tetrahedron 2008, 64, 8610-8617.

4. Isco M. D.; Wieting J. M.; Mattso A. E. Carbon-Silicon Bond Formation in the Synthesis of Benzylic Silanes. Org. Lett. 2016, 18, 2883-2885.

5. Wang H.; Zhang G.; Zhang Q.; Wang Y.; Li Y.; Xiong, T.; Zhang Q. Copper-Catalyzed NonDirected Hydrosilylation of Cyclopropenes: Highly Diastereoselective Synthesis of Fully Substituted Cyclopropylsilanes. Chem. Commun. 2020, 56, 1819-1822.

6. Parra A.; Amenós L.; Guisán-Ceinos M.; López A.; Garció Ruano J. L.; Tortosa M. CopperCatalyzed Diastereo- and Enantioselective Desymmetrization of Cyclopropenes: Synthesis of Cyclopropylboronates. J. Am. Chem. Soc. 2014, 136, 15833-15836. 


\section{NMR spectrum}

$\mathbf{2 a}, \mathbf{2 b}, \mathbf{2} \mathbf{c}$ and $\mathbf{2 f}$ were already reported in the litereature and our data has been compared and perfectly fit the literature reports. ${ }^{5}$

2a ${ }^{1} \mathrm{H}$ NMR (400 MHz, $\mathrm{CDCl}_{3}$ )

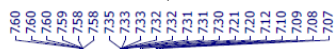

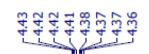

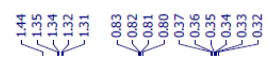
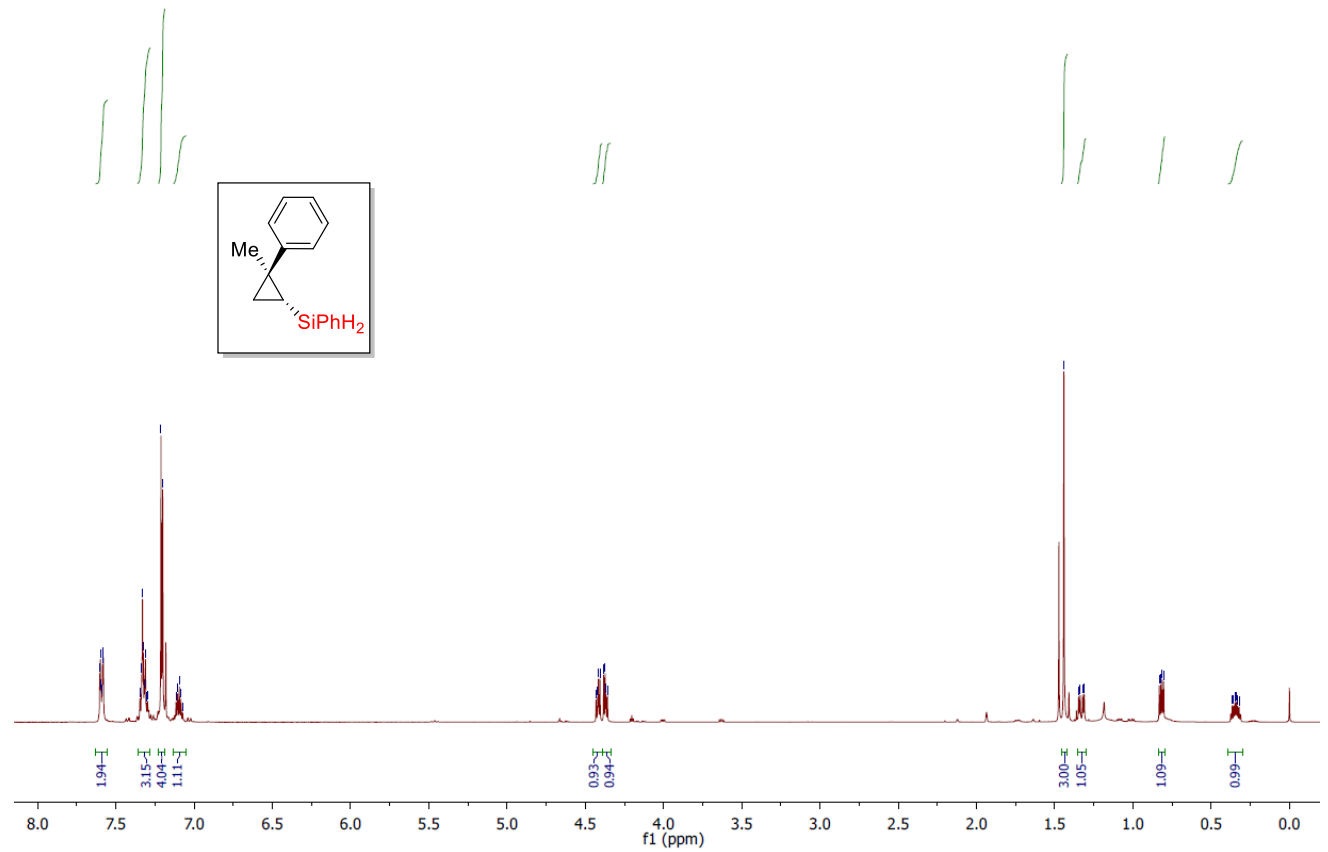

2b ${ }^{1} \mathrm{H}$ NMR (400 MHz, $\left.\mathrm{CDCl}_{3}\right)$
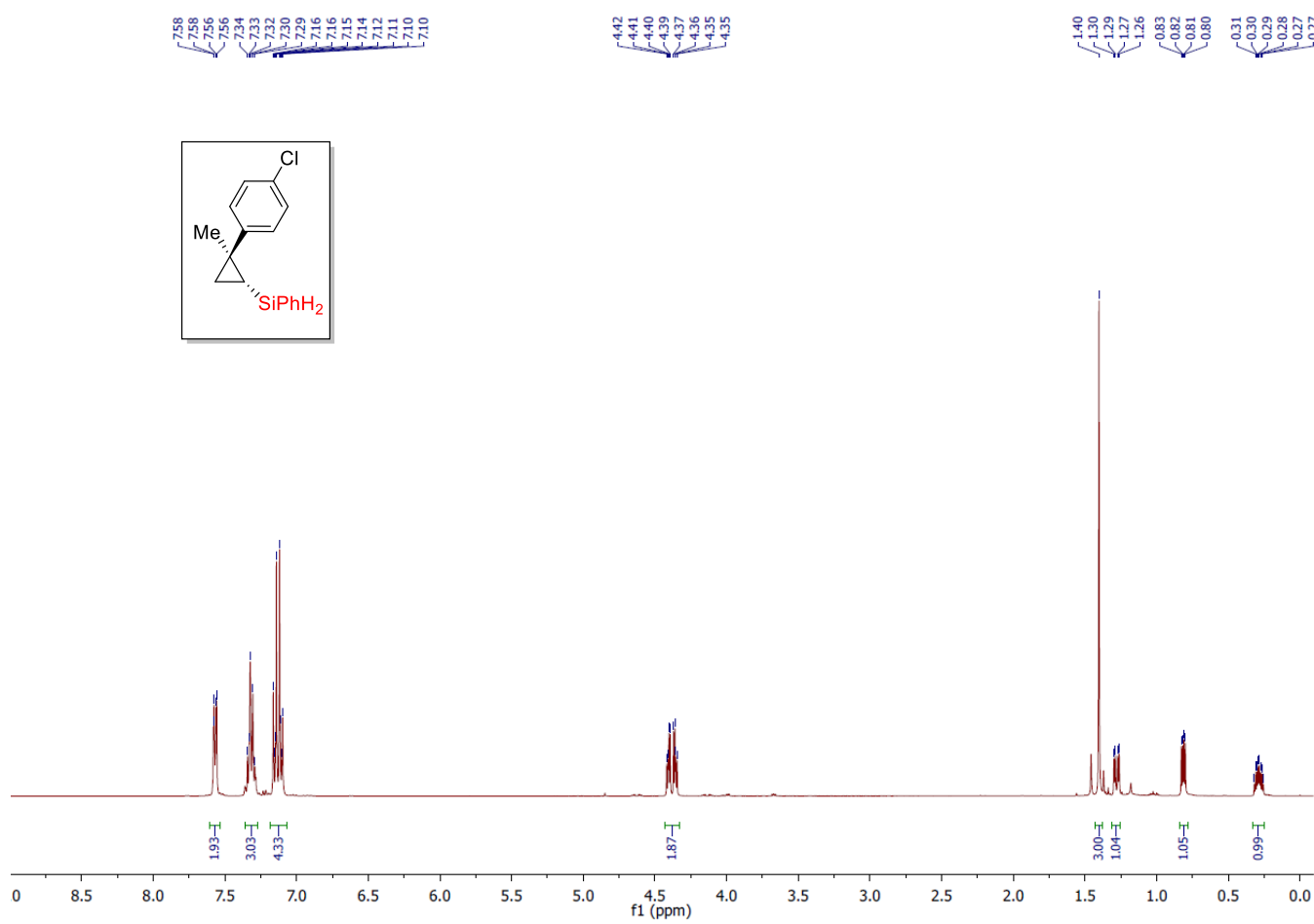
2c ${ }^{1} \mathrm{H}$ NMR (400 MHz, $\mathrm{CDCl}_{3}$ )

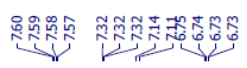
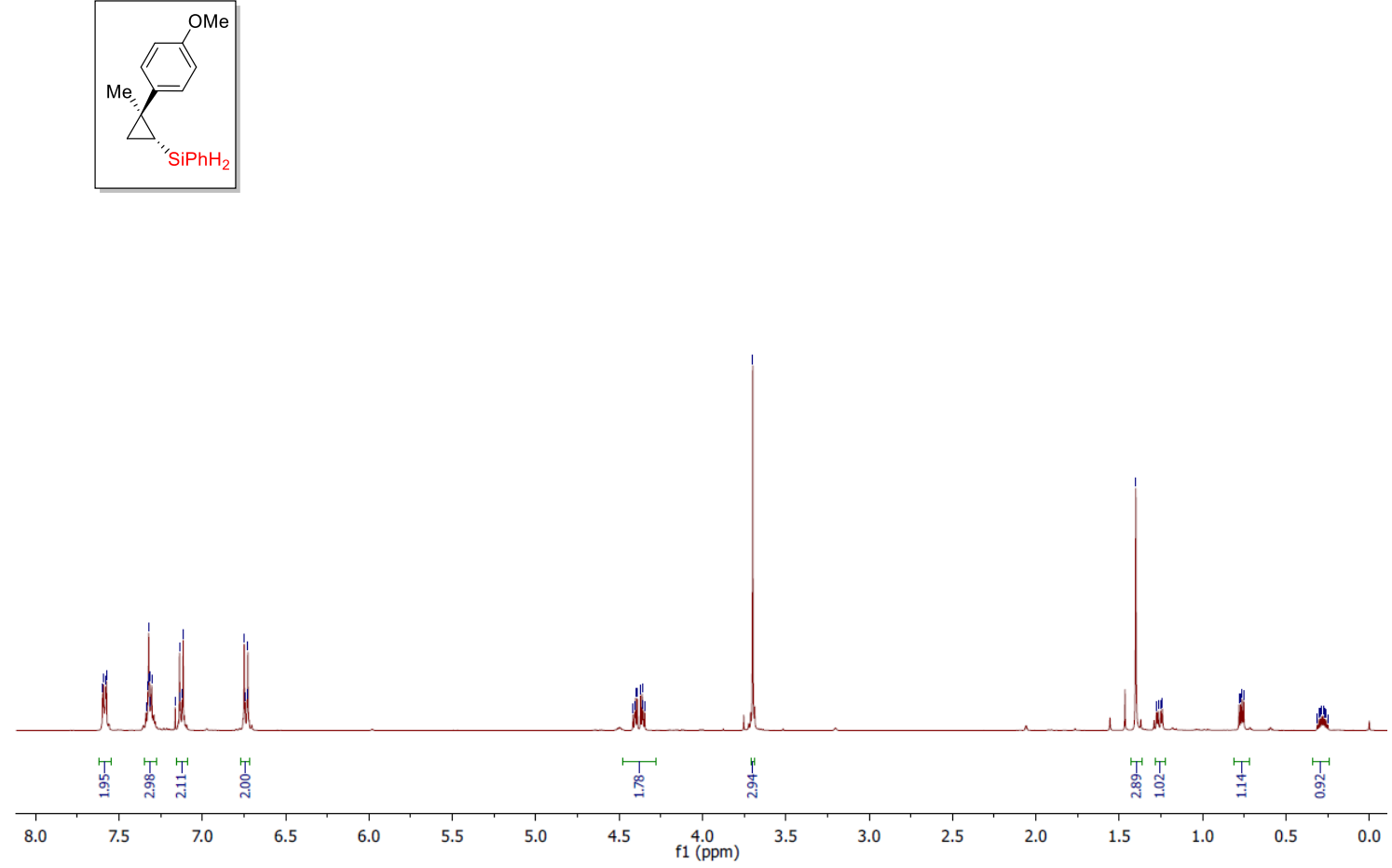

2f ${ }^{1} \mathrm{H}$ NMR (400 MHz, $\mathrm{CDCl}_{3}$ )

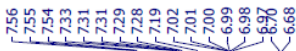
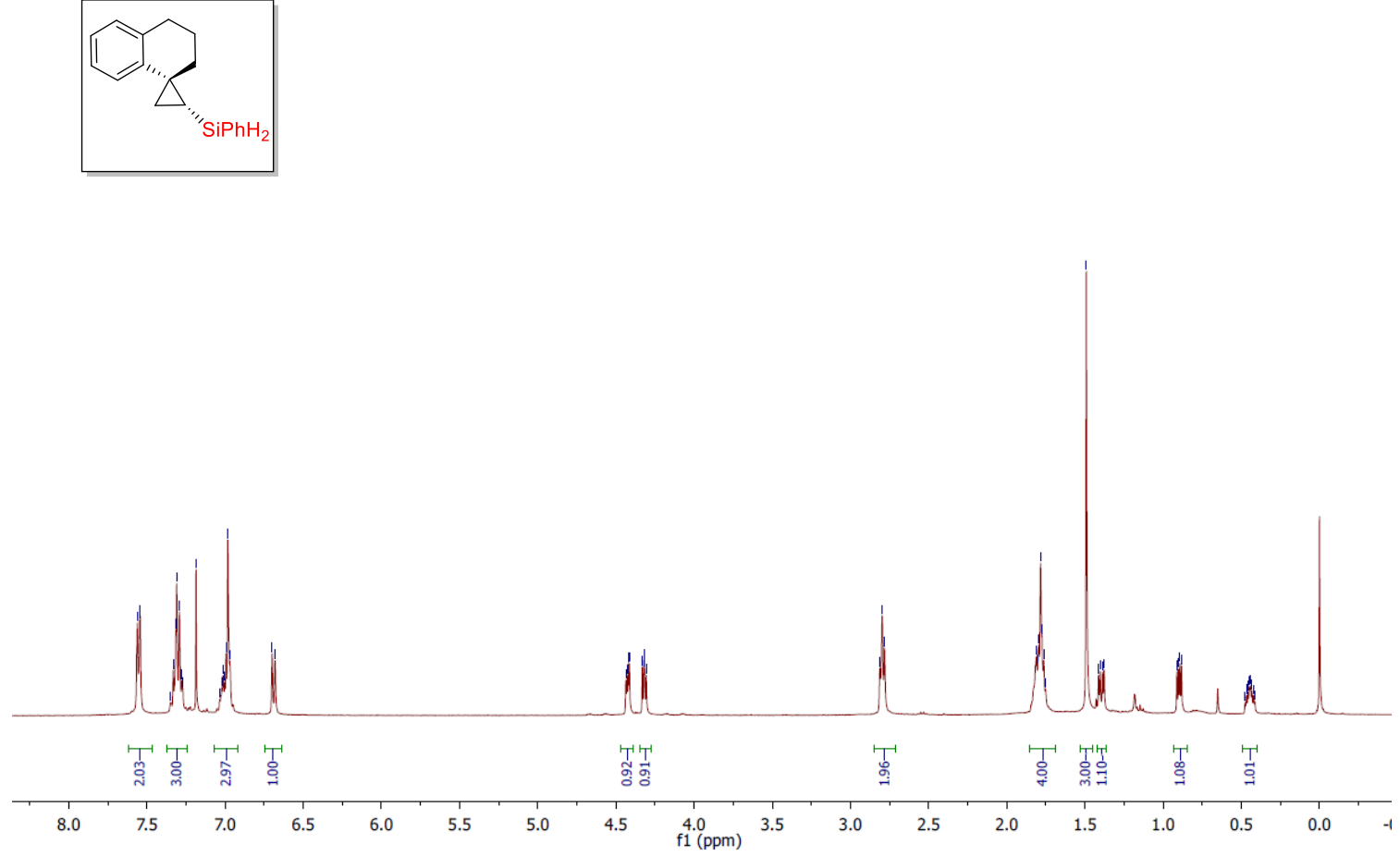
2d

${ }^{1} \mathrm{H}$ NMR (400 $\left.\mathrm{MHz}, \mathrm{CDCl}_{3}\right)$

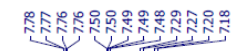
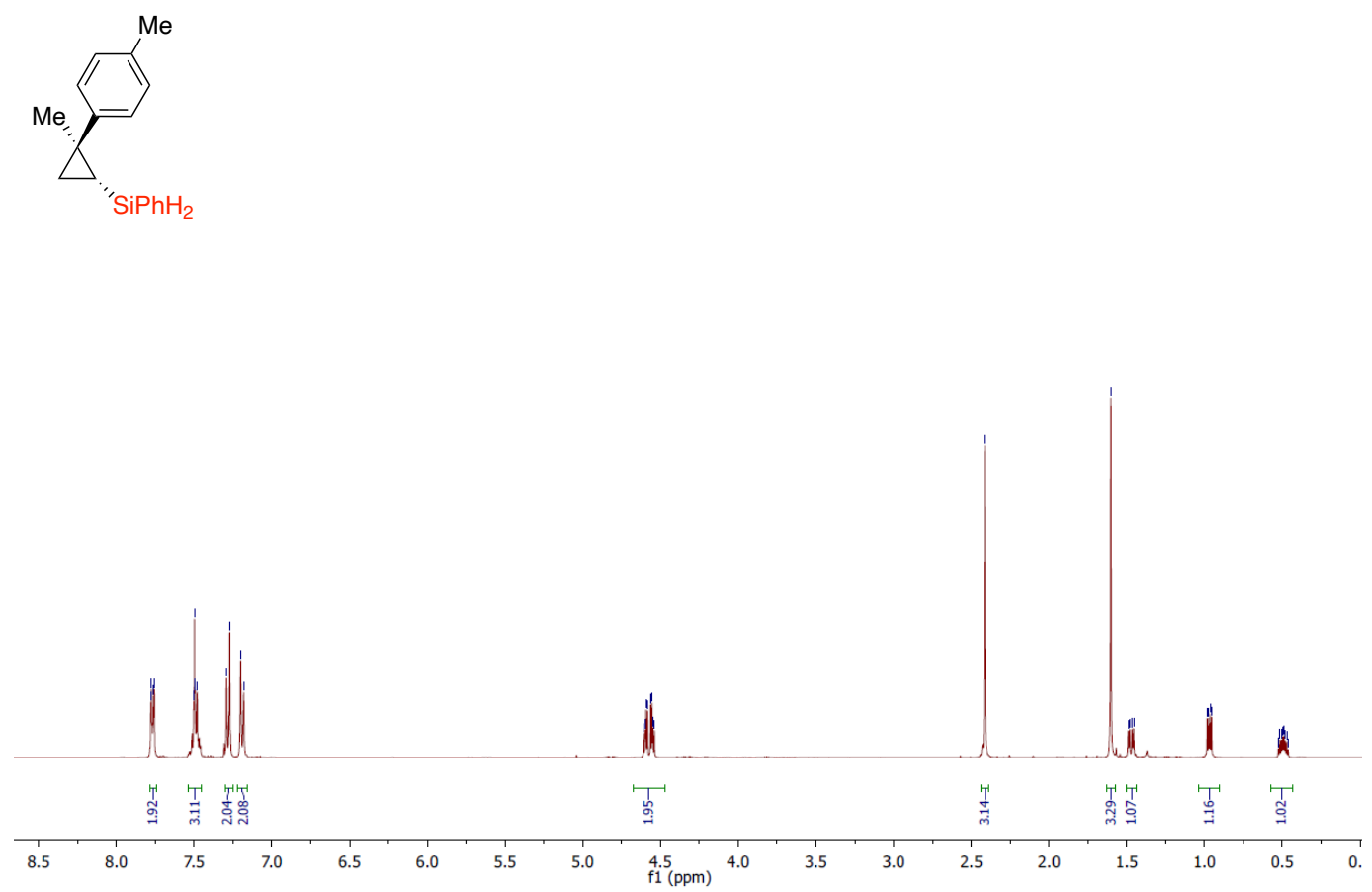

${ }^{13} \mathrm{C}$ NMR (100 MHz, $\left.\mathrm{CDCl}_{3}\right)$
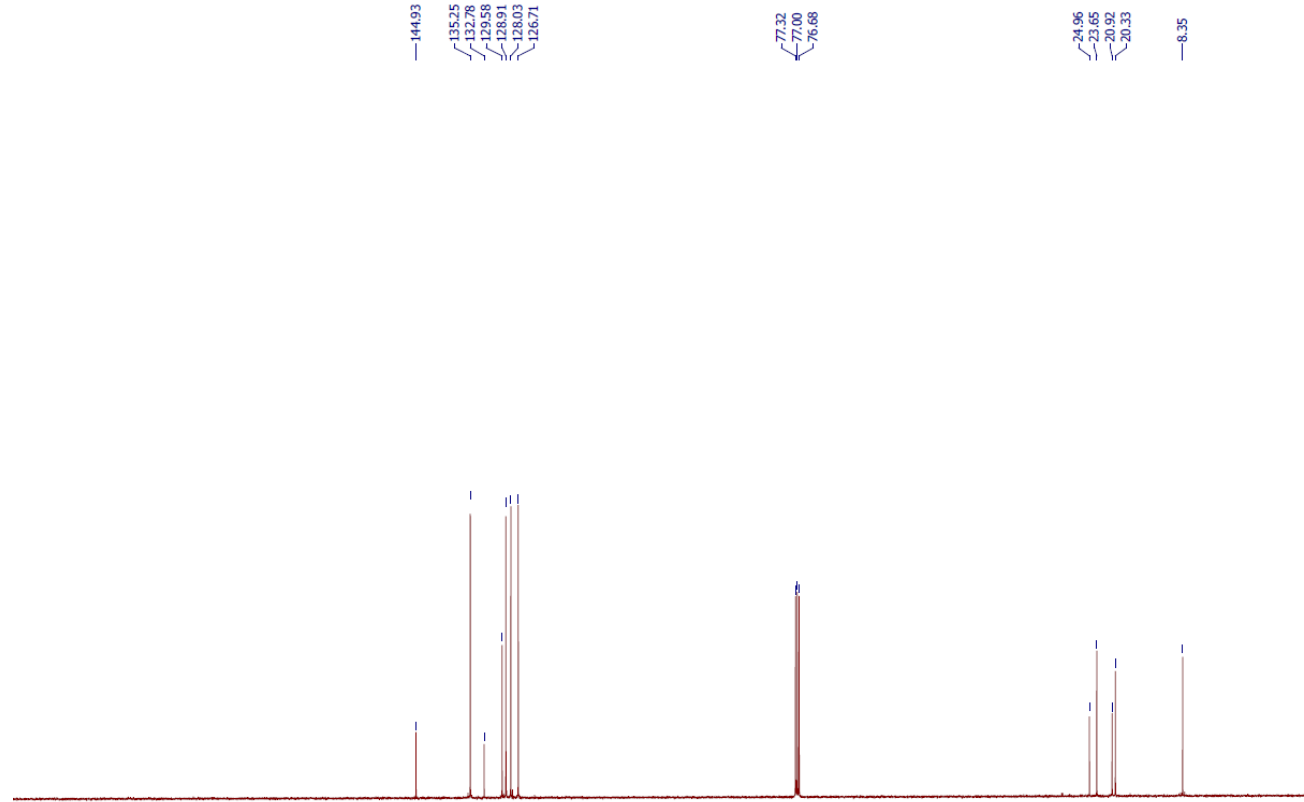

$\begin{array}{lllllllllllllllllllllll}210 & 200 & 190 & 180 & 170 & 160 & 150 & 140 & 130 & 120 & 110 & 100 & 90 & 80 & 70 & 60 & 50 & 40 & 30 & 20 & 10 & 0 & -10\end{array}$ 
2e

${ }^{1} \mathrm{H}$ NMR (400 MHz, $\mathrm{CDCl}_{3}$ )

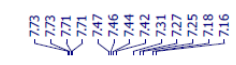
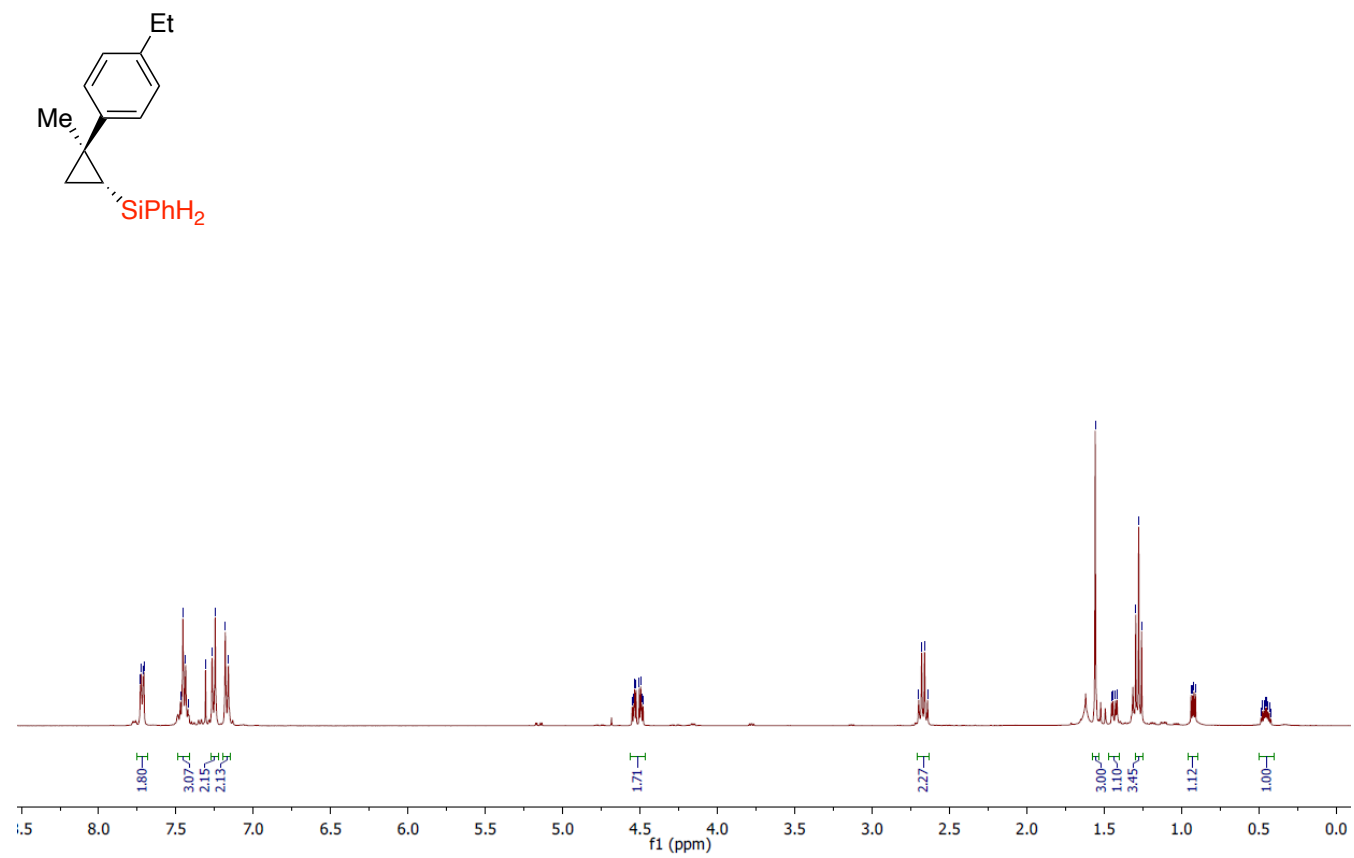

${ }^{13} \mathrm{C}$ NMR (100 MHz, $\left.\mathrm{CDCl}_{3}\right)$
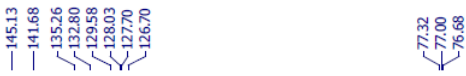

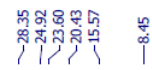

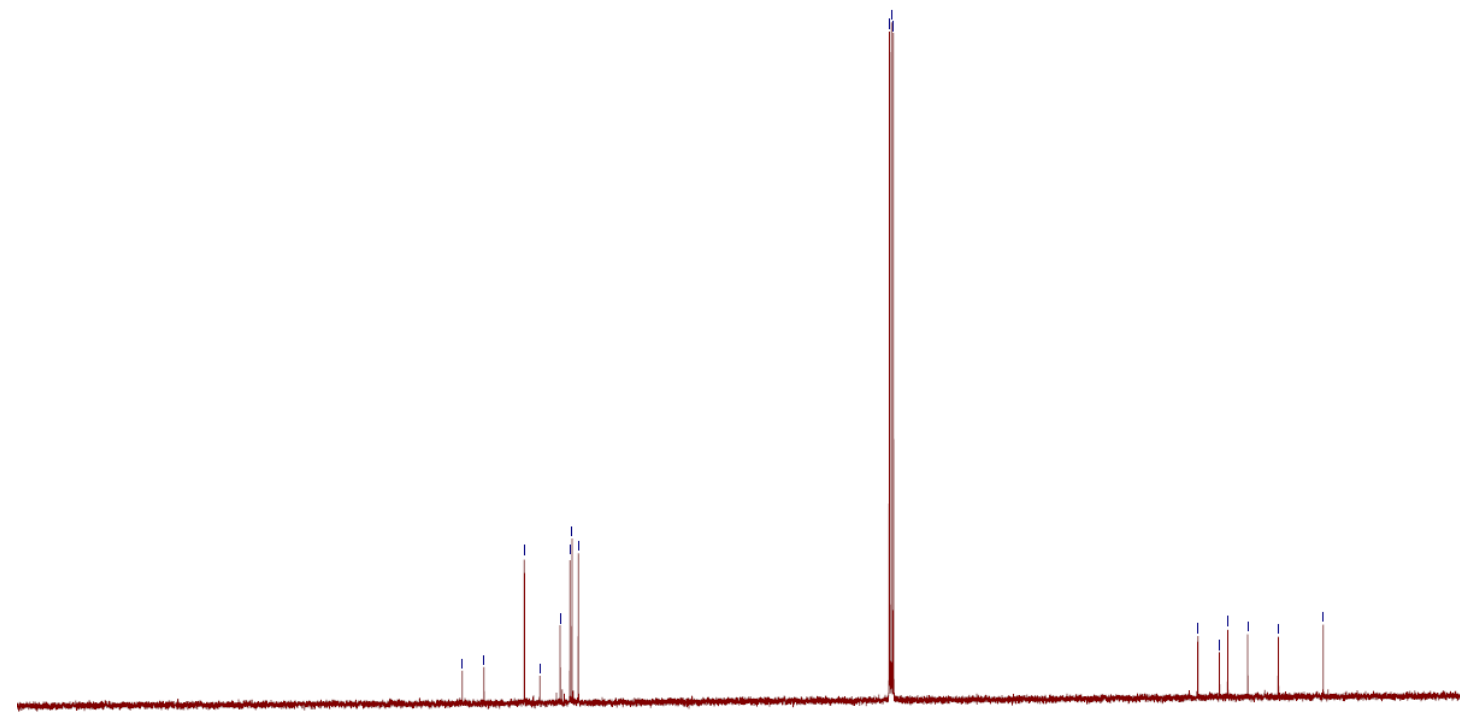

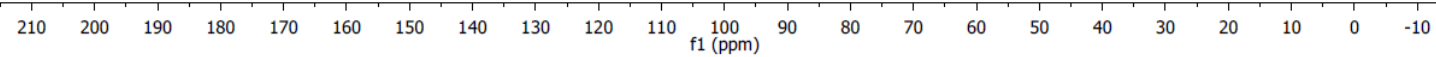


${ }^{1} \mathrm{H}$ NMR (400 MHz, $\mathrm{CDCl}_{3}$ )

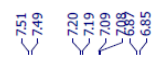
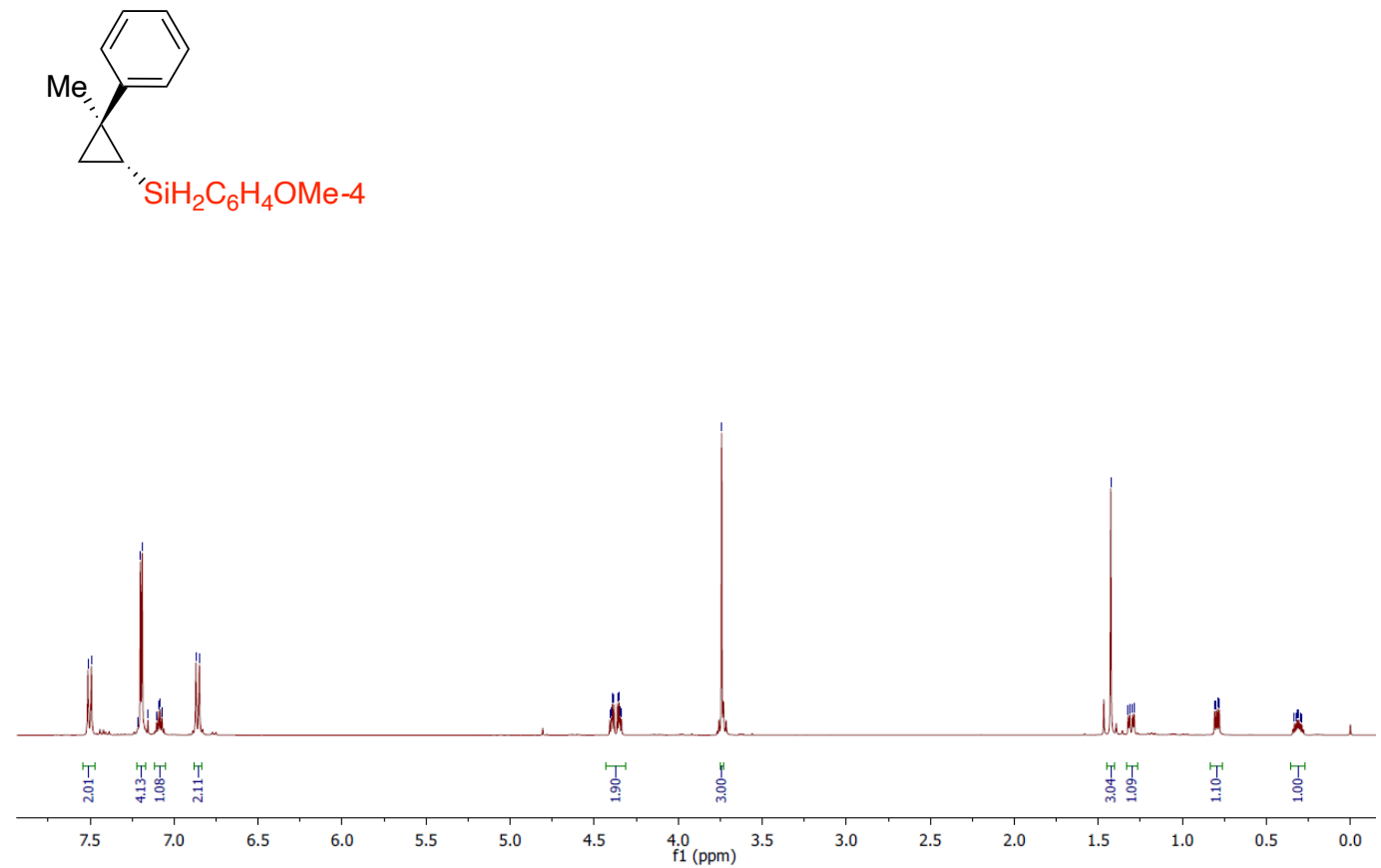

${ }^{13} \mathrm{C}$ NMR (100 MHz, $\left.\mathrm{CDCl}_{3}\right)$
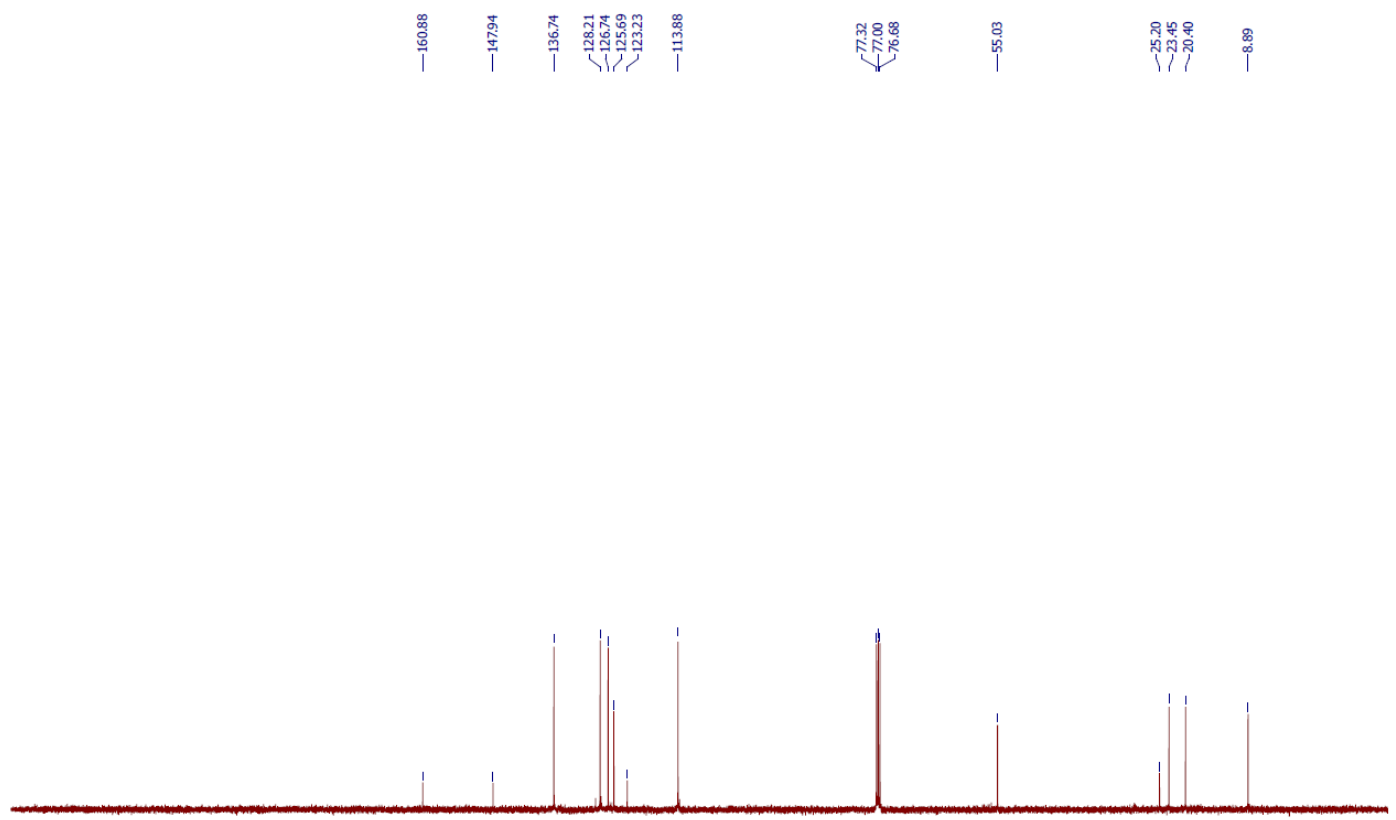

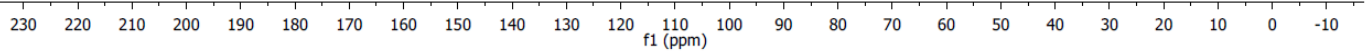


$2 \mathrm{~h}$

${ }^{1} \mathrm{H}$ NMR (400 MHz, $\mathrm{CDCl}_{3}$ )

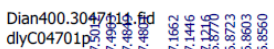

dlyC04701p

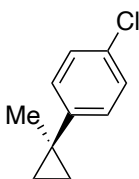

"SiH $\mathrm{C}_{6} \mathrm{H}_{4} \mathrm{OMe}-4$

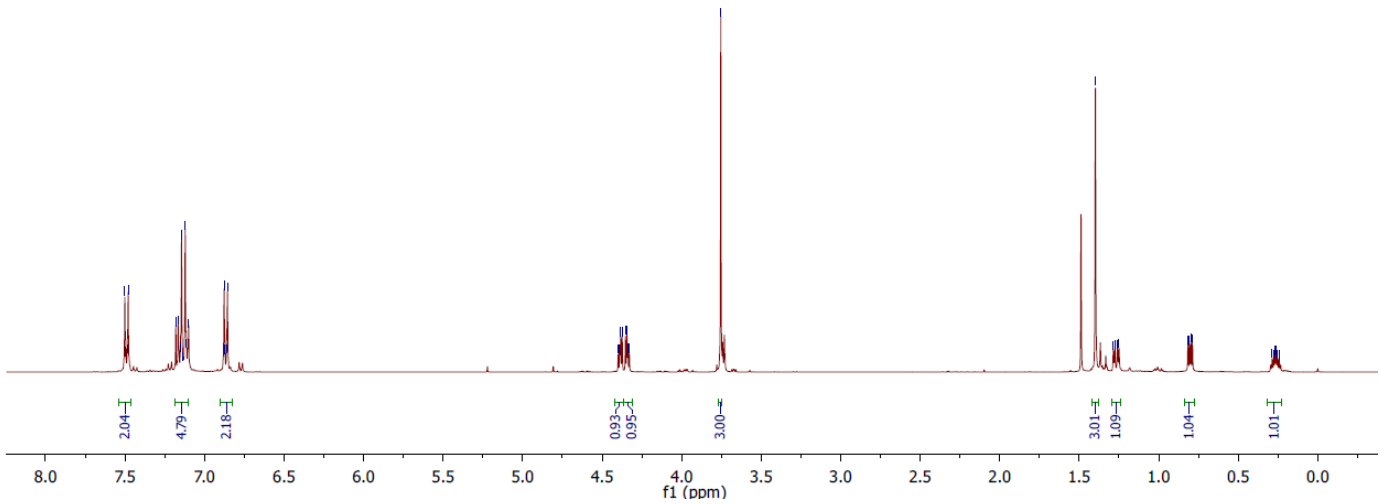

${ }^{13} \mathrm{C}$ NMR (100 MHz, $\left.\mathrm{CDCl}_{3}\right)$

Dian400.3047113.fic dlyC04701p

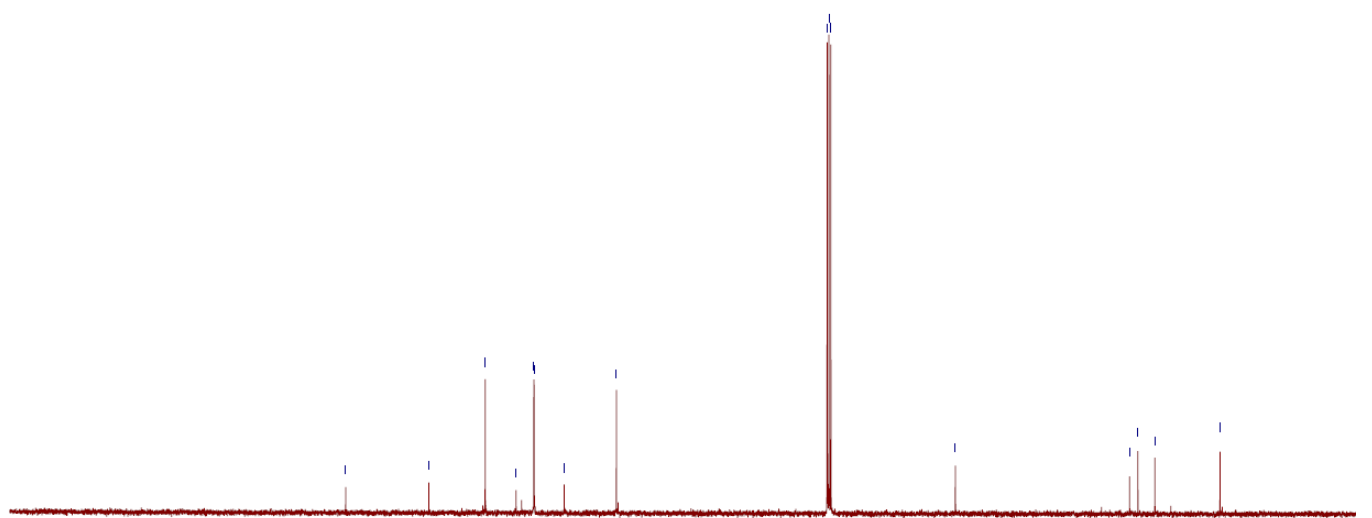

$\begin{array}{llllllllllllllllllllllllllll}210 & 200 & 190 & 180 & 170 & 160 & 150 & 140 & 130 & 120 & 110 & 100 & 90 & 80 & 70 & 60 & 50 & 40 & 30 & 20 & 10 & 0 & -10\end{array}$ 
$2 \mathbf{i}$

${ }^{1} \mathrm{H}$ NMR (400 $\mathrm{MHz}, \mathrm{CDCl}_{3}$ )

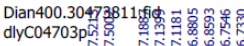

dlyC04703po

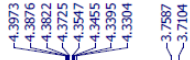

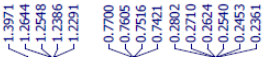<smiles>COc1ccc(C2(C)CC2)cc1</smiles>

" $\mathrm{SiH}_{2} \mathrm{C}_{6} \mathrm{H}_{4} \mathrm{OMe}-4$

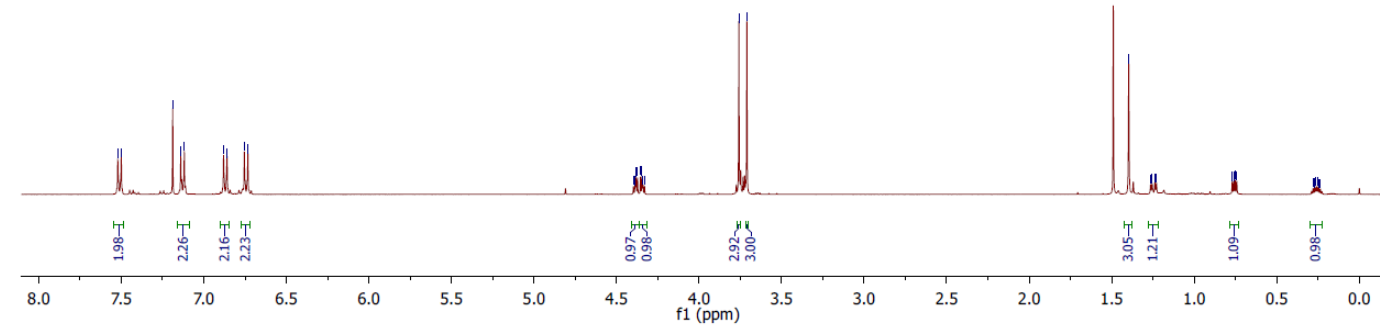

${ }^{13} \mathrm{C}$ NMR (100 MHz, $\left.\mathrm{CDCl}_{3}\right)$

Dian400.30473812.fid

dlyc04703p

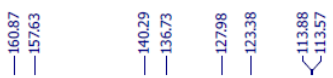

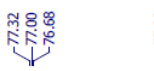

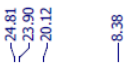

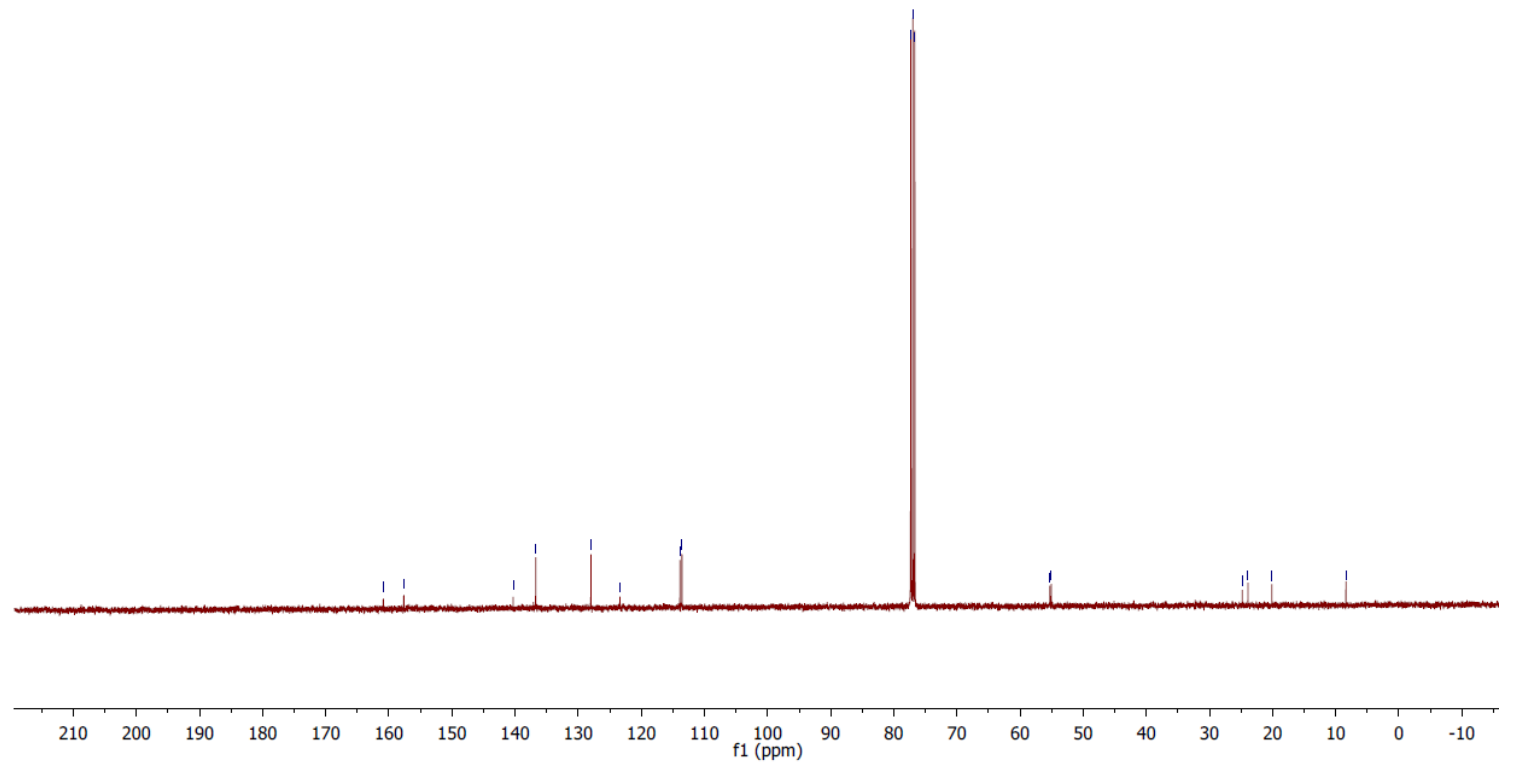


2j

${ }^{1} \mathrm{H}$ NMR (400 MHz, $\mathrm{CDCl}_{3}$ )

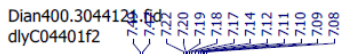

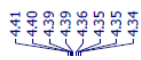

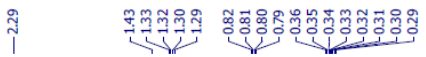

$\mathrm{Me}=$

"' $\mathrm{SiH}_{2} \mathrm{C}_{6} \mathrm{H}_{4} \mathrm{Me}-4$

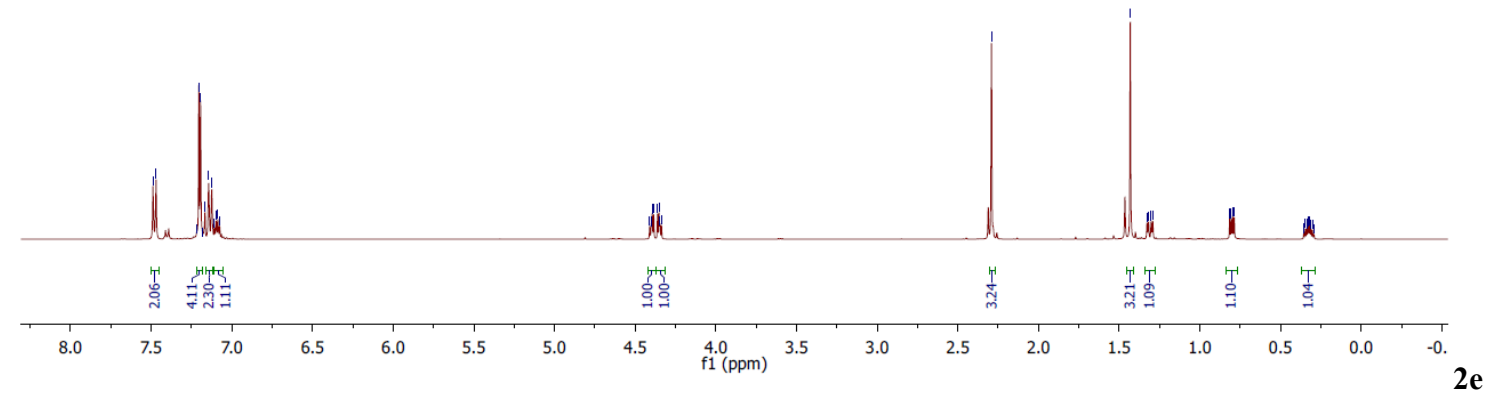

${ }^{13} \mathrm{C}$ NMR (100 MHz, $\left.\mathrm{CDCl}_{3}\right)$

Dian400.3044123.fid dlyC04401f2

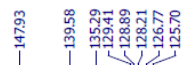

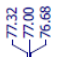

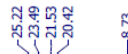

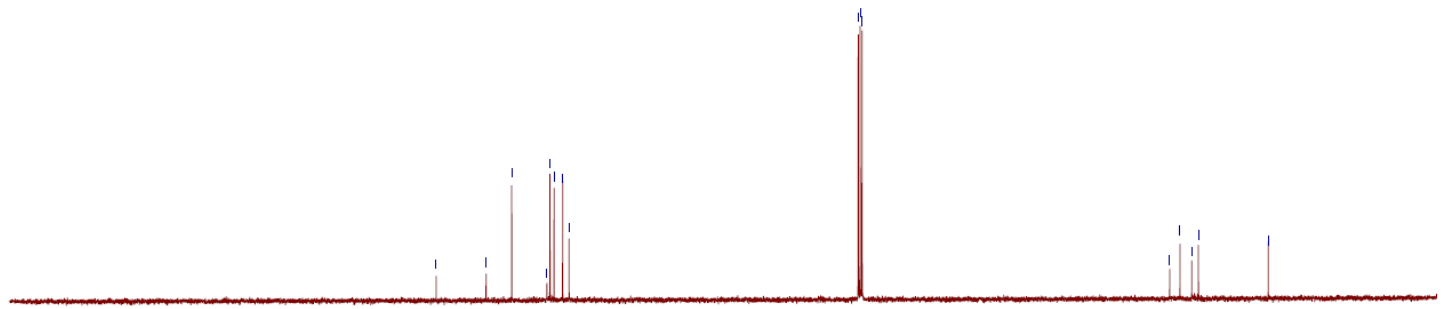

$\begin{array}{lllllllllllllllllllllll}210 & 200 & 190 & 180 & 170 & 160 & 150 & 140 & 130 & 120 & 110 & 100 & 90 & 80 & 70 & 60 & 50 & 40 & 30 & 20 & 10 & 0 & -10\end{array}$ 
2k

${ }^{1} \mathrm{H}$ NMR (400 $\mathrm{MHz}, \mathrm{CDCl}_{3}$ )

Dian400
dlyC04403p pure 4CIC6H4SiH3PhMeCP

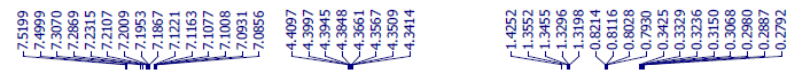

$\mathrm{Me}=$

" $\mathrm{SiH}_{2} \mathrm{C}_{6} \mathrm{H}_{4} \mathrm{Cl}-4$

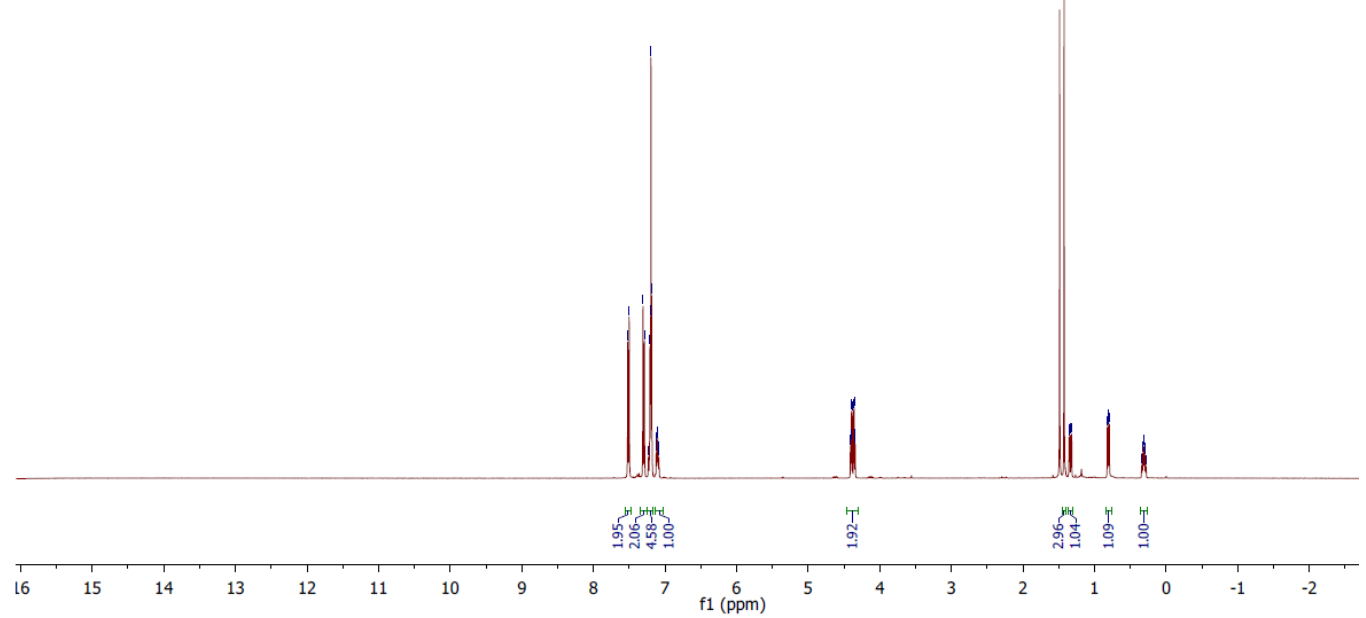

${ }^{13} \mathrm{C}$ NMR (100 MHz, $\left.\mathrm{CDCl}_{3}\right)$

Dian400

dlyC04403p pure 4CIC6H4SiH3PhMeCP

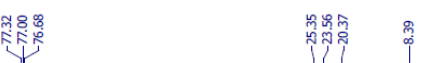

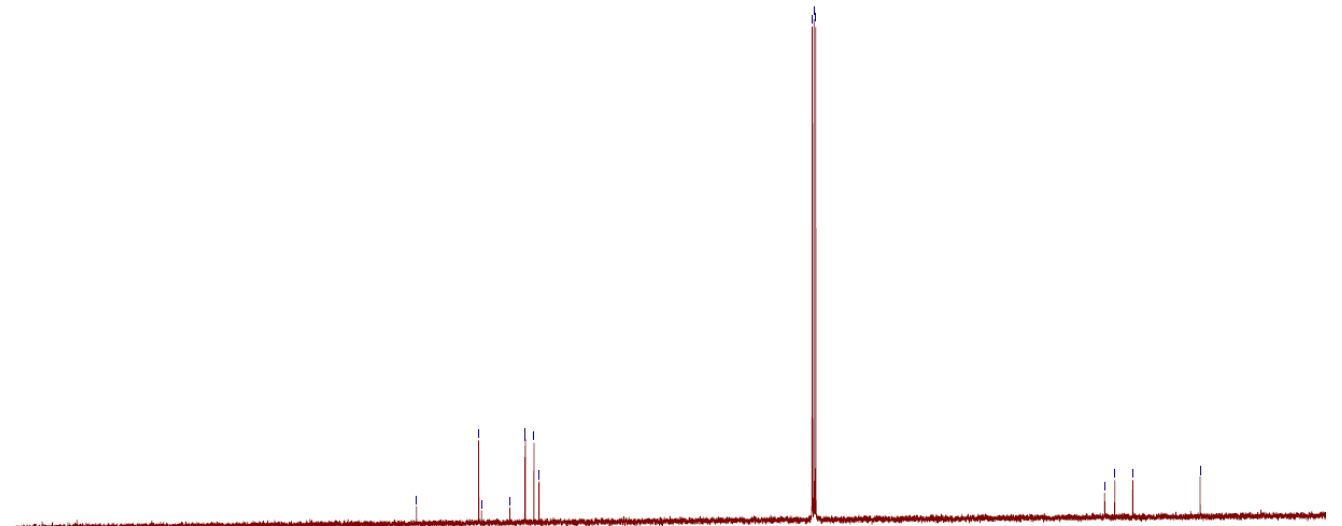

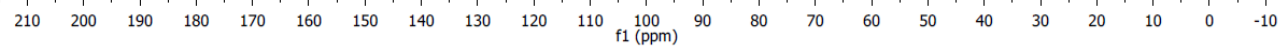


21

${ }^{1} \mathrm{H}$ NMR (400 MHz, $\mathrm{CDCl}_{3}$ )

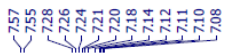

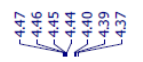<smiles>CC1(c2ccccc2)CC1</smiles>

" $\mathrm{SiH}_{2} \mathrm{C}_{6} \mathrm{H}_{4} \mathrm{Me}-2$

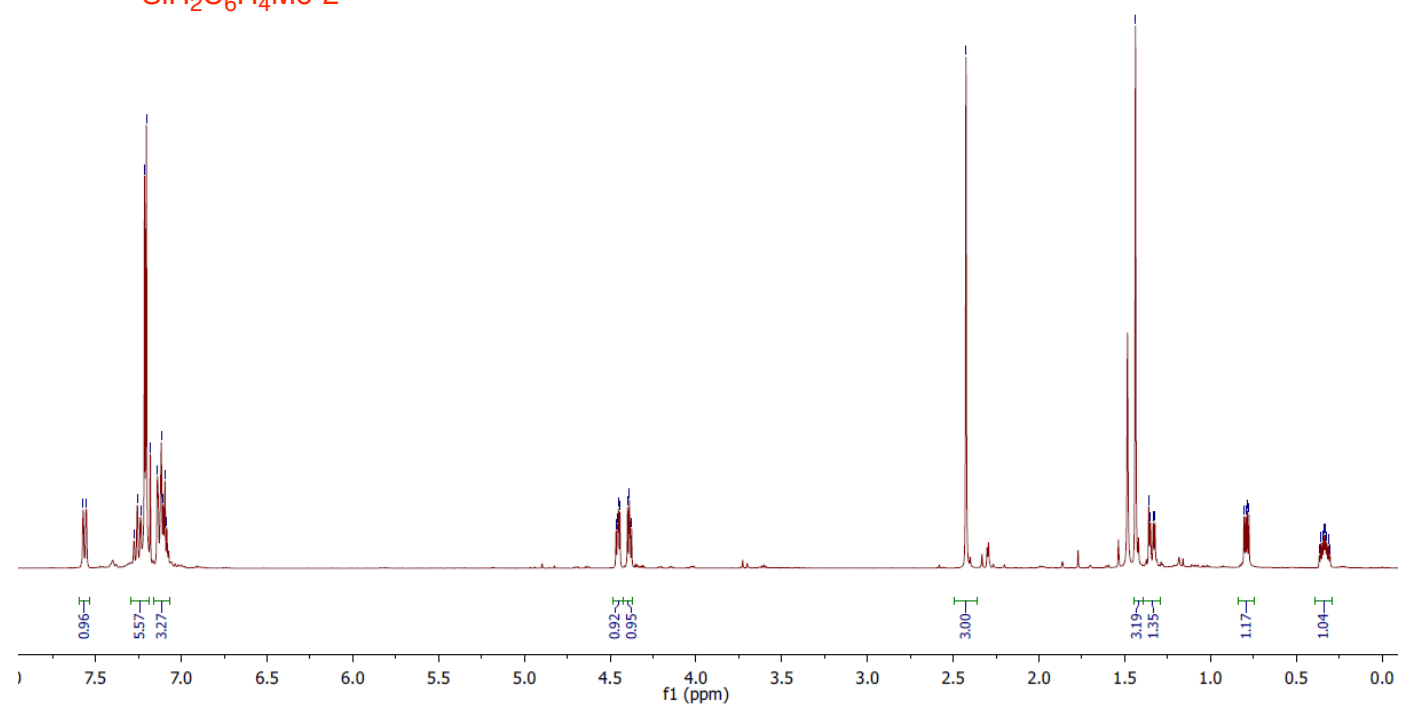

${ }^{13} \mathrm{C}$ NMR (100 MHz, $\left.\mathrm{CDCl}_{3}\right)$

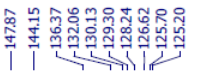

象禺
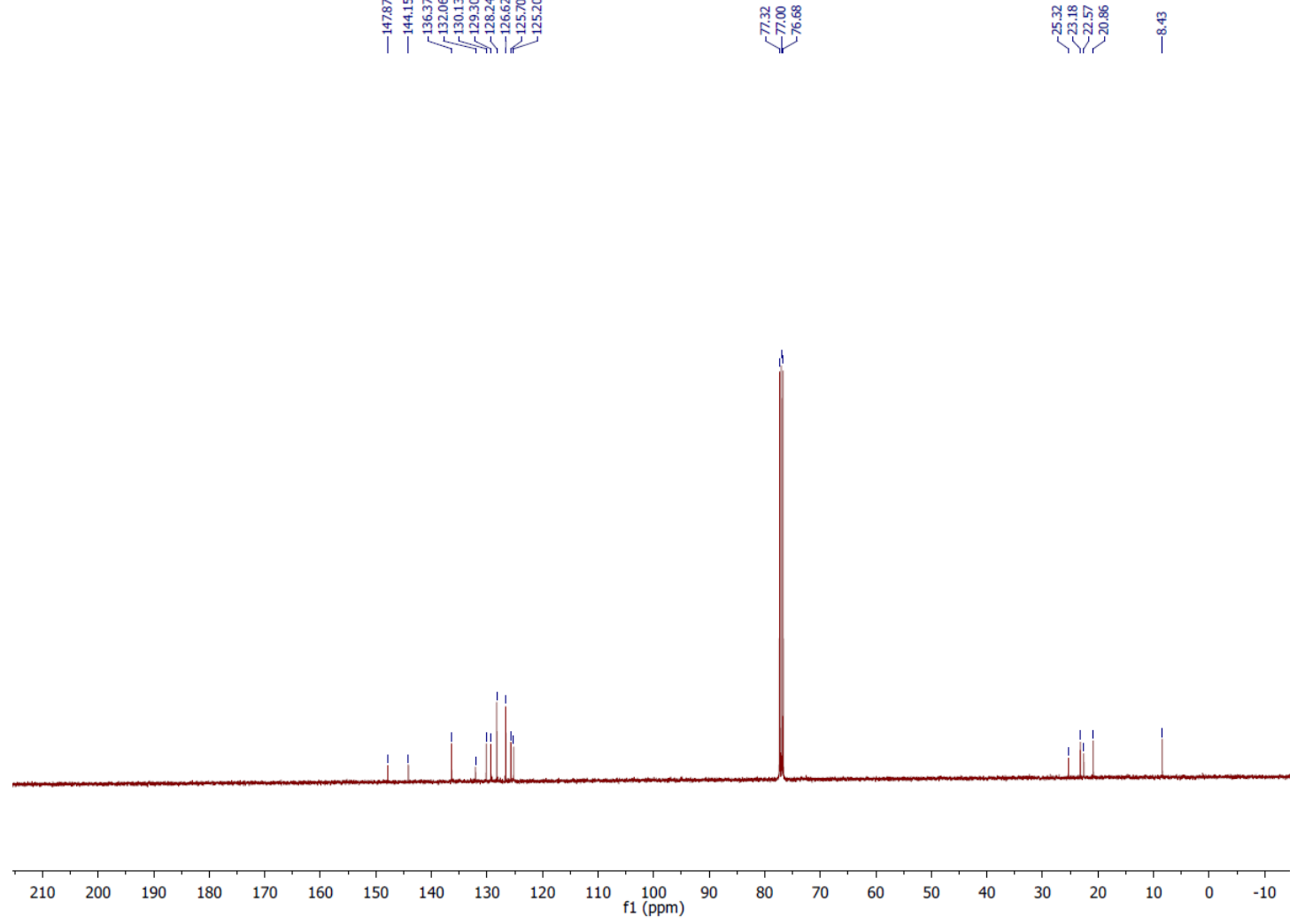
$2 \mathrm{~m}$

${ }^{1} \mathrm{H}$ NMR (400 MHz, $\mathrm{CDCl}_{3}$ )

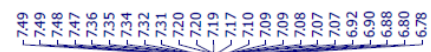

$\mathrm{Me}=$

" $\mathrm{SiH}_{2} \mathrm{C}_{6} \mathrm{H}_{4} \mathrm{OMe}-2$

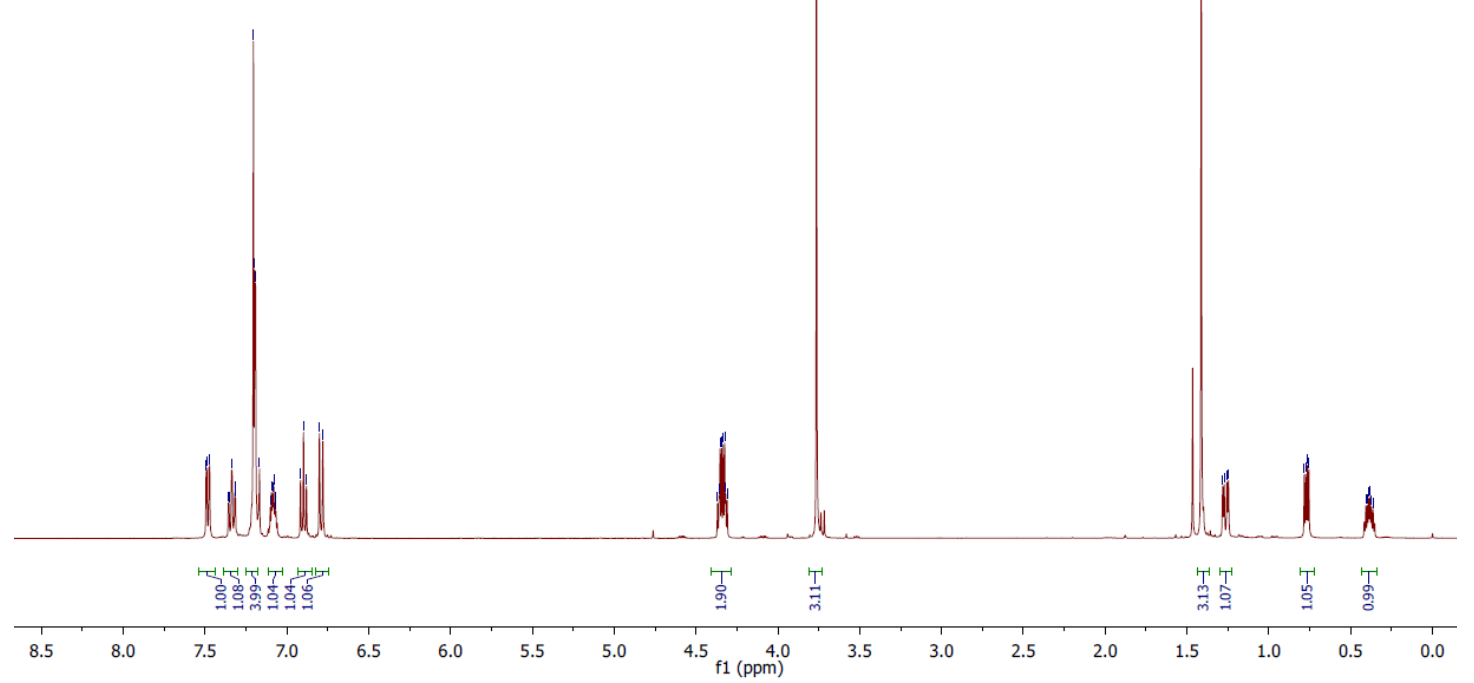

${ }^{13} \mathrm{C}$ NMR (100 MHz, $\left.\mathrm{CDCl}_{3}\right)$
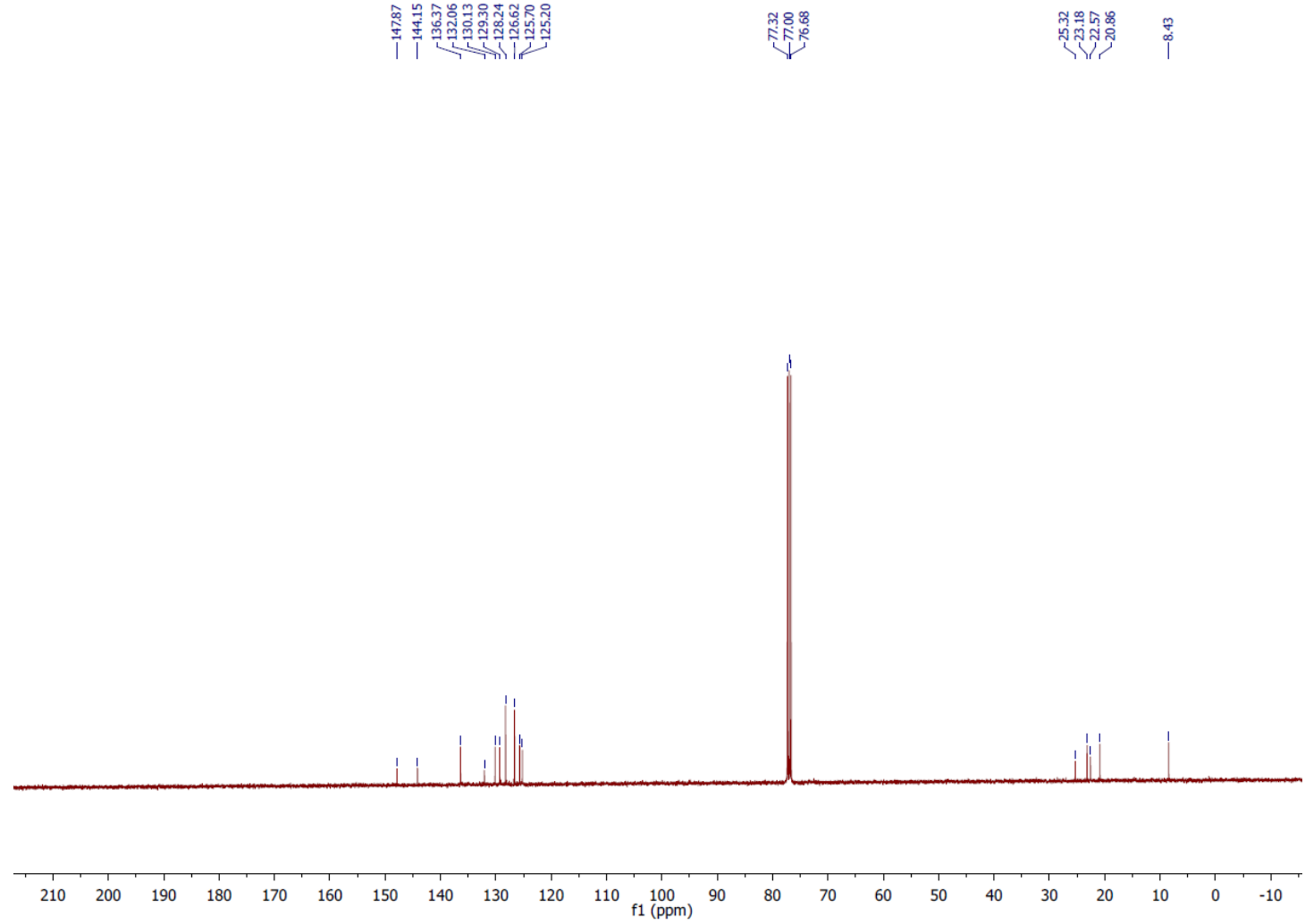
2n

${ }^{1} \mathrm{H}$ NMR (400 MHz, $\left.\mathrm{CDCl}_{3}\right)$

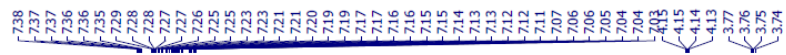

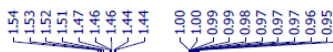
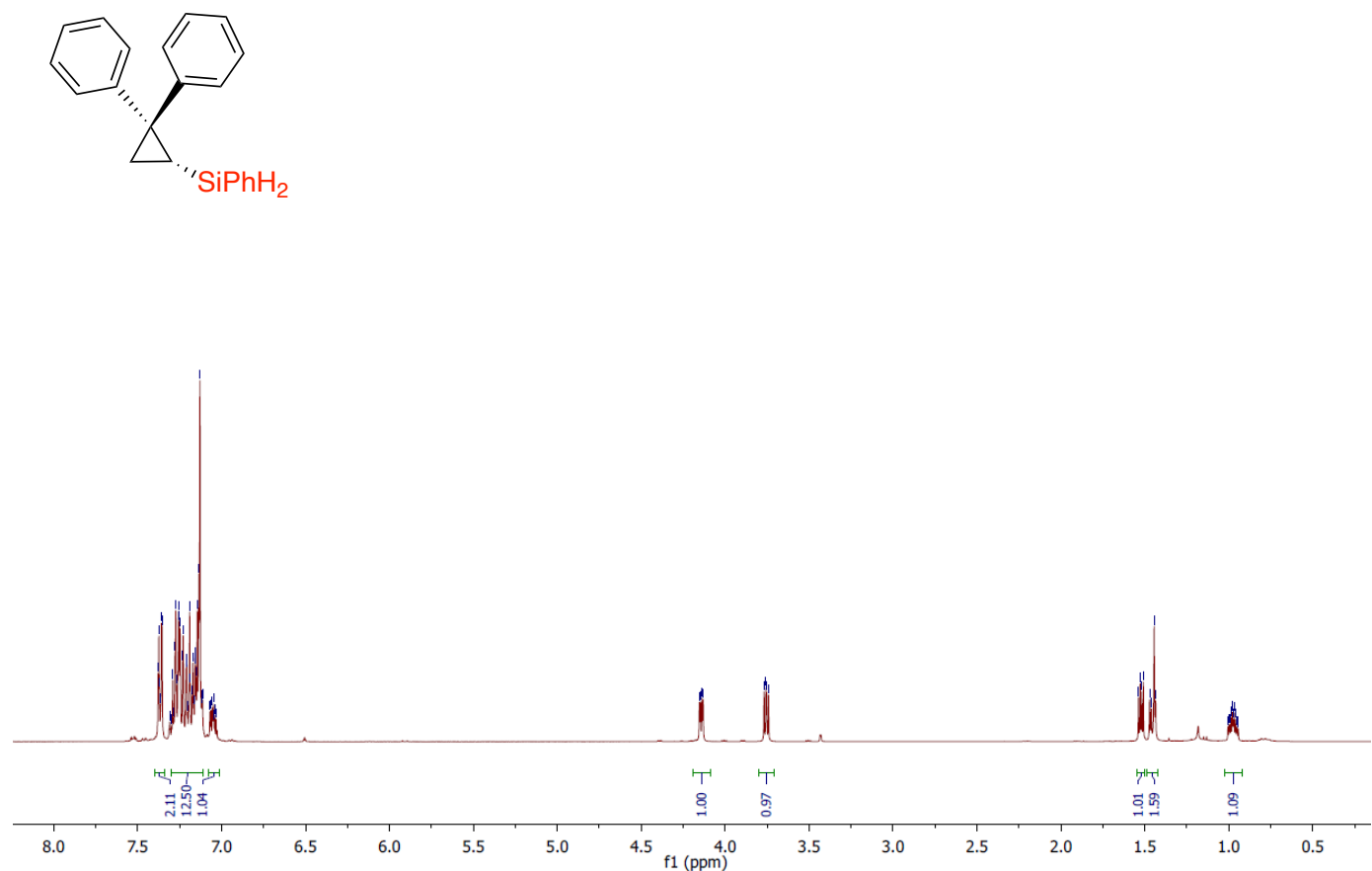

${ }^{13} \mathrm{C}$ NMR (100 MHz, $\left.\mathrm{CDCl}_{3}\right)$

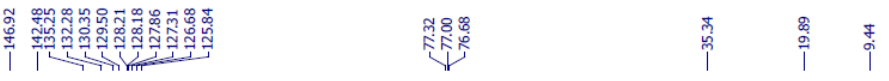

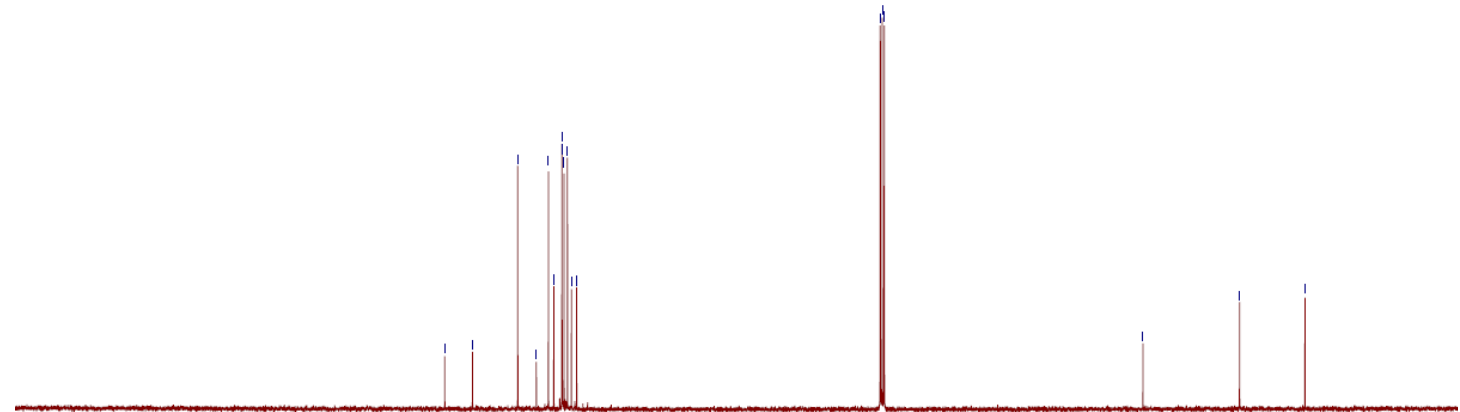

$\begin{array}{lllllllllllllllllllllll}210 & 200 & 190 & 180 & 170 & 160 & 150 & 140 & 130 & 120 & 110 & 100 & 90 & 80 & 70 & 60 & 50 & 40 & 30 & 20 & 10 & 0 & -10\end{array}$ 
20

${ }^{1} \mathrm{H}$ NMR (400 MHz, $\mathrm{CDCl}_{3}$ )

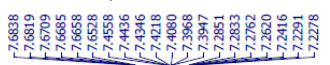

高亭

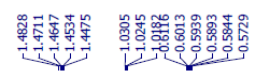
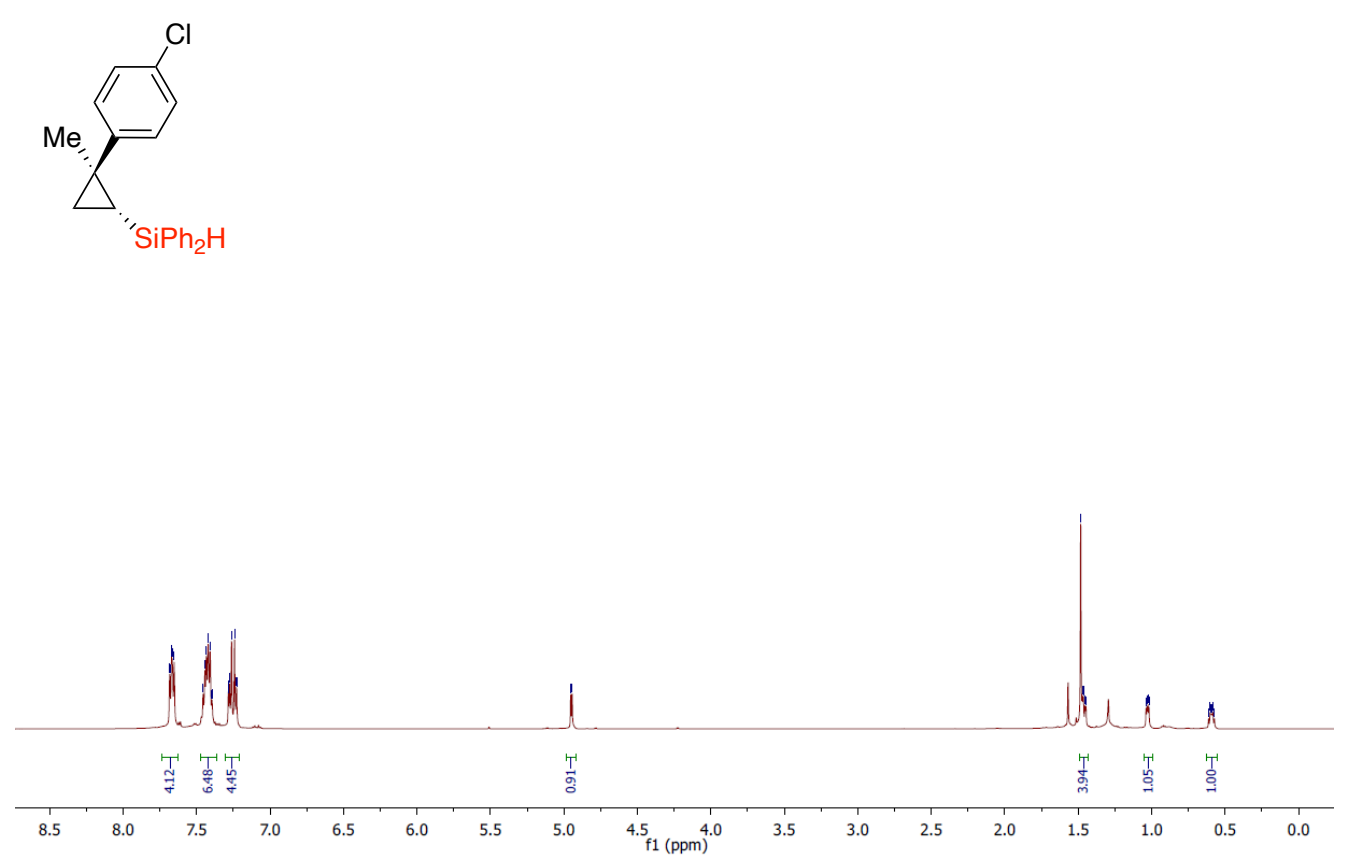

${ }^{13} \mathrm{C}$ NMR (100 MHz, $\left.\mathrm{CDCl}_{3}\right)$

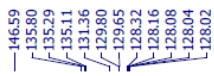

ำ

ฟึํํำ
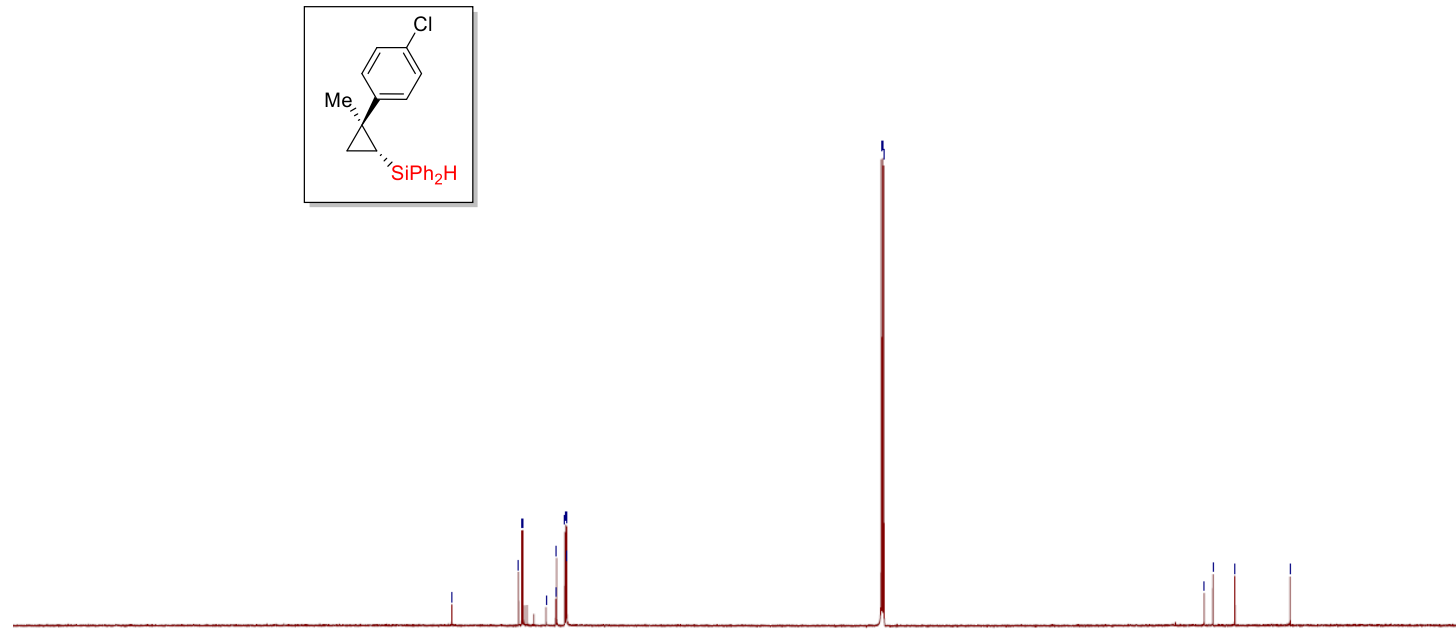

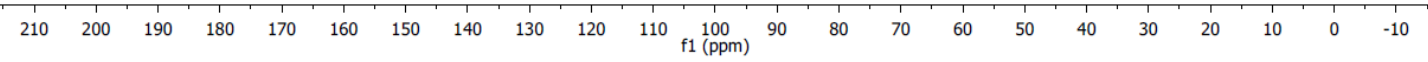


$2 \mathbf{p}$

${ }^{1} \mathrm{H}$ NMR (400 MHz, $\left.\mathrm{CDCl}_{3}\right)$
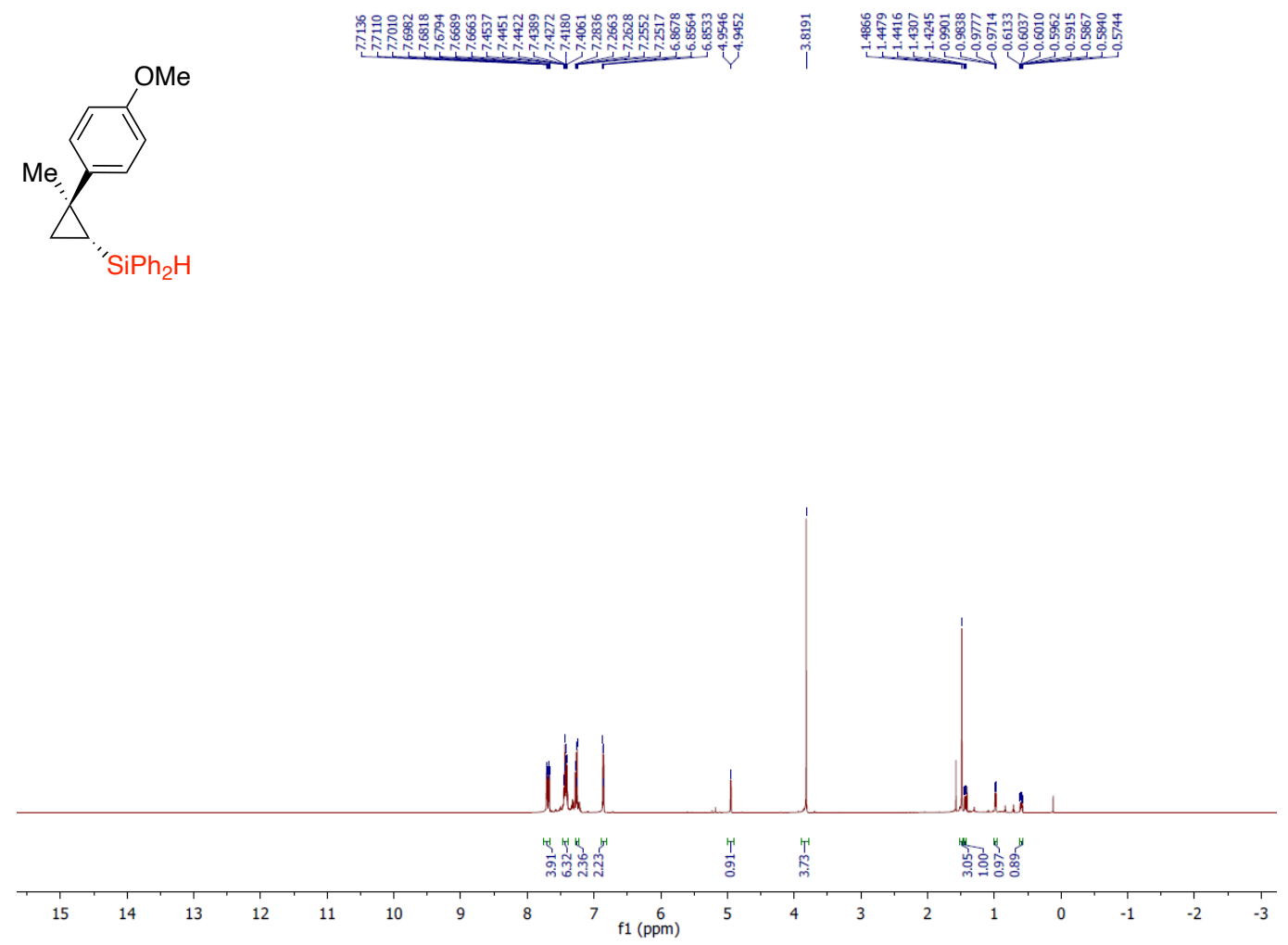

${ }^{13} \mathrm{C}$ NMR (100 MHz, $\left.\mathrm{CDCl}_{3}\right)$

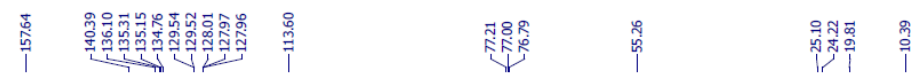

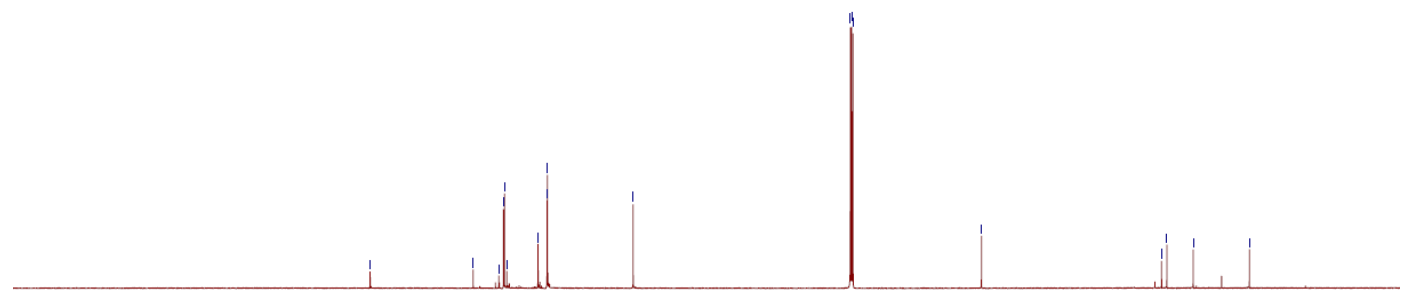

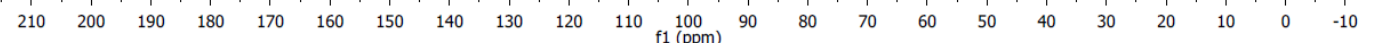


$2 q$

${ }^{1} \mathrm{H}$ NMR (400 MHz, $\mathrm{CDCl}_{3}$ )

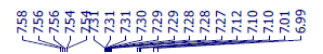
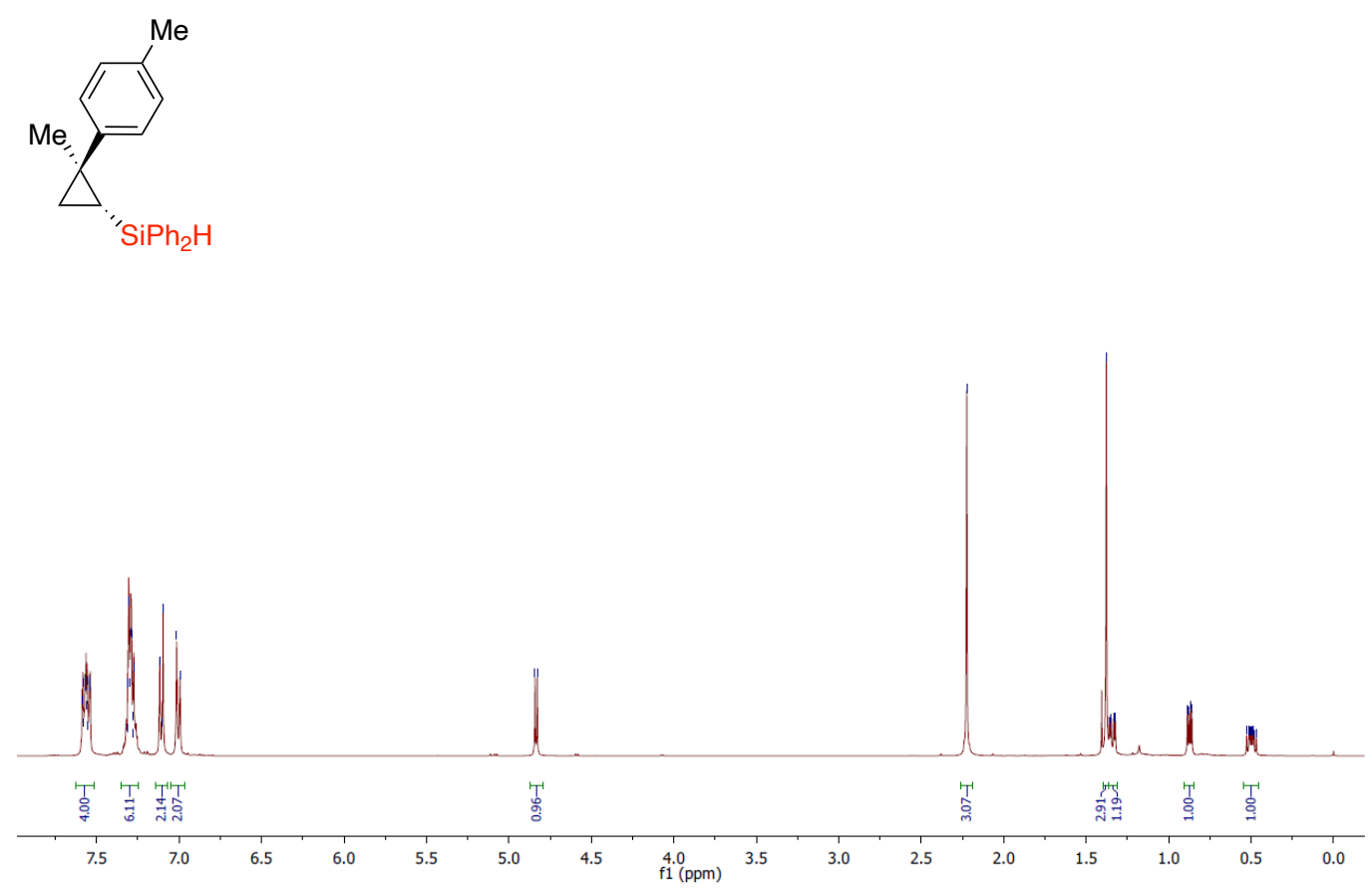

${ }^{13} \mathrm{C}$ NMR (100 MHz, $\left.\mathrm{CDCl}_{3}\right)$
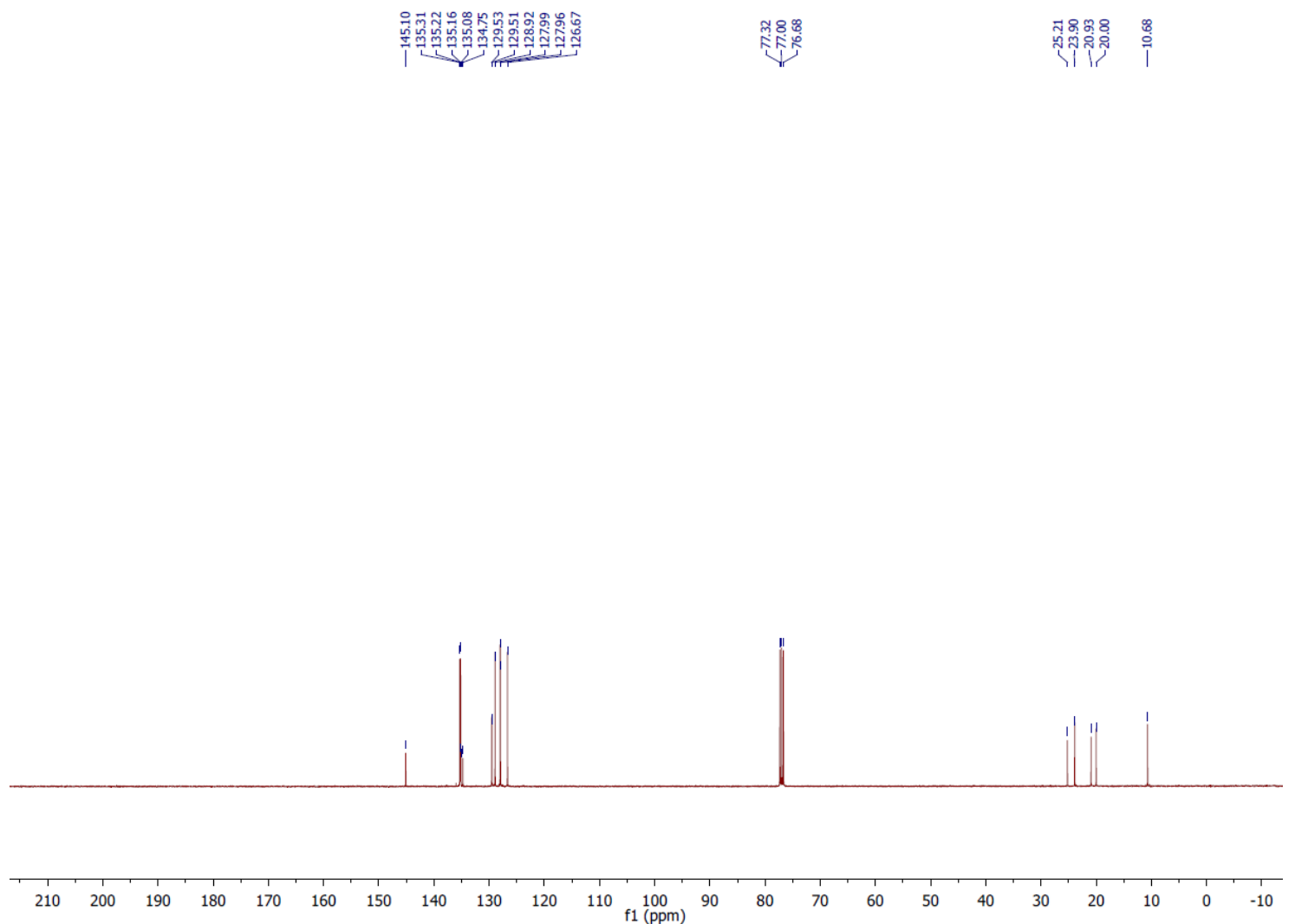
2r

${ }^{1} \mathrm{H}$ NMR (400 MHz, $\mathrm{CDCl}_{3}$ )

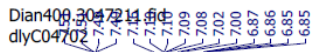

$\mathrm{Me}, \mathrm{Me}$

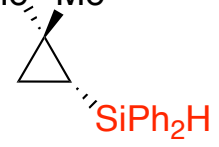

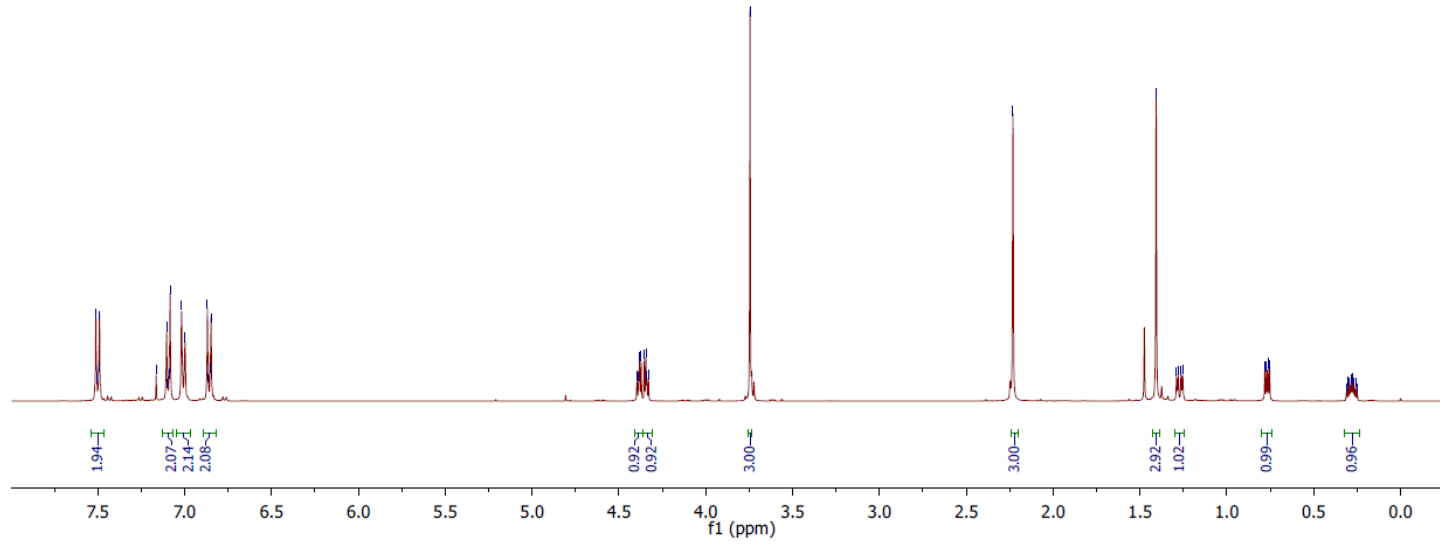

${ }^{13} \mathrm{C}$ NMR (100 MHz, $\left.\mathrm{CDCl}_{3}\right)$

Dian400.3047212.fid dlyc04702

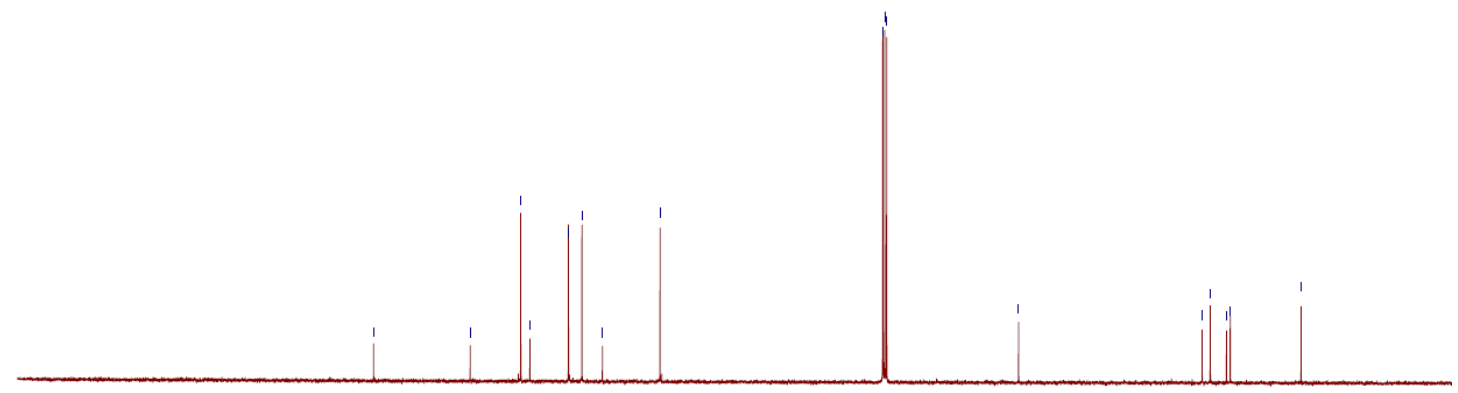

$\begin{array}{lllllllllllllllllllllll}210 & 200 & 190 & 180 & 170 & 160 & 150 & 140 & 130 & 120 & 110 & 100 & 90 & 80 & 70 & 60 & 50 & 40 & 30 & 20 & 10 & 0 & -10\end{array}$ 
2s

${ }^{1} \mathrm{H}$ NMR (400 MHz, $\mathrm{CDCl}_{3}$ )

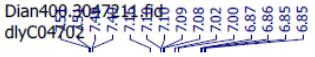

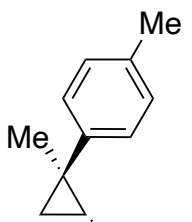

" $\mathrm{SiH}_{2} \mathrm{C}_{6} \mathrm{H}_{4} \mathrm{pOMe}$

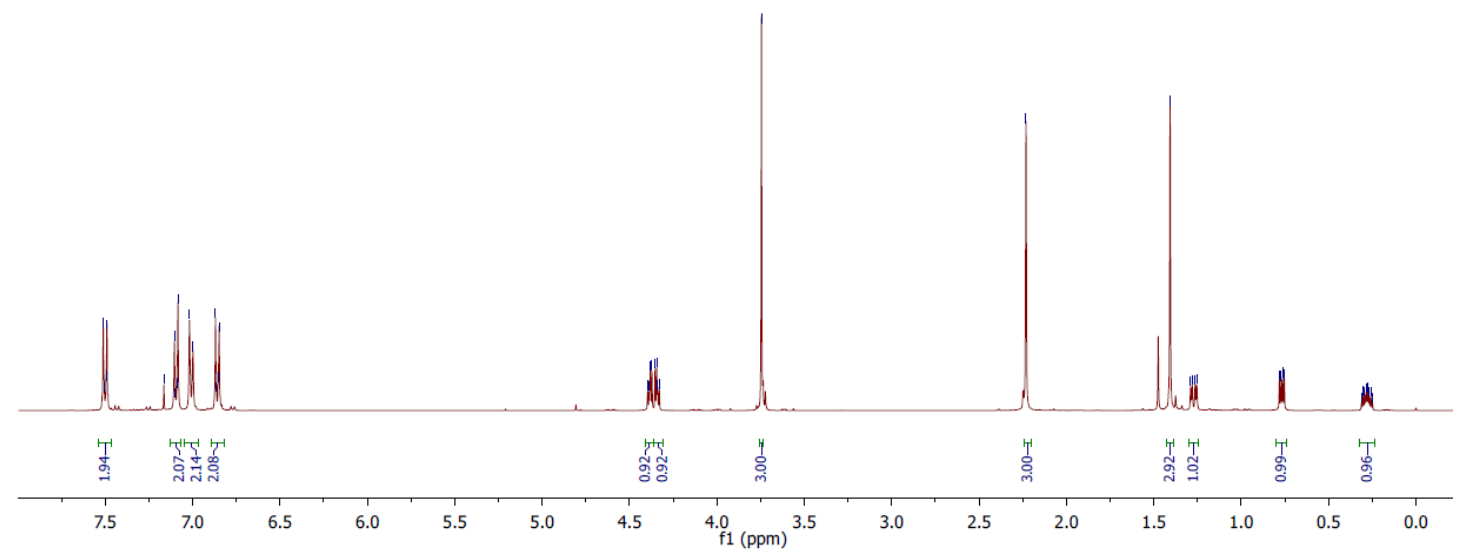

${ }^{13} \mathrm{C}$ NMR (100 MHz, $\left.\mathrm{CDCl}_{3}\right)$

Dian400.3047212.fid dlyc04702

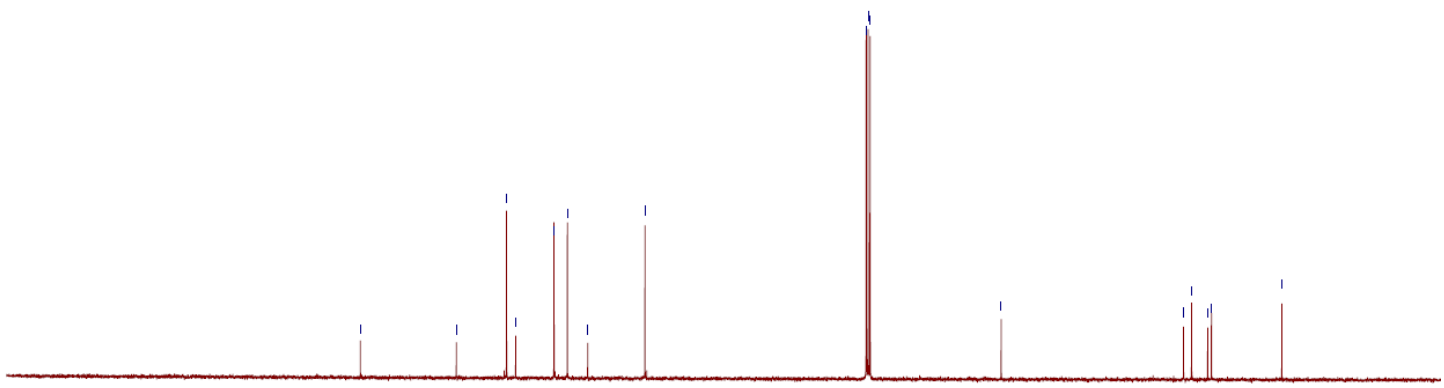

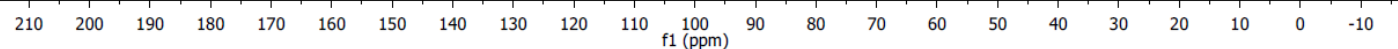


2t

${ }^{1} \mathrm{H}$ NMR (400 MHz, $\mathrm{CDCl}_{3}$ )

dlyC04704p 年整.fide

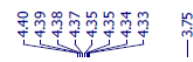

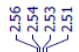

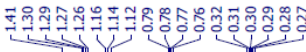

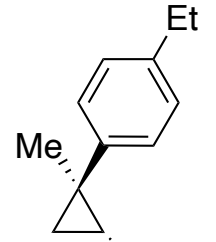

" $\mathrm{SiH}_{2} \mathrm{C}_{6} \mathrm{H}_{4} \mathrm{OMep}$

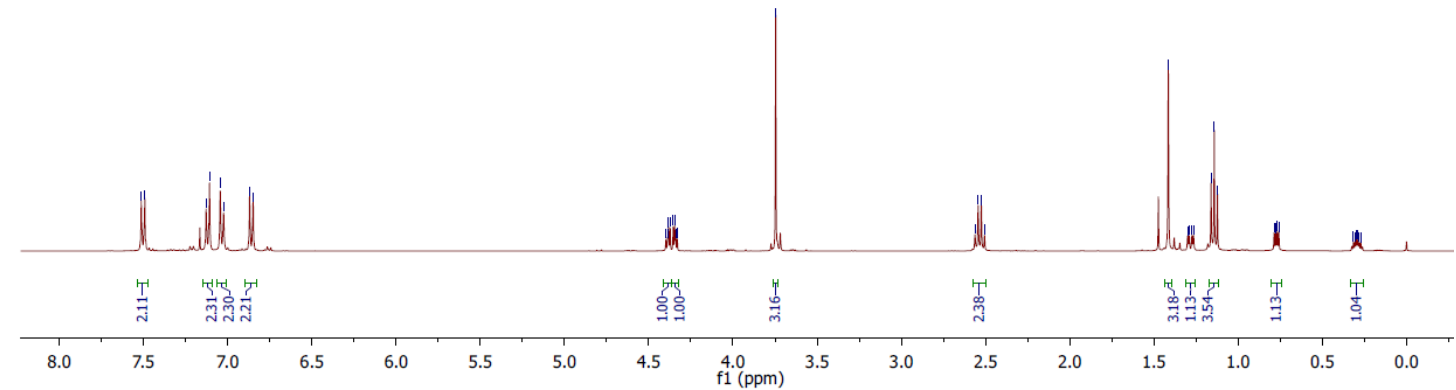

${ }^{13} \mathrm{C}$ NMR (100 MHz, $\left.\mathrm{CDCl}_{3}\right)$

Dian400.30474112.fid dlyc04704p

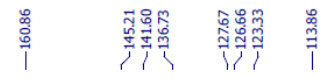

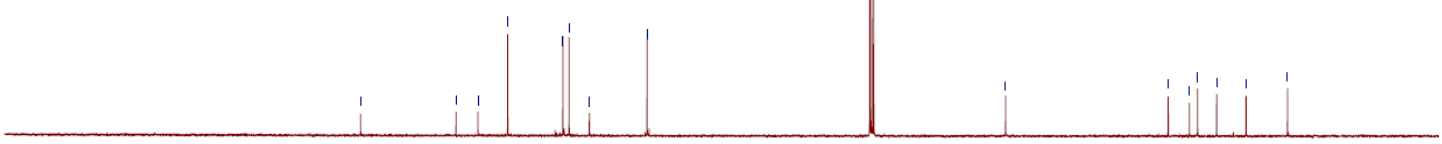

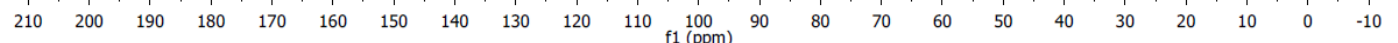


2u

${ }^{1} \mathrm{H}$ NMR (400 MHz, $\mathrm{CDCl}_{3}$ )

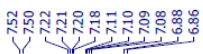

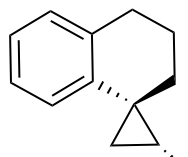

$" \mathrm{SiH}_{2} \mathrm{C}_{6} \mathrm{H}_{4} \mathrm{OMe}-4$

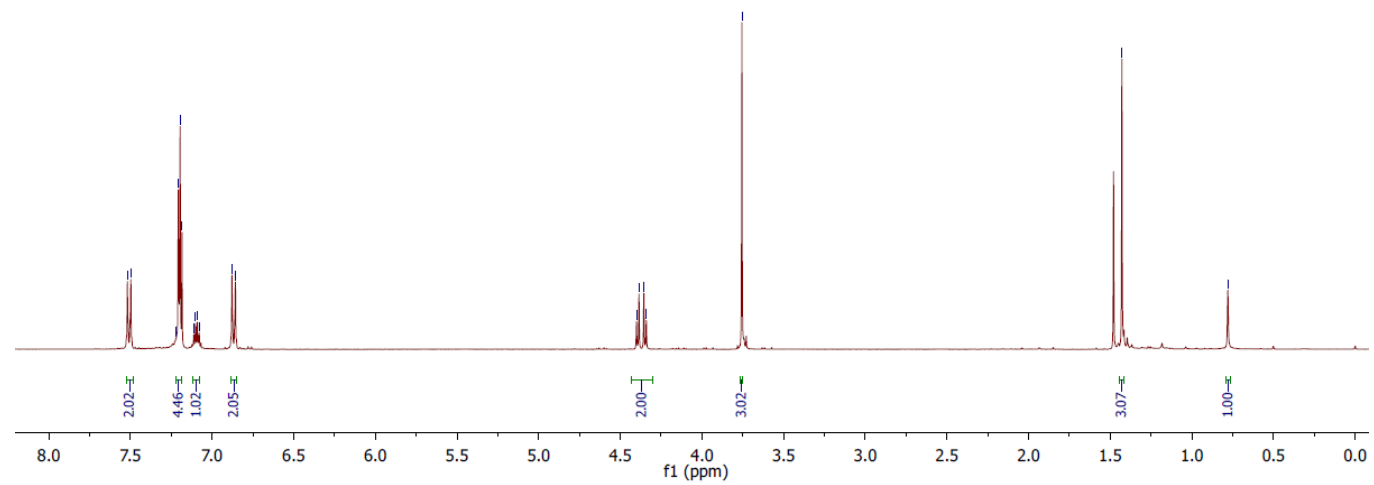

${ }^{13} \mathrm{C}$ NMR (100 MHz, $\left.\mathrm{CDCl}_{3}\right)$

Dian400.30475213.fid

dlyC04705p2

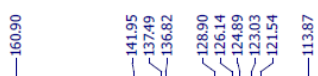

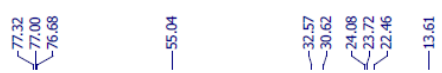

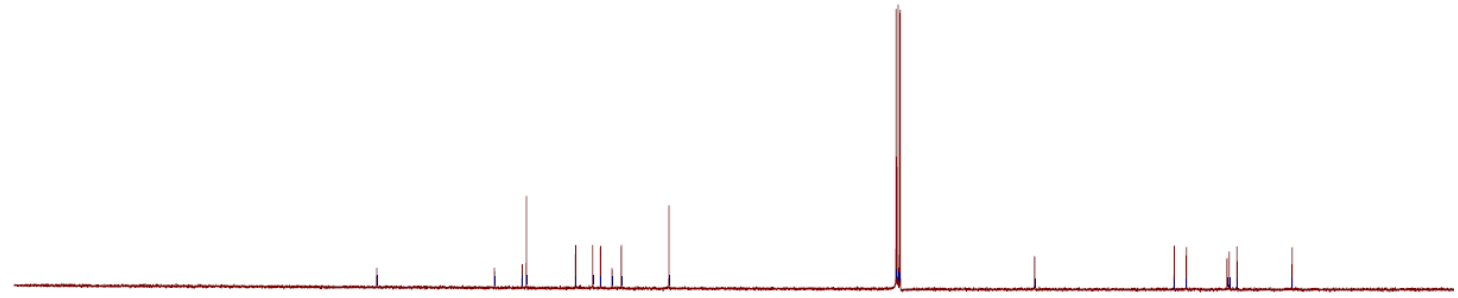

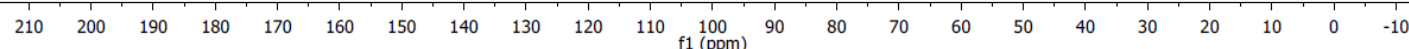


2v

${ }^{1} \mathrm{H}$ NMR (400 MHz, $\left.\mathrm{CDCl}_{3}\right)$
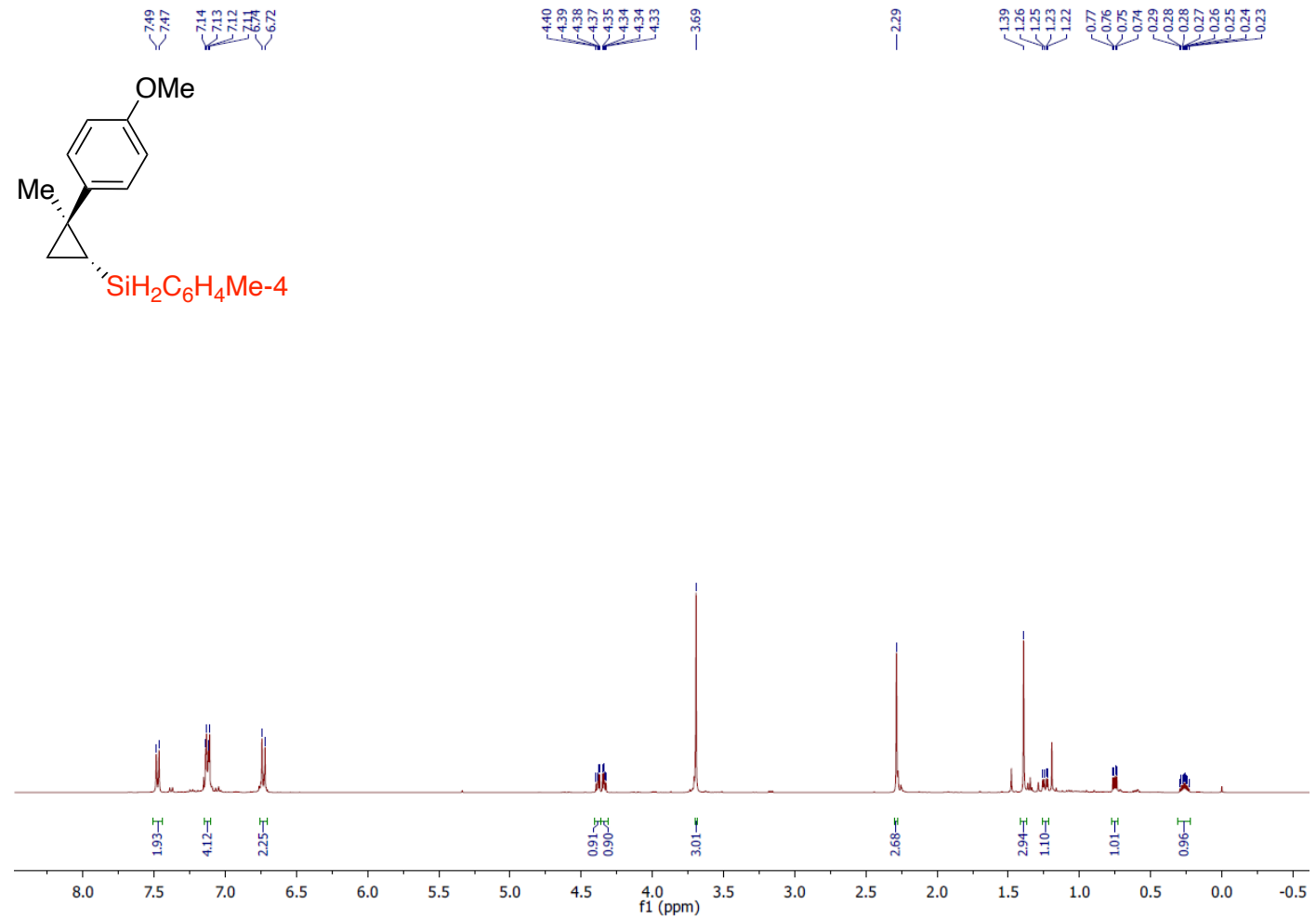

${ }^{13} \mathrm{C}$ NMR (100 MHz, $\left.\mathrm{CDCl}_{3}\right)$

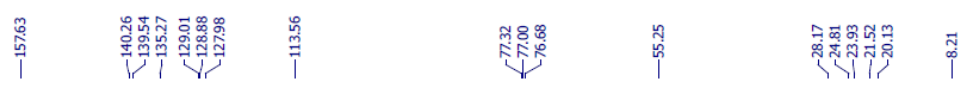

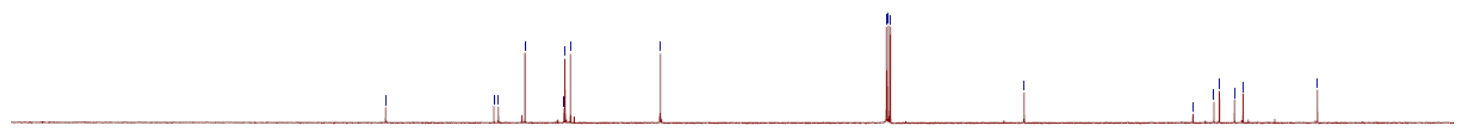

$\begin{array}{llllllllllllllllllllllll}210 & 200 & 190 & 180 & 170 & 160 & 150 & 140 & 130 & 120 & 110 & 100 & 100 & 10 & 70 & 60 & 50 & 40 & 30 & 20 & 10 & 0 & -10\end{array}$ 
2w

${ }^{1} \mathrm{H}$ NMR (400 MHz, $\mathrm{CDCl}_{3}$ )

Dian400.304783111.fid

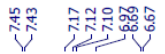

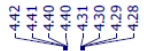

融

1

"SiH $\mathrm{C}_{6} \mathrm{H}_{4} \mathrm{Me}-4$

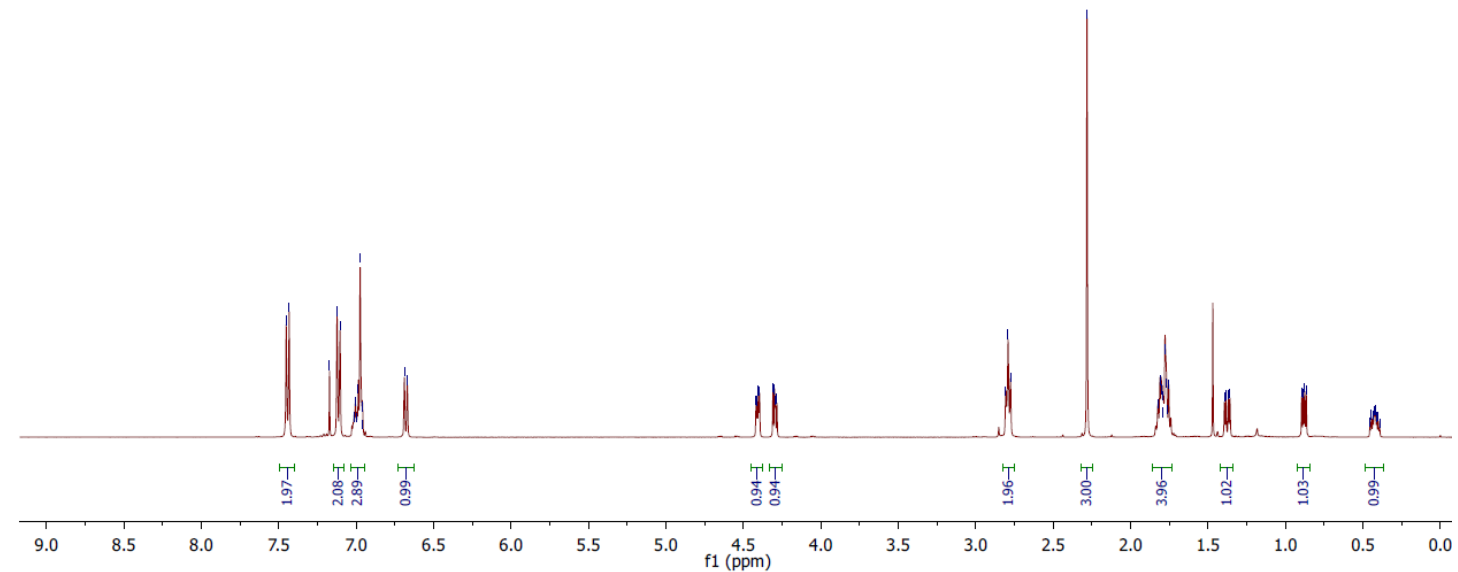

${ }^{13} \mathrm{C}$ NMR (100 MHz, $\left.\mathrm{CDCl}_{3}\right)$

Dian400.304783112.fid dlyC04708p3

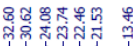

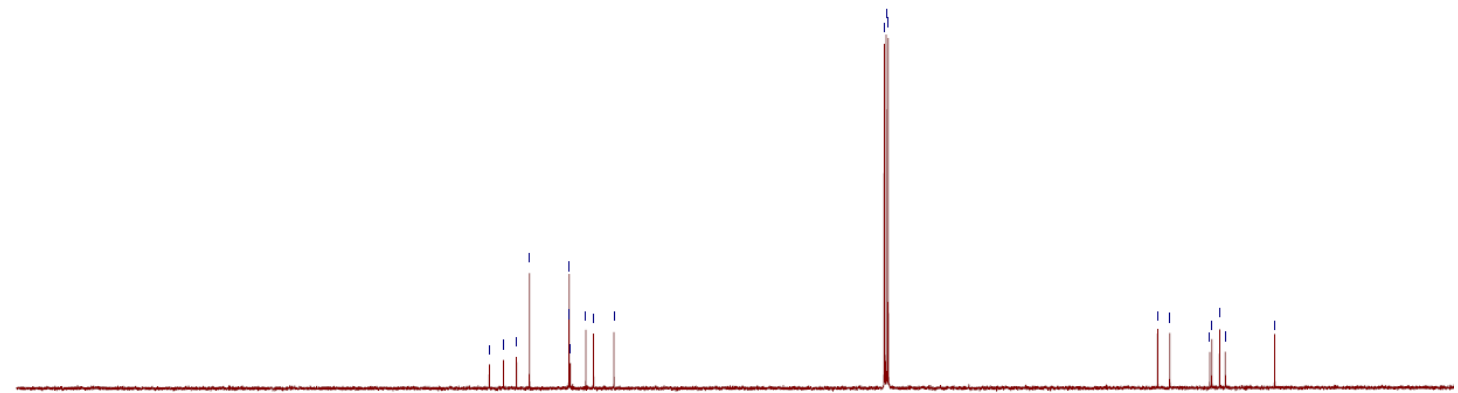

$\begin{array}{lllllllllllllllllllllll}210 & 200 & 190 & 180 & 170 & 160 & 150 & 140 & 130 & 120 & 110 & \begin{array}{c}100 \\ \mathrm{f} 1\end{array} & 90 & 80 & 70 & 60 & 50 & 40 & 30 & 20 & 10 & 0 & -10\end{array}$ 